TM-71-1013-5

\title{
TECHNICAL
} MEMORANDUM

\section{SUPERSONIC FLOW OVER CONVEX \\ AND CONCAVE SHAPES WITH RADIATION \\ AND ABLATION EFFECTS}

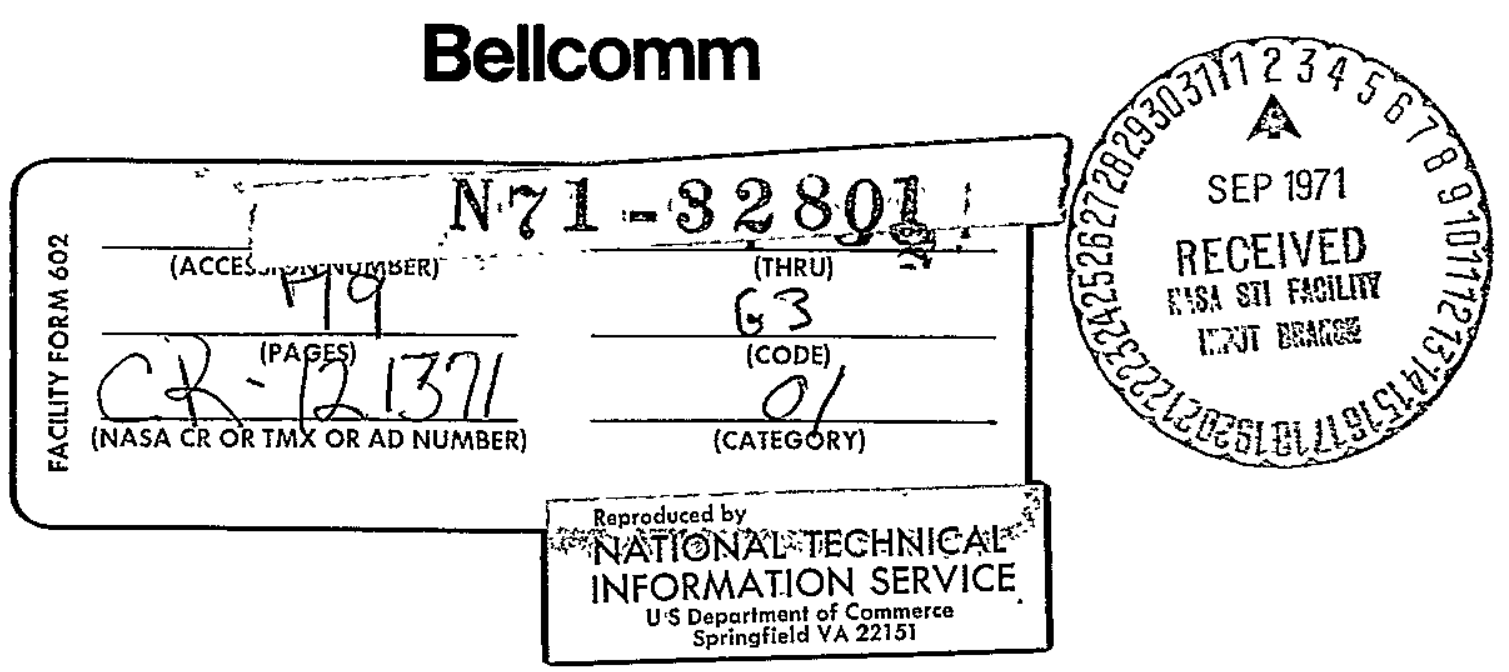




\section{COVER SHEET FOR TECHNICAL MEMORANDUM}

\section{title- Supersonic Flow Over Convex and Concave.Shapes with Radiation and $\mathrm{Ablation}$ Effects}

FILING CASE NO(S)- 105-9

$\begin{array}{ll}\text { FILING SUBJECT(S) } & \text { Atmospheric Entry } \\ \text { (ASSIGNEDBYAUTHOR(s))- } & \text { Flowfields } \\ & \text { Radiating Flows }\end{array}$

\author{
TM- 7I-1013-5 \\ DATE-August 2, 1971 \\ AUTHOR(s)-I. O. Bohachevsky \\ R. N. Kostoff
}

ABSTRACT

A first-order accurate numerical method of unsteady adjustment is employed to calculate inviscid flowfields about convex and concave shapes. Two geometries are used: 1) conical forebodies with spherical afterbodies whose cone half-angles range from $50^{\circ}$ to $135^{\circ}$ and 2) thin-walled cylinders with cylindrical cavities of different depth facing the oncoming stream.

Computations with radiative heat transfer effects are carried out for two cases, namely, flow over a $50^{\circ}$ and an $80^{\circ}$ half-angle cone. A grey gas model is assumed; unlike previous studies, no restriction is placed on the gas optical thickness nor is a slab approximation made. Results show a significant departure from the slab approximation near surface discontinuities and large radiative energy losses near cold surfaces.

For a $50^{\circ}$ half-angle cone a calculation is performed with massive gas injection at the cone surface. Results show that the bow shock detaches from the surface and the shock layer divides into an inner layer containing the injected gas and an outer layer without injectant.

Flows over concave shapes, i.e., the $120^{\circ}$ and $135^{\circ}$ cones and the two cylinders utilized, relax to their steadystate configurations in a damped oscillatory manner. Higherorder accurate computations are necessary to resolve the nature of these oscillations. 


\section{Bellcomm}

date August 2, 1971

to Distribution

from: I. O. Bohachevsky, R. N. Kostoff

subject Supersonic Flow Over Convex and Concave Shapes with Radiation and Ablation Effects Case 105-9
955 L'Enfant Plaza North, S.W. Washington, D. C. 20024

$T M-7 I-1013-5$

\section{TECHNICAL MEMORANDUM}

\section{INTRODUCTION}

Knowledge of the flowfield about a spacecraft entering an atmosphere is important in vehicle design and in the planning of mission profiles. For example, the flowfield determines the deperidence of aerodynamic loads and heat transfer rates (both convective and radiative) on entry trajectory; through specification of the charged particle concentration within the flowfield it delineates that portion of the entry path where communication blackout occurs. Also, knowledge of the flow pattern assists experimenters in, the selection of the most favorable locations on the vehicle surface for placement of their instruments.

During past years, systems studies on planetary entry vehicles were performed at Bellcomm [1] with the purpose of selecting desirable vehicle shapes for a wide variety of missions. At the time of these studies, no general techniques were available that would show the variations of flowfields with vehicle shape throughout the range of attached and detached bow shock configurations. To remedy this deficiency, it was decided to develop a method with the above capability [2] .

Extensive and successful studies of flowfields associated with atmospheric entry vehicles have been performed in the past; the literature pertaining to these investigations is too vast to be discussed here. It is known that, depending on the nose geometry and the free stream conditions, either of two cases occurs: the shock wave is attached with supersonic flow behind it, or the shock wave is detached with both the subsonic and supersonic flows behind it. 
The above two cases have always been investigated separately; to the best of our knowledge no method for determining the flowfield exists that does not require prior knowledge of whether or not the shock is attached. It is desirable to be able to perform flowfield computations for a portion of the trajectory along which the shock configuration changes from attached to detached. Such change of configuration may be caused by the change in free stream conditions or by large ablation rates and excessive blunting of the tip due to erosion.

In addition to these considerations, recent planning of future space missions envisages extensive use of mass injection from the forebody of the vehicle into the flowfield. This may be either for cooling [3] or, through the application of retrorockets, for assisting in the aerodynamic braking during atmospheric entry [4]. Effective exploitation of these devices and related ideas will depend on understanding of the flowfields and the ability to determine these for all entry conditions.

For the above and similar reasons a method is desirable for easy determination of flowfields during reentry without previous knowledge of the nature of the shock configuration.

A numerical method for computation of flowfields developed for engineering applications has to be both relatively accurate and conceptually simple. It should also possess sufficient generality to be applicable to flows with large surface mass injection and radiative heat transfer as well as to asymmetric flows corresponding to vehicles at angles of attack. Finally, it should be easy to modify in case the need for an improvement in accuracy and/or resolution becomes evident in applications to problems where accuracy is crucial, e.g., shockon-shock interactions or oscillating shock problems.

The method would depend in part on the family of shapes to be examined. For study purposes we selected a shape consisting of a conical forebody connected to a spherical afterbody; such a shape allows representation of major classes of different flowfields with the variation of a single parameter, the cone half-angle, in the range from $0^{\circ}$ to $180^{\circ}$. This range covers the small angle case of attached shock. (F.ig. IA), the medium angle case of detached steady-state bow shock (Fig. IB), and the large angle case where oscillating. bow shocks may occur (Fig. IC). 
The purpose of the present paper is to describe a method meeting the above discussed requirements and to present some-results obtained with it.

The oscillatory behavior of flows over conical cavities $\left(120^{\circ}\right.$ and $135^{\circ}$ cones) motivates an additional study of flows over concave shapes. The configuration selected for this purpose is that of a thin-walled cylinder with the open end facing the flow.

The present report is divided into six sections: the background of the problem has been discussed above; Section II, Formulation of the Problem, describes in detail the mathematical problem posed; and Section III, Numerical Procedure, presents the finite difference method used in the solution. Section IV, Radiative Effects, describes the routine used to calculate the radiative energy transfer in the flowfield. Section V, Numerical Results, presents the results obtained during the study. Finally, Section VI, Concluding Remarks, gives a summary of findings and indicates follow-on studies which would be of interest.

In addition, Appendix I lists the detailed algebraic expressions used in the numerical computations, and Appendix II lists the routine for computing radiative transfer.

II. FORMULATION OF THE PROBLEM

A. The General Approach

To determine the flowfield about a prescribed body shape it is necessary to solvel the conservation equations of gas dynamics for a given gas model subject to appropriate boundary conditions. When an inviscid flowfield contains the detached shock, the steady-state equations are hyperbolic in the supersonic region and elliptic in the subsonic region leading to a mixed problem for which the solution is exceedingly difficult and complex. The method selected for solving the problem in the present investigation is based on the unsteady approach in which the time dependent form of the conservation equations is solved. The steady-state solution is that which results after a long time, i.e., the large-time asymptote. The 
unsteady form of conservation equations is everywhere hyperbolic; thus the problem has been converted from a mixed hyperbolicelliptic boundary value problem to a hyperbolic initial-boundary value problem which is mathematically more tractable. This approach has been previously applied successfully to aerodynamical problems by several investigators [5] [6] [7] [8] [9].

Reference 10 presents results for a three-dimensional flowfield about a sharp cone with attached shock. The approach used in this study of flows over conical bodies parallels in some aspects the method reported in Ref. II where, however, a satisfactory treatment of the cone vertex is lacking.

\section{B. Coordinate System}

To simplify the application of the boundary conditions, in general it is convenient to employ a coordinate system with coordinate surfaces that coincide with the given boundaries. When computing flows over cones with spherical afterbodies, it is natural to use a spherical polar coordinate system as shown in Fig. 2, where $x$ is the distance from the origin, and $\theta$ and $\phi$ are the two angular coordinates. The cone axis is directed along the line $\theta=0$ and its vertex is located at $r=0 . \cdot \theta=\theta_{C}=$ constant represents the cone surface, while $r=r_{c}=$ constant represents the spherical afterbody surface. For symmetric flows in the absence of radiation, $\phi$ becomes an ignorable coordinate. When radiative effects are introduced in section IV, $\phi$ will be taken into account.

When flows over thin-walled cylinders are examined, it is natural to use a cylindrical coordinate system in which $x$ is the distance along the cylinder axis, $y$ is the radius normal to cylinder axis, and $\phi$ is the azimuthal coordinate. For symmetric flows $\phi$ is again an ignorable coordinate.

\section{Differential Equations}

In spherical polar coordinates with the azimuthal $(\phi)$ variation suppressed, the governing differential equations for the conservation of mass, momentum, and energy are [5] [6]:

$$
\frac{\partial}{\partial t}\left(\rho r^{2} \sin \theta\right)+\frac{\partial}{\partial r}\left(\rho \operatorname{ur} r^{2} \sin \theta\right)+\frac{\partial}{\partial \theta}(\rho v r \sin \theta)=0
$$




$$
\begin{aligned}
& \frac{\partial}{\partial t}\left(\rho u r^{2} \sin \theta\right)+\frac{\partial}{\partial r}\left[\left(\rho u^{2}+p\right) r^{2} \sin \theta\right]+\frac{\partial}{\partial \theta}(\rho u v r \sin \theta) \\
& =\left(2 p+\rho \cdot v^{2}\right) r \sin \theta \\
& \begin{aligned}
\frac{\partial}{\partial t}\left(\rho v r^{2} \sin \theta\right)+ & \frac{\partial}{\partial r}\left(\rho u v r^{2} \sin \theta\right)+\frac{\partial}{\partial \theta}\left[\left(\rho v^{2}+p\right) r \sin \theta\right] \\
& =p r \cos \theta-\rho u v r \sin \theta \\
\frac{\partial}{\partial t}\left[\left(\frac{1}{2} \rho-q^{2}+\rho h\right.\right. & \left.-p) r^{2} \sin \theta\right]+\frac{\partial}{\partial r}\left[\left(\frac{1}{2} q^{2}+h\right) \rho u r^{2} \sin \theta\right] \\
+\frac{\partial}{\partial \theta} & {\left[\left(\frac{1}{2} q^{2}+h\right) \rho v r \sin \theta\right]=0 }
\end{aligned}
\end{aligned}
$$

where $u$ and $v$ are velocity components in $r$ and $\theta$ \%directions, $q^{2}=u^{2}+v^{2}, \rho$ is the density, $p$ is the pressure, $h$ is the enthalpy per unit mass, and $t$ represents time.

The above form of conservation equations was first introduced by Lax for one-dimensional flows [12] $\because$. In that case the conservation variables remain continuous across a shock, whereas the primitive variables, pressure, density, and velocity are discontinuous there. In multi-dimensional flows, where the velocity components are not necessarily normal to the shock, the conservation variables may no longer remain continuous across it. However, as demonstrated in Refs. 5,6 , and 10 , they remain useful for the computation of shocked flows, probably because magnitudes of discontinuities are. decreased in the conservation form.

In cylindrical coordinates the axisymmetric flow is described by the following system of equations [5]:

$$
\begin{gathered}
\frac{\partial}{\partial t}(\rho y)+\frac{\partial}{\partial x}(\rho u y)+\frac{\partial}{\partial y}(\rho v y)=0 \\
\frac{\partial}{\partial t}(\rho u y)+\frac{\partial}{\partial x}\left[\left(\rho u^{2}+p\right) y\right]+\frac{\partial}{\partial y}(\rho u v y)=0
\end{gathered}
$$




$$
\begin{gathered}
\frac{\partial}{\partial t}(\rho v y)+\frac{\partial}{\partial x}(\rho u v y)+\frac{\partial}{\partial y}\left[\left(\rho v^{2}+p\right) y\right]=p \\
\frac{\partial}{\partial t}\left[\left(\frac{1}{2} \rho q^{2}+\rho h-p\right) y\right]+\frac{\partial}{\partial x}\left\{\left[\frac{1}{2} q^{2}+h\right] \rho u y\right. \\
\left.+\frac{\partial}{\partial y}\left[\frac{1}{2} q^{2}+h\right] \rho v y\right\}=0 .
\end{gathered}
$$

The remarks made about Eqs. (1) -(4) apply here also.

Both systems of Eqs. (I) $-(4)$ and (5)-(8) are singular along the axis of symmetry; equations valid along this line are obtained from (1) $-(4)$ and $(5)-(8)$ after division by $\sin \theta$ or $y$ respectively and passage to the limit $\theta \rightarrow \pi$ or $y \rightarrow 0$ [5]. In the evaluation of these limits the symmetry of the field and L'Hospital's rule are used.

The result along the axis $\theta=\pi$ is

$$
\begin{gathered}
\frac{\partial}{\partial t}\left(\rho r^{2}\right)+\frac{\partial}{\partial r}\left(\rho u r^{2}\right)+2 \frac{\partial}{\partial \theta}(\rho v r)=0 \\
\frac{\partial}{\partial t}\left(\rho u r^{2}\right)+\frac{\partial}{\partial r}\left[\left(\rho u^{2}+p\right) r^{2}\right]+2 \frac{\partial}{\partial \theta}(\rho u v r)=2 p r \\
\frac{\partial p}{\partial \theta}=0 \\
\frac{\partial}{\partial t}\left[\left(\frac{1}{2} \rho q^{2}+\rho h-p\right) r^{2}\right]+\frac{\partial}{\partial r}\left[\left(\frac{1}{2} q^{2}+h\right) \rho u r^{2}\right] \\
+2 \frac{\partial}{\partial \theta}\left[\left(\frac{1}{2} q^{2}+h\right) \rho v r\right]=0 .
\end{gathered}
$$

and along the axis $y=0$ it is:

$$
\frac{\partial}{\partial t} \cdot \rho+\frac{\partial}{\partial x}(\rho u)+2 \frac{\partial}{\partial y}(\rho v)=0
$$




$$
\begin{gathered}
\frac{\partial}{\partial t}(\rho u)+\frac{\partial}{\partial x}\left(\rho u^{2}+p\right)+2 \frac{\partial}{\partial y}(\rho u v)=0 \\
\frac{\partial p}{\partial y}=0 \\
\frac{\partial}{\partial t}\left[\left(\frac{1}{2} \rho q^{2}+\rho h-p\right)+\frac{\partial}{\partial x}\left\{\rho u\left[\frac{1}{2} q^{2}+h\right]\right\}\right. \\
+2 \frac{\partial}{\partial y}\left\{\rho v\left[\frac{1}{2} q^{2}+h\right]\right\}=0 .
\end{gathered}
$$

\section{Boundary Conditions}

To complete the formulation, of the problem the differential equations must be supplemented with suitable boundary conditions.

At the solid surfaces of the body, the boundary condition should require only the vanishing of the normal velocity component. At the outer edge of the computational field, upstream of the nose part, the solution. must assume the prescribed free stream values. Downstream. of the nose section, the flow variables should tend to approximately constant values along the individual streamlines. Along the axis, the usual symmetry conditions apply.

The origin, $r=0$, is the singular point of the spherical coordinate system and consequently of systems (1) through (4) and (9) through (12); this singularity differs from the singularity on the axis in that it is no longer possible to obtain here a valid system of equations by any limiting process. At the present time, all that will be required is the regularity of the solution at $r=0$; the way this condition is imposed will be discussed in Sec. III-B. Similar statements apply also to sharp corners on the body.surface; the treatment of these loci will be described later.

For the present problem, in which the steady-state solution is of prime interest, initial conditions could, in principle, be prescribed arbitrarily. A straightforward procedure is to start the body impulsively with the free stream velocity; free stream values are thus prescribed as initial conditions everywhere in the field. Different ways of prescribing the initial conditions will be mentioned later. 


\section{E. Thermodynamic Gas Model}

To form a closed system, the conservation equations must be supplemented with a thermodynamic relation between the state variables density $\rho$, pressure $p$, and enthalpy $h$. For an ideal gas, which is considered here, this relation is:

$$
h=\frac{\gamma}{\gamma-1} \frac{p}{\rho}
$$

In general $\gamma$, the ratio of specific heats, is not a constant but a function of temperature and density. However, as Ref. 13 shows, an "effective" $\gamma$ may be obtained by tabulating the quantity $\frac{h \rho}{p}$ and choosing a constant $\frac{\gamma}{\gamma-1}$ which best approximates these values of $\frac{h p}{p}$. In the present computations $\gamma=1.4$ is used for moderate Mach numbers and $\gamma=1.2$ for hypersonic Mach numbers when the effect of radiation is taken into account.

III. NUMERICAI PROCEDURE

\section{A. Background}

The differencing scheme used in our computations is an adaptation of the one originally proposed by P. D. Lax [12] . It is the simplest technique available [5] [6] [10] and is easy to modify. As Richtmyer and Morton have observed [14], successive repetition of computational steps in Lax's scheme with only minor modifications (the two-step method) results in an equivalent to the second-order accurate Lax-Wendroff scheme [15]. A further increase in accuracy, to third order, is also possible by the addition of a moderately complicated third iteration; the final result becomes equivalent to the scheme devised recently by Rusanov [16].

\section{B. Computational Mesh}

The mesh employed in finite difference computations for conical geometry is shown in Fig. 3. It is similar to the grid used on commonly available polar-coordinate graph paper in which new angular divisions, $\Delta \theta$, are added as the distance from the origin increases so that the linear mesh dimensions are approximately equal in all sections of the grid. Computations in which a varying linear mesh size (constant angular steps) was 
used demonstrated that the maximum time step was governed by the smallest linear mesh dimension, resulting in unequal resolution in different regions of the field. To obtain equal resolution in all parts of the field it is necessary to have the same mesh dimensions throughout. In practice, for increasing $r$, whenever $r \Delta \theta$ doubles the value of $\Delta \theta$ is halved. Thus the product $r \Delta \theta$ is kept approximately equal to the radial increment $\Delta r(\Delta r=1 \mathrm{~cm})$. The arcs along which new angular divisions are added are called internal boundaries.

The mesh used in connection with cylindrical coordinates is shown in Fig. 4; it is rectangular with $\Delta x=\Delta y=1 \mathrm{~cm}$.

C. Finite Difference Equations

On the computational grids shown in Figs. 3 and 4 , the finite difference analogue of the differential form of the conservation equations is constructed in the following manner. Space derivatives in the differential equations are replaced by first central differences and time derivatives by first forward differences. The first-order accurate scheme thus obtained, however, is known to be unconditionally unstable; to stabilize the computations, a second-order dissipative term in the form of a Laplacean of the conservation variable is added [12] [5]. The final algebraic expressions used in numerical calculations are listed in Appendix I.

Clearly, the finite difference scheme described above and presented in Appendix $I$ is explicit; $i . e .$, the values of flow variables at time $t+\Delta t$ are expressed in terms of those at $t$ ime $t$. Therefore, starting with any given initial distribution, the flowfield can be advanced stepwise in time as far as desired.

\section{Boundary Conditions for the Difference Equations}

Because central differences and higher-order dissipative terms are used that necessitate specification of variables at points exterior to the computational mesh when advancing boundary points with time, boundary conditions additional to those stated in Sec. II-D are required. There is no a priori physical basis for their specification; these conditions can be justified only a posteriori. To facilitate further discussion of the boundary conditions, different segments of the boundary are labeled in Figs. 3 and 4 .

At the solid boundaries (OF and FE in Fig. 3) the first derivatives normal to the surface of all flow variables, with the exception of the normal velocity component, are set equal to zero. 
The normal velocity component is extrapolated through zero at the surface; this appears to be a good approximation for flows with small gradients near the wall and it automatically satisfies the normal momentum equation at the surface. For flows with large vorticity, such as those including surface máss injection, the pressure at the exterior point is specified by requiring that with the prescribed value of mass addition at the surface, the normal momentum equation at the surface i.s satisfied.

The corner point $F$ is treated as a triple valued point. Values at $F$ are advanced as though $F$ were not on the boundary but in the flowfield. This results in a slight rounding of the corner. When the boundary points adjacent to $F$ are advanced, they each consider $F$ as a point on their respective boundary. Treating the singularity in this fashion locates the sonic line at the corner and helps to..control the local expansion gradients; it constitutes an improvement over the handing of this point in Refs. 5 and 6 .

The auxiliary points at the outer upstream boundary (arc $A B$ and line $B C$ ) are assigned free stream values throughout the computation. At the downstream end of the mesh, the flow variables are extrapolated linearly to the exterior points beyond arc $\mathrm{CD}$ and line $\mathrm{DE}$.

At the internal boundaries, (arcs across which the size of $\Delta \theta$ changes) the values between mesh points on $r-\Delta r$ are obtained from a linear interpolation between the values at the two adjacent points on that arc.

On the axis of symmetry of the flow, the reflection condition is used [5].

The cone.vertex, point 0 , is a singular point in the field and an improper treatment of it will greatly distort the flowfield [11]. In the present form of the difference equations the coeficicients of all variables evaluated at the vertex vanish and thus the solution is independent of the manner in which values at the vertex are specified. For purposes of the presentation of results which include the vertex, this point is treated as a multi-valued one with values extrapolated along radial lines to the apex.

For computations in cylinarical coordinates, the boundary conditions are specified ana.ogously to the above procedure. At the solid boundaries EF, ED, and FG in Fig. 4 the same conditions are prescribed as on the surface of the cone. 
The end of the flange, point $\mathrm{E}$ in Fig. 4, is treated as a triple valued point like the corner of the cone. At the points $\mathrm{F}$ and $\mathrm{G}$ inside the cylindrical cavity, stagnation conditions are prescribed. Along the axis of symmetry, AG, and the other boundaries of the mesh, $A B, B C$, and $C D$, the same conditions are used as on the corresponding segments in the conical geometry.

The initial state of the field may be specified arbitrarily; it is simplest to begin the computations from the prescription of free stream values everywhere, as stated in Sec. I-D. For many purposes, however, it is convenient to store the results of one computation on a magnetic tape and use them later as initial conditions for new calculations. In this way considerable amounts of computer time are saved when solving a series of problems or investigating parametric deperidence.

After the field is initialized the boundary conditions are applied to initialize the region exterior to the field. The finite difference equations are then used to advance the variables from their values at $t=0$ to values at $t=\Delta t$ and the process is repeated until the desired result is obtained.

To assure stability of the computation the time increment $\Delta t$ used must be that which is given approximately by the Courant-Friedrichs-Lewy condition [5] [17]; it requires that numerical propagation speed be not less than the fastest physical propagation speed. In practice the maximum $\Delta t$ which can be used consistent with stable computations is determined by experimentation to be within approximately $20 \%$ of the value given by the $\mathrm{C}-\mathrm{F}-\mathrm{I}$ condition. It is desirable to use the largest possible $\Delta t$ in order to minimize the smearing of the shocks.

\section{RADIATION EFFECTS}

For high velocity flight, e.g., superorbital entry conditions, a satisfactory description of the flowfield should include the effects of radiative heat transfer. Two major difficulties arise in the treatment of this phenomenon: the variation of the absorption and emission coefficients with frequency and the three-dimensional nature of the radiation field.

In the present paper we concentrate on the geometrical aspect of the problem for the flow over a cone; i.e., the determination of radiative contribution from $4 \pi$ steradians without any restrictive assumptions on the symmetry properties of the 
radiating field. This end is accomplished conveniently by introducing, for the purpose of integration, a local spherical coordinatè system at each mesh point of the flowfield. Since the thermodynamic properties of the medium are referenced in a coordinate system centered at the vertex of the cone, the evaluation of radiation integrals requires application of transformation formulae between two spherical coordinate systems.

Previous calculations of radiation phenomena were concerned mainly with the construction of band absorption models for the treatment of frequency dependence. The literature pertaining to this topic is too rich to be discussed here; an adequate review is given in a recent survey article by Anderson [18] . In these investigations of spectral effects the assumption of slab symmetry has always been made. This simplification, however, does not appear to be justified for a sharp cone because the tangent plane is not a good approximation to the cone surface near the vertex.

For flows in which energy transport by radiation is significant, i.e., when free-stream Mach number $\mathrm{M}_{\mathrm{O}}>230$, the equation for conservation of total energy, Eq. (4), must be modified by adding to the right-hand side a term, $Q$, that represents the heat gained per unit volume from radiation transport. It is convenient and therefore customary to express this energy gain as the difference between the absorption and emission at the given point.

To describe the numerical computation of three-dimensional radiative effects and to indicate the assumptions made for that purpose, we first sketch the derivation of the analytic expression for $Q$. Towards this end we introduce at each meshpoint of the flowfield a spherical coordinate system, as shown in Fig. 2, where $\psi$ denotes the angle measured from the extension of the radius $r$, $\lambda$ denotes the azimuth angle around $r$, and $R$ the distance along the ray $(\psi, \lambda)$. Then, for each frequency $v$ we obtain at the point
$\left(\theta_{i}, r_{j}\right):$

$$
Q_{i, j}=\dot{j} s_{4 \pi}(A-E) d \omega
$$

where $A$ is the absorption, $E$ the emission, and $\mathrm{d} \omega$ is the area element on the unit sphere, $\mathrm{d} \omega=\sin \psi \mathrm{d} \psi \mathrm{d} \lambda$; the symbol $4 \pi$ indicates that the integration extends over the unit sphere. 
If we now assume that the state of quasi-equilibrium prevails [19], then each element of the gas emits as if it-were in equilibrium with its surroundings and the emission is given by:

$$
E_{i, j}=(\varepsilon B)_{i, j}
$$

where $\varepsilon$ is the emissivity and $B$ is the Planck's function:

$$
B=\frac{2 h \nu^{3}}{c^{2}} \frac{1}{\exp \frac{h \nu}{R T}-1}
$$

in which $\mathrm{h}$ is the Planck constant, $v$ is the frequency of radiation $c$ is the speed of light, $k$ is the Boltzman constant, and $T$ is the local temperature.

The assumption of quasi-equilibrium, however, does not imply that absorption equals emission because, unlike emission, absorption is not a local property but depends on the emission from all regions of the medium, some of which are at temperatures drastically different from $T_{i, j}[19]$. Therefore, (when scattering is neglected) we have for each ray $(\psi, \lambda)$ :

$$
A_{i, j}=\alpha_{i, j}\left[\int_{0}^{R} s B e^{-\tau} d R+I_{s} e^{-\tau} s\right]
$$

where $\alpha$ is the local absorptivity, $I_{\mathbf{S}}$ is the intensity of the surface radiation, and $\tau$ is the optical thickness,

$$
\tau=\int_{0}^{R} \alpha \mathrm{dR}
$$

In Eq. (21) the integration extends from the point $\left(\theta_{i}, x_{j}\right)(R=0)$ to the solid surface $R=R_{S}$ if the ray intercepts one; if not, 
then the integration extends to $\infty$ and the surface contribution, $I_{s} e^{-\tau} s$, is omitted. The range of directions $(\psi, \lambda)$ for which the rays intercept the solid surface of the cone is specified explicitly in Appendix II.

Combining the above considerations we obtain:

$Q_{i, j}=\iint_{4 \pi}\left\{\alpha_{i, j}\left[\int_{0}^{R_{S}} \alpha B e^{-\tau} d R+I_{s} e^{-\tau} s\right]-(\varepsilon B)_{i, j}\right\} \sin \psi d \psi d \lambda$.

Eq. (23) holds for each frequency $v$ and must be integrated with respect to $v$ to represent the total radiative energy contribution. This integration process is usually circumvented, as noted above, by the construction of band absorption models. The simplest of these, of course, is a grey gas model in which $\alpha$ and $\varepsilon$ are independent of $v$; then the frequency integration can be carried out explicitly,

$$
\int_{0}^{\infty} B d v=\frac{\sigma}{\pi} T^{4}
$$

( $\sigma$ is the Stefan-Boltzman constant) and the expression for $Q_{i, j}$ simplifies considerably. With the additional assumption of isotropic emission it becomes:

$$
\begin{aligned}
Q_{i, j}=\frac{\sigma}{\pi}\left[\alpha_{i, j} \iint_{4 \pi}\right. & \left(\int_{0}^{R_{S}} \alpha T^{4} e^{-\tau} d R+\varepsilon s^{T} s^{4} e^{-\tau} s\right) \sin \psi d \psi d \lambda \\
& \left.-4 \pi\left(\varepsilon \dot{T}^{4}\right)_{i, j}\right]
\end{aligned}
$$

where the subscript s everywhere above denotes, surface values.

As stated previously, we do not pursue this line of investigation any further but instead indicate now a method for 
convenient numerical evaluation of expression (23) or (25) in the case of a given configuration of flow over a cone.

Towards this end, we proceed as follows. At each point $\left(\theta_{i}, r_{j}\right)$ we select a set of pairs $\left(\psi_{n}, \lambda_{m}\right)$ and check each ray $\left(\psi_{n}, \bar{\lambda}_{m}\right)$ for the intersection with the solid surface of the cone; if the ray intersects the cone we compute the distance $R_{S}$ at which that happens and divide it into an integer number of steps dR. If the ray does not hit the cone surface we use integration steps that are large when the ray traverses low temperature regions and small when the ray enters the hot shock layer where the radiative contribution is large and may change rapidly. The transition is arbitrarily set to occur when the temperature exceeds twice the ambient free stream value. We step off along the ray with intervals of length dR until either the cone surface or the end of the mesh is reached and determine the $\theta, r$, and $\phi$ coordinates at the endpoint of each interval. After elimination of the ignorable coordinate $\phi$ we determine from these coordinates, by a double interpolation procedure, the thermodynamic state of the medium at each point on the ray. With this information it is easy to sum over the necessary dR intervals and obtain the optical thickness $\tau$, calculate the corresponding absorption, and sum again (adding the surface term when necessary) to obtain the contribution from the entire ray. A subsequent double summation over $n$ and $m$, with appropriate differentials $d \psi$ and $d \lambda$, that covers the unit sphere completes the computation of radiative energy contribution in each frequency band. By combining the amounts of energy added in each considered frequency range, we obtain the total energy delivered by the radiation transport.

The above described calculational procedure is very well suited for automatic digital computation. Appendix II Iists the necessary expressions and the detailed list of steps required to carry out the computation of radiative energy contribution. The length of actual computations will be discussed in the next section together with concrete-examples.

V. NUMERICAL RESULTS

The finite difference method described above was used to compute flowfields for $50^{\circ}, 80^{\circ}, 90^{\circ}, 120^{\circ}$, and $135^{\circ}$ halfangle cones and thin-walled hollow cylinders at various free stream Mach numbers, $M_{0}$. In the present section we will describe these results in some otetil and present, when possible, comparisons with other calculations or experimental data. The cgs system of units is used throughout. 
It is convenient to present the results in three groups: first we discuss flows over cones with attached and. detached shocks, then we present the three-dimensional radiation effects for some of these flows, and finally we describe flows over conical and cylindrical cavities.

A. Flows Over $50^{\circ}, 80^{\circ}$, and $90^{\circ}$ Half-Angle Cones

1. $50^{\circ}$ Half-Angle Cone

For this geometry the computations were performed at $M_{0}=5$ with the free stream static conditions given by

$\rho_{\circ}=.184 \times 10^{-4} \frac{\mathrm{gram}}{\mathrm{cm}^{3}} \quad$ and $\quad \mathrm{p}_{\circ}=.121 \times 10^{5} \frac{\mathrm{dyne}}{\mathrm{cm}^{2}}$

which correspond to an altitude of approximately $35 \mathrm{~km}$ in the earth's atmosphere. These static conditions will be used in all computations except those including radiation effects. Two cases were considered: a) solid surface (without mass injection), and b) massive gas injection into the shock layer.

\section{a. No Gas Injection}

The steady-state shock positions for a finite length cone without mass injection obtained from our results as well as for an infinite length cone obtained from Ref. 20 are presented in Fig. 5. In the region of conical flow $(r<60 \Delta r$, cone surface extends to $65 \Delta r$ ) the shock angles differ by about two degrees. This may be caused by an effective bluntness of the body which forces the shock to move further out than its ideal sharp cone position. The bluntness results from the combination of "artificial viscosity" with large angular gradients near the vertex. In previous computations, when uniform angular increments throughout the field were used and excellent angular resolution near the vertex attained, the shock angle computed with our method for the finite cone coincided with that tabulated for the infinite cone to within a fraction of a degree. Unfortunately, as mentioned in Sec. III-B, such non-uniform mesh required inappropriately small time increments which severely distorted results away from the vertex and the technique had to be abandoned.

As Fig. 5 shows, the region of conical flow terminates past about $r=60 \Delta r$ where the influence of the corner is felt. This means that the corner affects the flow in the upstream direction, which should not happen in supersonic flow. The effect is caused by the small dissipative terms in the equations 
that allow upstream propagation of the signals. Theoretically, the shock should bend towards the body at the point where the left-running characteristic emanating from the corner intersects the shock. An approximate calculation, assuming this characteristic to be a straight line, gives the point of intersection at $r$ \& $80 \Delta r$ (see Fig. 5).

In order to illustrate how the present method distinguishes between the attached and detached shock configuration, we have prepared Fig. 6. The two curves in that figure represent the pressure variation along the axis-surface streamine for the $50^{\circ}$ and $90^{\circ}$ half-angle cones. We see that for the attached shock configuration $\left(50^{\circ}\right.$ cone) the pressure remains at its free stream value along the axis to within a few mesh points of the vertex and then is perturbed slightly. Across the vertex the pressure jumps to almost $90 \%$ of its correct value behind the attached shock and continues to approach the correct value some distance away from the vertex. In view of the fact that, as Fig. 3 shows, there is only one angular step between the axis and the cone surface at the vertex and for some distance away from the vertex there are no mesh points between the shock and cone surface, such performance of the numerical scheme is quite good. A more elaborate structure of the mesh near the vertex would improve the accuracy and resolution considerably.

one possible configuration would consist of a somewhat finer angular mesh near the vertex combined with the employment of different time increments, $\Delta t$, in regions of different $\Delta \theta$. Then each time advance will be followed by an appropriate interpolation (possibly extrapolation in some regions) procedure set up to adjust all parts of the field to the same time level prior to next advance.

The $90^{\circ}$ half-angle cone results included in this figure will be discussed later.

The monotone approach of the flowfield to its steadystate is shown in Fig. 7. Presented here are the time histories of pressure and density on the cone surface one mesh point away from the vertex; the values are normalized to the ones attained at $t=600 \Delta t$. We see that an essentially steady-state is achieved after about 300 time steps; this number and the monotone nature of the approach will be contrasted later to the oscillatory behavior which occurs for cone angles in excess of $90^{\circ}$.

\section{b. Massive Gas Injection}

The boundary conditions for the computation of mass injection effects presented in Figs. 5 and 8 were specified as follows: 
a. Mass flux injected normal to the conical forebody was set equal to .4 of the free stream mass flux.

b. Tangential velocity at the boundary was set equal to zero.

c. Normal velocity at the exterior point was obtained by extrapolation from the flowfield with a fraction $\alpha$ of the derivative at the boundary. Numerical experimentation showed that $\alpha=.4$ gave the most stable results.

d. Density and tangential velocity at the exterior point were specified with the reflection condition as in the no injection case.

e. Pressure at the exterior point was obtained from the normal momentum equation at the boundary with the above values of normal flux and tangential velocity.

The above prescription of the pressure is less restrictive than the reflection condition and appears more appropriate for large vorticity flows. It would be desir$\mathrm{able}$ to specify also other variables at the exterior point with the aid of the remaining conservation equations; this direction warrants further exploration.

Included in Fig. 5 are the steady-state shock shape for the case of large mass injection as well as the sonic line and an estimate for the position of the shear surface. We observe that now the shock is detached; from previous studies we know that the shock layer is divided into inviscid outer and inner layers separated by a viscous shear surface. The oncoming stream effectively sees the blunt shape defined by the thin shear surface.

The position of the shear surface is obtained from pressure profiles, one of which is shown in Fig. 8. We see from this figure that pressure profiles representing the large mass injection case exhibit a shallow minimum between the shock and cone surface; the position of the shear layer is estimated as the locus of points inside the shock layer with maximum pressure gradient.

This estimate is suggested by the finding of Wallace and Kemp [21] that, in cases when an analytic solution is possible, matching of pressure on both sides of the 
contact surface leàves a discontinuity in pressure gradient. The correctness of the above interpretation is confirmed by an examination of the direction field of velocity vectors which are tangent to the shear surface.

\section{2. $80^{\circ}$ Half-Angle Cone}

For this geometry flowfields without radiation effects were computed at $\mathrm{M}_{0}=4.63, \mathrm{M}_{\mathrm{O}}=20$, and $\mathrm{M}_{\mathrm{O}}=47.2$. The lowest Mach number was selected for the purpose of comparison with experimental results obtained at Iangley Research Center [22].

The steady-state shock positions and sonic lines for $\mathrm{M}_{\mathrm{O}}=4.63$ and $\mathrm{M}_{\mathrm{O}}=20$ flows, together with experimental points of Campbell and Howell [22], are shown in Fig. 9. Agreement between the numerical computations and experimental results is excellent throughout the shock layer because of the large number of grid points between the shock and the body surface that allows the gradients in this region to be satisfactorily resolved. In the attached shock case, few grid points exist in the shock layer near the vertex and consequently the gradients cannot be'resolved as well as in the present case. Because of the higher compression in the shock layer, the shock at $M_{0}=20$ is located significantly closer to the body than at $\mathrm{M}_{0}=4.63$. For both Mach numbers, the sonic line is a smooth curve emanating from the corner and intersecting the shock at an acute angle. Thus, the present treatment of the corner as a triple-valued point improves on previous corner treatments as a single-valued point [5] [6] where the sonic line was found to intersect the body about five mesh points behind the corner. As predicted in Ref. 23, the intersection of the sonic line with the shock moves closer to the axis as the Mach number is increased. The shock inclinations-at the point of intersection agree well with the values of critical angles obtained from the NACA chart [24]:

Shock shapes presented in Fig. 9 are constructed from pressure profiles plotted along spherical surfaces at varying distances from the vertex; the shock is located at the points of maximum gradient. The time history of one representative pressure profile along a surface of radius $r=36 \Delta r$ for $M_{0}=20$ is shown in Fig. 10. All profiles show the pressure continuously increasing from the shock to the body, as they should. 
Fig. 11 represents the variation of pressure along the axis and along the cone surface. As expected, variation of pressure within the shock layer shown on this graph is very small because of the low Mach numbers in the subsonic region. Computed values of pressure along the surface agree to within one percent of the measured values reported in Ref. 22; however, values along the axis within the shock layer are a few percent lower than the pressures obtained from one-dimensional calculations for normal shocks as well as the measured values at the axis-body intersection. As indicated in Sec. II-C, the finitedifference expressions on the axis are different from those used in the rest of the field and we feel that this discrepancy is due to an inadequate numerical representation of the differential equations. The pertinent expressions for the axis should be re-examined.

Steady-state profiles of density along spherical surfaces whose radij are greater than or equal to that of the spherical afterbody are presented in Fig. 12. The profiles for $\mathrm{M}_{\mathrm{O}}=20$ are shown along surfaces of radius (normalized to the spherical afterbody radius) $1.00,1.08$, and 1.13. Of particular interest is the ability of the numerical scheme to follow the large expansion gradient along the rear surface past the corner without producing negative densities. (See Ref. 25 for a report of the appearance of computed negative densities in the corner expansion region.) Away from the rear surface, density gradients decrease as the radius of the surface over which they are presented increases.

The time history of pressure and density near the vertex of the cone for a $M_{0}=4.63$ flow is shown in Fig. 13 . Up to 500 iterations the approach to steady-state is rapid; then slight oscillations occur, after which a more gradual asymptotic path is followed. For the pressure and density to be within .5\% of their steady-state values about 1,000 iterations are necessary. For higher Mach numbers the approach to steady-state is more rapid because the shock layer is thinner. A more detailed discussion of the time dependence of solution will be given later in connection with flows over concave shapes.

Presently we will examine the dependence of the flowfield on the ratio of specific heats $\gamma$. In Figs. 14 and 15 are shown density and pressure for two different values of this ratio, $\gamma=1.2$ and $\gamma=1.4$, computed with $M_{0}=47.2$. We see that a reduction in $\gamma$ is accompanied by a large density increase but 
that the pressure remains essentially unaffected. As a consequence of this the shock layer thickness and the temperature within the shock layer are reduced significantly. Thus, $\gamma$ has a strong effect on the radiative heat transfer.

\section{3. $90^{\circ}$ Half-Angle Cone}

For this geometry, which corresponds to a flat face, computations were performed at $\mathrm{M}_{0}=4.63$ and $\mathrm{M}_{0}=20$.

The steady-state shock positions for both of the above cases are shown in Fig. 16 together with experimental points obtained at Irangley Research Center [22] for $\mathrm{M}_{0}=4.63$ flow and points determined by the method of Integral Relations. Agreement between both sets of numerical results is very good and agreement between numerical and experimental results is good. For the $90^{\circ}$ cone the shock stand-off distance along the axis is about $40 \%$ larger than that for the $80^{\circ}$ cone at both Mach numbers. However, the shortest distance from the corner of the body to the shock remains the same for both cones and varies only with the Mach number. In fact, results reported in Ref. 22 indicate that this distance is the same for $60^{\circ}, 70^{\circ}, 80^{\circ}$, and $90^{\circ}$ cones. This should not be too surprising, for if the minimum corner-shock distance is taken as the analogue to the throat of a nozzle where sonic conditions prevail, then to pass a mass flux that is nearly the same for all of the above cone angles, the area must remain the same. It appears, therefore, that the shock-body relationship for convex bodies with detached shocks is affected more strongly by the location and geometry of the corner than by the surface con-figuration near the axis of symmetry.

The time history of a representative pressure profile along, a spherical surface of radius $36 \Delta \mathrm{r}$ is shown in $\mathrm{Fig}$. 17 for $\mathrm{M}_{0}=4.63$. It appears that the profile becomes steady after

about 600 iterations; however, as Fig.. 18, a plot of normalized pressure and density vs. time at $r=\Delta r$, shows, about 1,000 iterations are necessary to reach steady-state conditions to within about .5\%. The time history of density shown on this figure is qualitatively similar to that obtained by Burstein [25] for the case of flow over a flat-faced cylinder. Burstein continued the computation for 2,500 iterations, but found no significant change after 1,200 steps. The approach to steady-state will be discussed in more detail when the results for all cone angles are compared.

A plot' of pressure along the axis-surface streamline for the $90^{\circ}$ cone was presented in Fig. 6 together with a similar result for the $50^{\circ}$ cone. These curves graphically illustrate how 
the numerical scheme is able to handle the large jump in pressure from axis to boundary which occurs for an attached shock, and yet results in only a small discontinuity in pressure (which should be absent) in the detached shock case. On the boundary near the vertex, the pressure is within one percent of the value calculated by the normal shock theory.

Plots of pressure vs. angle along spherical surfaces behind the cone are presented in Fig. 19. As in the $80^{\circ}$ case, the gradients become much steeper as the cone is approached. The results are not physically valid more than a few mesh points past the corner, for there the flow separates from the surface and the inviscid theory no longer applies.

\section{B. Three-Dimensional Radiation Effects}

Two calculations were performed to study the threedimensional radiative effects in both detached and attached shock wave configurations. In the first case, hypersonic flow over the $80^{\circ}$ half-angle cone was computed with $M_{0}=47.2, \gamma=1.2$, at the free stream static conditions of $\rho_{0}=3.909 \times 10^{-6}$ and $\mathrm{p}_{\mathrm{o}}=2.915 \times 10^{3}$ (corresponding to an altitude of approximately $58 \mathrm{~km}$ ). The cold wall boundary condition was used (obtained in our formulation by setting $\varepsilon_{\mathbf{S}}=0$ ) and absorptivity was taken to be proportional to the third power of temperature, (obtained from curve fitting the data on page 192 in Ref. 26), i.e.,

$$
\alpha=\alpha_{0} \rho T^{3} \text { per } \mathrm{cm}
$$

where $\alpha_{0}=.40 \times 10^{-10}$. Such absorptivity combined with temperature and density behind $a M_{O}=47.2$ shock results in an optical thickness that is approximately .62 per $\mathrm{cm}$, which means that the shock layer is opaque. The above facts will be helpful in the discussion of the computations of radiative
effects.

The attached shock wave configuration was investigated for the $50^{\circ}$ half-angle cone with $M_{0}=15, \gamma=1.4, \cdot \alpha_{0}=10^{-9}$ and free stream conditions of $\rho_{0}=.184 \times 10^{-4}, p_{0}=.121 \times 10^{5}$. 
The distributions of the square of the speed of sound a quantity proportional to the temperature - and, corresponding to it, energy gain due to radiative transport in front of the $80^{\circ}$ half-angle cone at $r=40 \Delta r$ and $t=400 \Delta t$ are shown in Fig. 20 . These plots demonstrate that the energy loss at the boundaries, adjacent to the cold wall and to the cool gas in front on the shock, greatly exceeds that from the interior of the layer, as it should for an optically thick medium. This is a well known result and we interpret it as an indication that our computations exhibit a qualitatively correct behavior. Fig. 20 also shows that most of the energy radiated out is absorbed immediately in the shock transition layer; note, however, the change in scale from positive to negative directions.

The time history of the temperature profile is shown in Fig. 21, while the steady-state condition is shown in Fig. 22. We see that the radiative energy loss not only reduces the temperature by a factor of approximately four, but also depresses the temperature profile near the wall in accordance with the cold wall boundary condition. The corresponding equilibrium distribution of radiative energy transfer rate has the same general shape as in Fig. 20 but no longer shows such large variations across the shock layer. Also, the values of $Q$ in Figs. 20 and 22 differ by a factor of nearly $10^{7}$, which illustrates the extreme sensitivity of radiative effects to temperature and therefore also to. $\lambda$.

Computations of radiative effects indicate that one calculation of the radiative field, $i . e . ;$ evaluation of $Q_{i, j}$ at all points of the mesh (>5000 values) requires approximately one hour on the Univac I108. However, in the calculation of a steadystate solution significant economies in the required computer time may be effected because almost everywhere the radiative field (obtained from integration) changes slowly in comparison to the flowfield (obtained from differential equations) and therefore $Q^{\prime} s$ need be recomputed only every few hundred fluid dynamical time steps. The radiative transfer rate occasionally changes rapidly near.the vertex, but, as is evident from Fig. 3, there are very few points in this region and consequently, frequent computation of $Q^{\prime}$ s near $r=0$ is not time consuming. The above two properties are exploited in our computer program.

The distribution of radiative energy loss across the shock layer at the position of the corner and of the back face for the $50^{\circ}$ half-angle cone is shown in Fig. 23. We note the nonsymmetric nature of the shock layer and the presence of a local hot spot at the corner. 
The results for the $80^{\circ}$ and $50^{\circ}$ half-angle cones are compared in Fig. 24, which is a plot of normalized temperatures and radiative energy transfer rates along the surface of the cone from the vertex past the corner. We observe in this figure the qualitatively different behavior of the detached and attached shock wave configurations. In the case of the detached shock wave ( $80^{\circ}$ half-angle cone) the temperature is highest at the vertex, decreases to a minimum near the corner, and increases again to a local maximum at the corner. The second maximum occurs at the point where the ray $\theta=\theta_{c}$ intersects the shock wave curving behind the corner of a finite length body; it is higher than at the corner because, as Figs. 21 and 22 show, for flows with radiative effects, the temperature is highest immediately behind the shock.

In contrast to the above behavior of temperature in the case of the detached shock configuration, in the attached shock configuration for the $50^{\circ}$ half-angle cone the temperature increases from a minimum near the vertex to a maximum along the surface and then decreases again towards the corner and beyond. Such behavior obtains because near the vertex the thin shock layer radiates away energy more efficiently than the thicker layer further downstream. The monotone behavior of temperature near the vertex is the vestige of conical similarity of the solution; the ray intersects the shock wave beyond the corner outside of the range of Fig. 24 .

The above results are three-dimensional effects that cannot be obtained from a slab approximation. Unfortunately, the coordinate system set up in Sec. IV and Appendix II for the evaluation of radiation integrals is such that it precludes the possibility of a direct comparison with the slab approximation. (Instead, it is convenient for the specification of rays tangent to the cone.) Such comparison would require a special computer routine which we have not developed in the context of the present investigation.

\section{Flows over Convex Bodies}

Past studies [9] have shown that the presence of a cavity facing the flow may cause periodic oscillations in the flowfield. In order to investigate this phenomenon two convex configurations were considered: (1) conical cavity in a sphere and (2) cylindrical cavity in a cylinder. 


\section{Conical Cavities in a Sphere}

A sphere with conical cavity is easily obtainable with our program by letting the cone half-angle be larger than $90^{\circ}$. Because a large number of time steps are required to obtain information about oscillatory flows only two solutions were computed: a) the $120^{\circ}$ and b) the $135^{\circ}$ half-angle cones; both computations were performed for $M_{O}=20$ at an altitude of $35 \mathrm{~km}$. Unlike in Ref. 9, where the concave body required very smooth corners to obtain a stable numerical solution, in the present treatment the corner is sharp, as is the vertex of the conical cavity.

The shock shapes for the $120^{\circ}$ and $135^{\circ}$ half-angle cones presented in Fig. 25 differ somewhat from those obtained in Ref. 9. Our results indicate that the shock is nearly straight in front of the entire cavity region, whereas in Ref. 9, it has discernible curvature. The sonic lines for $120^{\circ}$ and $135^{\circ}$ cones do not intersect the body at the corner, as in the $80^{\circ}$ and $90^{\circ}$ cone cases, but about $6^{\circ}$ behind the corner. For this geometry both the distance from the sonic line-shock intersection to the sonic line-body intersection are larger than the constant value which obtains for the $80^{\circ}$ and $90^{\circ}$ cones.

The approach of the flowfield to steady-state in the case of the $120^{\circ}$ half-angle cone is presented in Fig. 26 and compared to similar results for, the $50^{\circ}, 80^{\circ}$, and $90^{\circ}$ half-angle cones. The plots in Fig. 26 represent the time history normalized pressure on the surface, one mesh point away from the vertex, for each cone angle. The results indicate that the solution corresponding to flow over a spherical body with a conical cavity $\left(120^{\circ}\right.$ half-angle cone) undergoes large regular oscillations unlike solutions for the other cone geometries studied. The oscillations, however, appear to be strongly damped and do not persist as was found in. Ref. 9. This observation should not be viewed as a contradiction, however, because the geometries considered and methods used. in the two analyses are quite different. Rather, it points out a need for further investigations in this direction with a method that has much Iess numerical viscosity.

Because Ref. 9 shows that steady-state oscillations are more likely to occur when the cavity depth is increased, a computation was made for a cone of $135^{\circ}$ half-angle for which the cavity depth is nearly $40^{\circ}$ larger than for the $120^{\circ}$ cone. The shock shape and the sonic line are shown in Fig. 25 and the time 
history of pressure is compared in Fig. 27 with the corresponding plot for the $120^{\circ}$ cone. Again, large oscillations are evident, but they are eventually damped. Results show that the ratio of the oscillation periods for $135^{\circ}$ and $120^{\circ}$ cones is equal to the ratio of the vertex to shock distances for these cones. Thus, the oscillation may be viewed as a disturbance travelling back and forth from the vertex to the shock and continuously dissipating energy.

To further verify some of the observations and conclusions about flows over convex bodies, a different shape was considered. It consisted of the $50^{\circ}$ half-angle cone to which a protruding spherical flange was added as shown in Fig. 28. This particular shape was dictated by the coordinate system and the structure of the basic cone program. It was motivated by the desire to examine a concavebody with the minimum possible 'artificial viscosity' in the. flowfield, so as to reduce the damping of any oscillations which occur. The 'artificial viscosity' which is related to the mesh dimension in $\theta$ and $r$ directions, is here less by a factor of three from that for the $135^{\circ}$ cone. The resulting shock and sonic line are shown in Fig. 28. Along the axis, the standoff distance is about $65 \%$ of the body radius. Also, as in the case of previously considered concave bodies, the shock curvature is zero near the axis and for some distance away from the axis. Fig. 29 is a plot of pressure vs. time for a point located on the axis two radial mesh units from the vertex. Evidently, oscillatory behavior does occur, but is damped as for other concave bodies. To significantly reduce the 'artificial viscosity', and further investigate this interesting phenomenon, a higher-order accurate scheme would be necessary.

\section{Cylindrical Cavities}

To study flows over convex shapes with different artificial viscosity and without spurious effects of a nonuniform mesh, two computations were made of flow over a hollow cylinder. As Fig. 30 shows, the configuration has thin walls, and is open at one end and closed at the other. The free stream conditions for these computations are:

$$
\begin{aligned}
M_{0}=10 ; p_{0} & =.1 \cdot 10^{5} \frac{\text { dynes }}{\mathrm{cm}^{2}} ; \rho_{0}=.1 \cdot 10^{-4} \frac{\text { grams }}{\mathrm{cm}^{3}} \\
\Delta t & =.16 \cdot 10^{-5} \mathrm{sec} . ;
\end{aligned}
$$


the cylinder radius is $37 \mathrm{~cm}$ and wall thickness is zero. The two computations correspond to cavity depths of $40 \mathrm{~cm}$. and $68 \mathrm{~cm}$.

Shock and sonic line shapes for both cases are shown in Fig. 30; after about 5000 iterations, they become essentially independent of cavity depth. The shock is nearly straight for a distance of almost half-radius from the axis, then bends to match the rapid changes which occur near the corner and finally straightens out again as it weakens into a Mach wave. The sonic line intersects the cylinder outer surface four centimeters beyond the corner. This places the cylinder edge within the subsonic (elliptic) region and signifies that the edge geometry has an effect on the flow upstream of the cylinder. In the previous case of flow over concave cones, a similar effect was noted. However, there still exists the possibility that the location of the edge within the subsonic region is a purely numerical effect and an experimental check should be made to resolve this matter.

The time history of the shock position on the axis is shown in Fig. 31. For the initial value problem presented here the shock exhibits damped oscillations before settling down to its steady-state position. Results show that the ratio of the distances from the base of the cavity to the shock equilibrium position is equal to the ratio of the oscillation periods. Thus, for the present geometry, a characteristic length for oscillation is not the tube depth (a strictly geometric property), but rather the tube base-shock distance (a geometric-thermodynamic property). From simple acoustic theory it is known that a wave moving back and forth in a tube open at one end and closed at the other has a wavelength equal to four times the tube depth; present results yield a measured wavelength equal to about 4.5 times the tube base-shock distance. Thus, in this problem the tube base-shock distance may be considered to be the 'effective' length of an open-end tube.

Plots of pressure along the stagriation (axis) streamline for both cylinders at $t=5200 \Delta t$ are shown in Fig. 32 . For the $40 \mathrm{~cm}$. deep cylinder the pressure increases monotonically towards the base, whereas for the $68 \mathrm{~cm}$. deep cylinder, the pressure reaches a maximum inside the cavity. In both cases, the pressure at the wall is less than the one-dimensional normal shock stagnation value of $1.29 \cdot 10^{6} \frac{\text { dynes }}{\mathrm{cm}^{2}}$. A drop in stagnation pressure denotes the presence of an entropy production process. As will be seen on the next figure, this process is the formation of vortices within the cylinder. 
The streamline pattern inside the $40 \mathrm{~cm}$. deep cavity after 5200 time steps is shown in Fig. 33. The flow in the region near the cavity mouth appears to drive the flow near the base. The driven flow contains a vortex, which changes its position and intensity with time. We conjecture. that after a sufficiently long time, the driven flow will form a closed cell and continuously circulate in a clockwise direction. At the time shown, mass from the driven region exits the cylinder, which correlates well with a pressure decrease in that region.

Fig. 34 illustrates how well the scheme handles the large gradients near the cylinder edge. It contains plots of pressure vs. distance in the axial direction along surfaces in the vicinity of the cylinder wall. The solid curves correspond to surfaces inside the cylinder, including the cylinder inner wall, and the dotted curves correspond to surfaces outside the cylinder, including the cylinder outer wall. On $y=37 \Delta y$, the cylinder surface, the curve which represents the outer surface shows a continuous and smooth expansion; the curve which represents the inner surface shows a clear distinction between the relatively high velocity, low pressure fluid outside of the cavity and nearly stagnant fluid inside the cavity.

VI. SUMMARY AND CONCLUSIONS

This memorandum describes a numerical approach useful in the computation of hypersonic flowfields over conical shells with spherical afterbodies and thin-walled open-ended cylinders. It discusses the theoretical background of the method, the numerical scheme used in its execution, and presents numerical results. In particular, results for several values of freestream Mach number, $\mathrm{M}_{\mathrm{O}^{\prime}}$ for cone half-angles of $50^{\circ}, 80^{\circ}, 90^{\circ}$ (plane face), $120^{\circ}$ (conical cavity), $135^{\circ}$, and for thin-walled cylinders of different depths are described.

Four solutions were calculated for the flow past the $50^{\circ}$ cone. One was obtained with the solid surface boundary condition; it demonstrated that the method is capable of handling the large jump across the shock attached at the vertex of the cone and of producing results acceptable for many purposes. The second one was obtained with large mass injection at the surface (injection momentum $=40 \%$ of the free stream momentum); it demonstrated that the method is capable of following the transition from attached to detached shock configurations. The third solution included the three-dimensional radiative transfer effects for $M_{0}=15$ and showed that the slab approximation is subject to 
large errors near the cone vertex and near the rear corner. In the fourth computation a spherical flange was added to the cone to simulate a concave forebody and the results indicated that the calculation process for this shape converges in an oscillatory manner in contrast to the monotone convergence for a convex forebody.

For the $80^{\circ}$ and $90^{\circ}$ cones solutions were obtained at $\mathrm{M}_{\mathrm{O}}=4.63,20$, and 47.2 . The $\mathrm{M}_{\mathrm{O}}=4.63$ results show

excellent agreement with the experimental observations while the $\mathrm{M}_{\mathrm{O}}=20$ results agree well with the calculations by the method of Integral Relations. The $80^{\circ}$ cone was also used to compute the effects of changing $\gamma$ and of the three-dimensional radiative energy transfer for the $\mathrm{M}_{\mathrm{O}}=47.2$ flow. The results

demonstrate the correct compression of the shock layer at constant pressure for decreasing $\gamma$, large radiative energy loss near cold surfaces, and significant departures from slab approximation near surface discontinuities.

Solutions describing flow over conical cavities, i.e., for cone half-angles of $120^{\circ}$ and $135^{\circ}$ were obtained at $\mathrm{M}_{\mathrm{O}}=20$. They display strongly damped oscillatory behavior with the ratio of periods for the $120^{\circ}$ and $135^{\circ}$ cases equal to the ratio of the corresponding shock stand-off distances measured from the cone vertex.

Two computations were made for a thin-walled cylinder at $M_{0}=10$, one for a hole depth of $40 \mathrm{~cm}$. (hole radius $=37 \mathrm{~cm}$ ) and the other for a hole depth of $68 \mathrm{~cm}$. As in the case of the concave conical bodies, the numerical scheme converges in a damped oscillatory manner with the ratio of the periods of oscillation for the two cases being identical with the ratio of shock-base of hole distances measured along the cylinder axis.

In closing, it should be emphasized that the object of the calculations was to obtain steady-state features. Use - of the unsteady technique was only a means to this end. While the approach to steady-state from an arbitrary initial condition may exhibit interesting facets, the intermediate results do not necessarily have physical significance. Thus the vortex formation and accompanying damped oscillations, dependent on the large 'artificial viscosity', should be considered as numerical phenomena. Higher order accurate schemes with much lower dissipation are necessary to differentiate between numerical and 
physical effects. The reliable physical results obtained with the first-order accurate technique are the steady-state shock shapes and pressure plots.

directions Future work in this area may proceed in several of the of the results by employing more accurate differencing schemes and/or by devising better numerical procedures for the treatment of surface and internal boundaries, as required for injection effects. It should be noted that the correct specification of boundary conditions for the finite-difference scheme used in the present work is still an open question in numerical analysis. Another direction for future investigations is to perform parametric studies in regions of particular interest, e.g., near the critical cone angle or around the transition from convex to concave body (near $\theta_{C}=90^{\circ}$ ). Also, when atmospheric entry conditions of particular interest are identified, computations may be performed for the purpose of determining the aerothermodynamic characteristics of entry vehicle shapes described by different cone half-angles. These properties are useful for the study of

1013-RNK-ajj

I031-IOB-sje

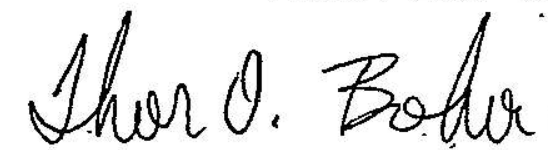

I. O. Bohachevsky

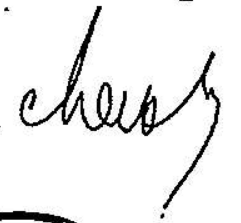

Attachments

References

Appendices I \& II

Figures I-34

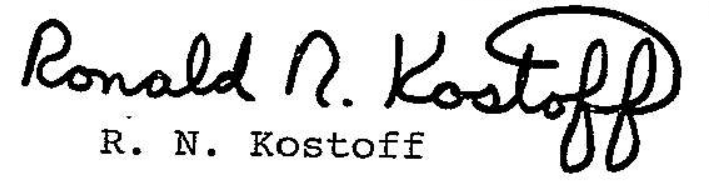


REFERENCES

1. Kostoff, et al., "Experiment Payloads for a Manned Mars Flyby Mission", Bellcomm TR-67-233-1, May 15, 1967; or Kostoff, et al., "Experiment Payloads for Manned Encounter Missions to Mars and Venus", Bellcomm TR-68-710-2, February 21,1968 .

2. Bohachevsky, I. O. and Kostoff, R. N., "Numerical Study of Reentry Phenomena", Bellcomm Memo for File, October 11, 1968 .

3. Schneider, P. J., Mauer, R. E. and Strapp, M. G., "TwoDimensional Transpiration Cooling", AIAA Paper 69-96, January, 1969.

4. Jarvinen, P. O., Adams, R. H., "The Effects of RetroRockets on the Aerodynamic Characteristics of Conical Aeroshell Planetary Entry Vehicles", AIAA Paper 70-219, January, 1970 .

5. Bohachevsky, I. O. and Rubin, E. I., "A Direct Method for Computation of Nonequilibrium Flows with Detached Shocks", AIAA Journal, 4 , pp. 600-607 (1966).

6. Bohachevsky, I. O. and Mates, R. E., "A Direct Method for Calculation of the Flow about an Axisymmetric Blunt Body at Angle of Attack", AIAA Journal, $\underline{4}$, pp. 775-782 (1966).

7. Crocco, I., "A Suggestion for the Numerical Solution of the Steady Navier-Stokes Equations", AIAA Journal; $\underline{3}$, pp. 1824-1832 (1965).

8. Godunov, S. K., Zabordin, A. O. and Prokopov, G. P., "A Finite Difference Scheme for Two-Dimensional Unsteady Flow with a Detached Shock Wave", Z. Vychyslitelnoi Mat. i. Mat. Fiziki I, pp. 1020-1050 (1961); also Cornell Aeronautical Lab. transl.

9. Bastianon, R., "Steady and Unsteady Solution of the Flow Field Over Concave Bodies in Supersonic Free Stream", AIAA. Paper 68-946, June, 1968.

10. Bohachevsky, I. O., "Computation of Three-DimensionaI Supersonic Flows with shock Waves", Bellcomm Technical Memorandum, TM70-1011-7, August 6, 1970. 
11. Jackomis, W. N. and Zumwalt, G. W., "Transient Flow Field Analysis of a Plane Blast Wave Intercepting a stationary Cone at Zero Angle of Attack", Sandia Corporation Report, SC-DC-66-1329, August, 1965.

12. Lax, P. D., "Weak Solutions of Nonlinear Hyperbolic Equations and their Numerical Computation", Comm. Pure Appl. Math., 7, pp. 159-193 (1954).

13. Zeldovich, U. B., and Raizer, Y. P., "Physics of Shock Waves and High-Temperature Hydrodynamic Phenomena", Chap. 3, Vol. 1, Academic Press, New York, 1966.

14. Richtmyer, R. D. and Morton, K. W., "Difference Methods for Initial Value Problems", 2nd Ed., p. 300, Interscience Publishers, New York, 1967.

15. Iax, P. and Wendroff, B., "Systems of Conservation Laws", Comm. Pure Appl. Math., XIII, pp. 217-237 (1960).

16. Rusanov, V. V., "Finite Difference Schemes of Third Order Accuracy. for Continuous Computation of Discontinuous Solutions", Inst. App. Math., AN USSR Preprint, 1967. Also: Soviet Math. DokI. 9 No. 3 (1968).

17. Courant, R., Friedrichs, K.'O. and Lewy, H., "Uber die Partiellen Differenzengleichungen der Mathematischen Physik", Math. Ann., 100, p. 32, 1928.

18. Anderson, J. D., Jr., "An Engineering Survey of Radiating Shock Layers", AIAA Journal, 7, pp. 1665-1675 (1969).

19. Lighthi11, M. J., "Dynamics of a Dissociating Gas, Part 2, Quasi-Equilibrium Transfer Theory", J. of Fluid Mech., $\underline{8}$, pp. 16I-182 (1960).

20. Sims, J. I., "Tables for Supersonic Flow Around Right Circular Cones at Zero Angle of Attack", NASA SP-3004,.1964.

21. Wallace, J. and-Kemp, N. H., "Similarity Solutions to the Massive Blowing Problem", AIAA Journal, 7, pp. 151.7-1523 (1969) .

22. Campbel1, J. F. and Howel1, D. F., "Supersonic Aerodynamics of Large-Angle Cones", NASA TN-D-4719, August, 1968. 
23. Hayes, W. and Probstein, R., "Hypersonic Flow Theory", I, Academic Press, New York, 1966.

24. Ames Research Staff, "Equations, Tables, and Charts for Compressible Flow", NACA. Report 1135, 1953.

25. Burstein, S. Z., "Finite Difference Calculations for Hydrodynamic Flows Containing Discontinuities", Journal of Computational Physics, 1 , No. 2, November, 1966.

26. Penner, S. S. and Olfe, D. B., "Radiation and Reentry", Academic Press, New York, 1968. 


\section{APPENDIX I}

\section{DIFFERENCE EQUATIONS IN SPHERICAL COORDINATES}

A. Coordinates of the grid point $(i, j)$ are:

$$
\begin{gathered}
\left(\theta_{i}, r_{j}\right) \text { with } \theta_{i}=(i-3) \Delta \theta+\theta_{C} \\
r_{j}=(j-1) \Delta r
\end{gathered}
$$

where $\theta_{C}$ is the cone half-angle and the angular increment $\Delta \theta$ appropriate to each region is used (see Fig. 3),

B. Superscripts denote quantities evaluated at time $t+\Delta t$ and subscripts those evaluated at time $t$.

Equations (1) - (4) and (5) - (8) are replaced by the following finite difference analogues.

Eq. (1), Conservation of Mass:

$$
\begin{aligned}
& \left(\rho r^{2} \sin \theta\right)^{i, j}=r_{j}^{2} \sin \theta\left[c_{i} \rho_{\cdot i, j}+c_{2} \rho_{i, j+1}+c_{3} \rho_{i, j-1}+\right. \\
& \left.+C_{4} \rho_{i+1, j}+C_{5} \rho_{i-1, j}\right]- \\
& -\frac{\Delta t}{2 \Delta r} \frac{\sin \Delta \theta \cos \Delta \theta}{\Delta \theta}\left[\left(\rho \operatorname{ur}^{2} \sin \theta\right)_{i, j+1}-\left(\rho u r^{2} \sin \theta\right)_{i, j-1}\right]- \\
& -\frac{\Delta t}{2 \Delta r}\left[(\rho \operatorname{vr} \sin \theta)_{i+1, j}-(\rho \operatorname{vr} \sin \theta)_{i-1, j}\right.
\end{aligned}
$$


Eq. (2), Conservation of $r$-momentum:

$$
\begin{aligned}
& \left(\rho \operatorname{ur}^{2} \sin \theta\right)^{i, j}=r_{j}^{2} \sin \theta_{i}\left[c_{i}(\rho u)_{i, j}+c_{2}(\rho u)_{i, j+1}+\right. \\
& +c_{3}(\rho u)_{i, j-1}+c_{4}\left[\rho u_{i+1, j} \cos \Delta \theta-(\rho v)_{i+1, j} \sin \Delta \theta\right]+ \\
& +C_{5}\left[(\rho u)_{i-1, j} \cos \Delta \theta+(\rho v)_{i-1, j} \sin \Delta \theta\right]- \\
& -\frac{\Delta t}{2 \Delta r} \frac{\sin \Delta \theta \cos \Delta \theta}{\Delta \theta}\left[\left(\rho u^{2}+p\right)\left(r^{2} \sin \theta\right)_{i, j+1}-\left(\rho u^{2}+p\right)\left(r^{2} \sin \theta\right)_{i, j-1}\right]- \\
& -\frac{\Delta t}{2 \Delta \theta}\left\{(r \sin \theta)_{i+1, j}[(\rho v)(u \cos \Delta \theta-v \sin \Delta \theta)]_{i+1, j}-\right. \\
& \left.-(r \sin \theta)_{i-1, j}[(\rho v)(\dot{u} \cos \Delta \theta+v \sin \Delta \theta)]_{i-1, j}\right\}+ \\
& +\Delta t \frac{\sin \Delta \theta}{\Delta \theta}\left[(\operatorname{pr} \sin \theta)_{i+1, j}+(\operatorname{pr} \sin \theta)_{i-1, j}\right]
\end{aligned}
$$

Equation (3), Conservation of $\theta$-momentum:

$$
\begin{aligned}
& \left(\rho v r^{2} \sin \theta\right)^{i, j}=r_{j}^{2} \sin \theta_{i}\left\{C_{i}(\rho v)_{i, j}+C_{2}(\rho v)_{i, j+1}+C_{3}(\rho v)_{i, j-I}+\right. \\
& +c_{4}\left[(\rho v)_{i+1, j} \cos \Delta \theta+(o u)_{i+1, j} \sin \Delta \theta\right]+ \\
& \left.+C_{5}\left[(\rho v)_{i-1, j} \cos \Delta \theta-(\rho u)_{i-1, j} \sin \Delta \theta\right]\right\}- \\
& -\frac{\Delta t}{2 \Delta r} \frac{\sin \Delta \theta \cos \Delta \theta}{\Delta \theta}\left[\left(\rho u v r^{2} \sin \theta\right)_{i, j+1}-\left(\rho u v r^{2} \sin \theta\right)_{i, j-1}\right]- \\
& -\frac{\Delta t}{2 \Delta \theta}\left\{( r \operatorname { s i n } \theta ) _ { i + 1 , j } \left[(\rho v)(u \sin \Delta \theta+v \cos \Delta \theta)_{i+1, j}-\right.\right. \\
& \left.-(r \sin \theta)_{i-1, j}[(\rho v)(-u \sin \Delta \theta+v \cos \Delta \theta)]_{i-1, j}\right\}- \\
& -\frac{\Delta t}{2 \Delta \theta} r_{j} \sin \theta_{i} \cos \Delta \theta\left(p_{i+1, j}-p_{i-1, j}\right)
\end{aligned}
$$


AI -3

Eq. (4), Conservation of Energy:

$$
\begin{aligned}
& {\left[\left(\frac{1}{2} q^{2}+\frac{1}{\gamma-I} p\right) r^{2} \sin \theta\right]^{i, j}=r_{j}^{2} \sin \theta_{i}\left[c_{I}\left(\frac{1}{2} p q^{2}+\frac{1}{\gamma-1} p\right)_{i, j}+\right.} \\
& +c_{2}\left(\frac{1}{2} \rho q^{2}+\frac{1}{\gamma-1} p\right)_{i, j+1}+c_{3}\left(\frac{1}{2} \rho q^{2}+\frac{1}{\gamma-1} p\right)_{i, j-1}+ \\
& \left.+c_{4}\left(\frac{1}{2} \rho q^{2}+\frac{1}{\gamma-1} p\right)_{i+1, j}+c_{5}\left(\frac{1}{2} \rho q^{2}+\frac{1}{\gamma-1} p\right)_{i-1, j}\right]- \\
& -\frac{\Delta t}{2 \Delta r} \frac{\sin \Delta \theta \cos \Delta \theta}{\Delta \theta}\left\{\left[\left(\frac{1}{2} q^{2}+\frac{\gamma}{\gamma-1} \frac{p}{\rho}\right) \rho u r^{2} \sin \theta\right]_{i, j+1}-\right. \\
& \left.-\left[\left(\frac{1}{2} q^{2}+\frac{\gamma}{\gamma-1} \frac{p}{\rho}\right) \rho u r^{2} \sin \theta\right]_{i, j-1}\right\}- \\
& -\frac{\Delta t}{2 \Delta \theta}\left\{\left[\left(\frac{1}{2} q^{2}+\frac{\gamma}{\gamma-1} \frac{p}{\rho}\right) \rho v r \frac{\sin \theta]}{i+1, j}\right.\right. \\
& \left.-\left[\left(\frac{1}{2} q^{2}+\frac{\gamma}{\gamma-1} \frac{p}{\rho}\right) \rho v r \sin \theta\right]_{i-1, j}\right\}
\end{aligned}
$$

On the axis, $\theta=\pi$, Eqs. (5) - (8) are replaced in a similar fashion.

Eq. (5), Conservation of Mass:

$$
\begin{aligned}
& \left(\rho r^{2}\right)^{i, j}=r_{j}^{2}\left[C_{1}{ }_{i, j}+C_{2}{ }_{i, j+1}+C_{3} \rho_{i, j-1}+C_{4}{ }_{i+1, j}+C_{5} \rho_{i-1, j}\right] \\
& -\frac{\Delta t}{2 \Delta r} \frac{\sin \Delta \theta}{\Delta \theta}\left[\left(\rho u r^{2}\right)_{i, j+1}-\left(\rho u r^{2}\right)_{i, j-1}\right]_{-} \\
& -\frac{\Delta t}{2 \Delta \theta}\left[(2 \rho v r)_{i+1, j}-(2 \rho v r)_{i-1, j]}\right.
\end{aligned}
$$




$$
\mathrm{AI}-4
$$

Eq. (6), Conservation of r-momentum:

$$
\begin{aligned}
& \left(\rho u r^{2}\right)^{i, j}=r_{j}^{2}\left[c_{1}(\rho u)_{i, j}+c_{2}(\rho u)_{i, j+I}+c_{3}(\rho u)_{i, j-1}+\right. \\
& +c_{4}\left[(\rho u)_{i+1, j} \cos \Delta \theta-(\rho v)_{i+1, j} \sin \Delta \theta\right]+ \\
& +c_{5}\left[(\rho u)_{i-1, j} \cos \Delta \theta+(\rho v)_{i-1, j} \sin \Delta \theta\right]- \\
& -\frac{\Delta t}{2 \Delta r} \frac{\sin \Delta \theta}{\Delta \theta}\left\{\left[\left(\rho u^{2}+p\right) r^{2}\right]_{i, j+1}-\left[\left(\rho u^{2}+p\right)^{2}\right]_{i, j-1}\right\}- \\
& -\frac{\Delta t}{\Delta \theta}\left[[(r \rho v)(u \cos \Delta \theta-v \sin \Delta \theta)]_{i+1, j}-\right. \\
& -\left[(x \rho v)(u \cos \Delta \theta+v \sin \Delta \theta)_{i-I, j}+\Delta t 2(p r)_{i, j}\right.
\end{aligned}
$$

Eq. (7), Conservation of $\theta$-momentum:

$$
v^{i, j}=0
$$

Eq. (8), Conservation of Energy :

$$
\begin{aligned}
& {\left[\left(\frac{1}{2} p q^{2}+\frac{1}{\gamma-1} p\right) r^{2}\right]^{i, j}=r_{j}^{2}\left[c_{I}\left(\frac{1}{2} p q^{2}+\frac{1}{\gamma-I} p\right)_{i, j}+\right.} \\
& +c_{2}\left(\frac{1}{2} p q^{2}+\frac{1}{\gamma-1} p\right)_{i, j+1}+c_{3}\left(\frac{1}{2} p q^{2}+\frac{1}{\gamma-1} p\right)_{i, j-1}+ \\
& \left.+c_{4}\left(\frac{1}{2} \rho q^{2}+\frac{1}{\gamma-I} p\right)_{i+1, j}+c_{5}\left(\frac{1}{2} \rho q^{2}+\frac{1}{\gamma-1} p\right)_{i-1, j}\right]- \\
& -\frac{\Delta t}{2 \Delta r} \frac{\sin \Delta \theta}{\Delta \theta}\left\{\left[\left(\frac{1}{2} q^{2}+\frac{\gamma}{\gamma-1} \frac{p}{\rho}\right) \rho u r^{2}\right]_{i, j+1}-\left[\left(\frac{1}{2} q^{2}+\frac{\gamma}{\gamma-I} \frac{p}{\rho}\right) \rho u r^{2}\right]_{i, j-1}\right\}- \\
& -\frac{\Delta t}{\Delta \theta}\left\{\left[\left(\frac{1}{2} q^{2}+\frac{\gamma}{\gamma-1} \frac{p}{\rho}\right) \rho v r\right]_{i+1, j}-\left[\left(\frac{I}{2} q^{2}+\frac{\gamma}{\gamma-I} \frac{p}{\rho}\right) \rho v r\right]_{i-1, j}\right\}
\end{aligned}
$$

In the above equations, $c_{1}, c_{2}, C_{3}, c_{4}$, and $c_{5}$ are given by:

$$
\begin{aligned}
& c_{1}=1-\frac{r_{j}^{2}+\Delta r^{2}}{2 r_{j}^{2}}-\frac{\cos \Delta \theta}{2} \\
& c_{2}=\frac{\left(r_{j}+\Delta r\right)^{2}}{4 r_{j}^{2}}
\end{aligned}
$$




$$
\begin{aligned}
& c_{3}=\frac{\left(r_{j}-\Delta r\right)^{2}}{4 r_{j}^{2}} \\
& c_{4}=\frac{\sin \left(\theta_{i}+\Delta \theta\right)}{4 \sin \theta_{i}} \\
& c_{5}=\frac{\sin \left(\theta_{i}-\Delta \theta\right)}{4 \sin \theta_{i}}
\end{aligned}
$$

It is easily seen that $C_{1}$ and the differences $c_{k}-\frac{1}{4}$, $k>1$, are of the second order in mesh size and therefore do not affect the first order accuracy of the scheme except very near the vertex. The purpose of the $C_{k}{ }^{\prime} s$ is to introduce into the computations numerical damping in the form of the Iaplacean. 


\section{APPENDIX II}

\section{NUMERICAI ROUTINE FOR CAI,CULATING RADIATION INTEGRALS}

In this Appendix we elucidate the computation of radiative effects in more detail by listing the instructions for the preparation of a computer program routine which calculates the radiation integrals. Because it depends essentially only on the fact that the coordinate system is spherical, this routine is also applicable to the evaluation of the radiative contribution in the determination of global temperature distribution in planetary atmospheres and is presently being used for that purpose.

The computational steps are as follows (see Fig. 2).

I. At each point $\left(\theta_{i}, r_{j}\right)$ select the values of $d \psi_{n}$ and $d \lambda_{m}$ so that $\psi_{\mathrm{n}}=(\mathrm{n}-1) \mathrm{d} \psi_{\mathrm{n}}$ and $\lambda_{\mathrm{m}}=(\mathrm{m}-1) \mathrm{d} \lambda_{\mathrm{m}}$.

II. Check the value of $r_{j}$

A. When $r_{j} \underline{s}_{C}$ go to step III.

B. When $r_{j}>r_{C}$ check the value of $\psi$.

1. If $\psi \leq 1 / 2 \pi$ select $d R$ and proceed to calculate radiative contribution as outlined in step IV.

2. If $\psi>1 / 2 \pi$ the ray may hit the spherical back surface of the cone at the distance given by:

$$
R_{b s}=-r_{j}\left(\cos \psi+\sqrt{\left(\frac{r_{c}}{r_{j}}\right)^{2}-\sin ^{2} \psi}\right) \cdot
$$

To decide that, check the value of ${ }^{\theta}$ bs calculated

as in IV-A at the distance $R_{b s}$ from $r_{j}$ :

a. If $\theta_{b s} \geq \theta_{c}$ the ray misses the surface and the program proceeds to step IV.

b. If $\underline{\theta}_{\mathrm{bc}}<\underline{\theta}_{\mathrm{c}}$ the ray hits the back surface; divide $R_{b s}$ into an integer number of steps $\mathrm{dR}$ and calculate radiative contribution as in step IV. 
III. When $r_{j} \leq r_{c}$ check the value of $\theta_{i}$.

A. When $\underline{\theta}_{C}<\theta i \leq \pi-\theta$ compute the value of the angle $\lambda_{s}$, in the range $\pi<\lambda_{s} \leq \frac{1}{2} \pi$, at which the rays become tangent to the cone surface from:

$$
\sin \lambda_{s}=\frac{\sin \theta_{C}}{\sin \theta_{i}}
$$

B. Check the value of $\lambda_{m}$

I. If $\lambda \underbrace{}_{-\lambda}$ select $d R$ and proceed to calculate radiative contribution as outlined below in step IV.

2. If $\lambda_{m}>\lambda$ compute:

$$
\begin{aligned}
& c_{1}=\left(\cos \psi_{n} \cos \theta_{i}-\sin \psi_{n} \sin \theta_{i} \cos \lambda_{m}\right)^{2}-\cos ^{2} \theta_{c} \\
& c_{2}=\cos ^{2} \theta_{i}-\cos ^{2} \theta_{c} \\
& c_{3}=\cos \theta_{i}{ }^{\sin \theta_{i}} \sin _{n}{ }^{\cos \lambda_{m}} \\
& R_{S}^{ \pm}=\frac{r_{j}}{c_{1}}\left[c_{3}-c_{2} \cos _{n} \pm \sin _{n} \cos \theta_{C}\left(c_{2}+\sin ^{2} \theta_{i} \cos ^{2} \lambda_{m}\right)^{1 / 2}\right]
\end{aligned}
$$

and then

a. if both roots are negative disregard them and compute radiative contribution as in step IV below.

b. if the two roots have different signs, i.e., $\mathrm{R}_{\mathrm{S}}^{+}>0$ and $\mathrm{R}_{\mathrm{S}}^{-}<0$; choose the positive value for $R_{s}$,

c. if both roots are positive, i.e., $\mathrm{R}_{\mathrm{S}}^{+}>0$ and $-R_{S}^{-}>0$, choose the smaller value for $R_{s}$; in cases $b$ and $c$ divide $R_{S}$ into $d R$ intervals and proceed to calculate the radiation contribution as outlined below in step IV. 


\section{AII-3}

C. When $\pi-\theta \quad c<\theta \leq \pi$ then check the value of $\psi_{n}$.

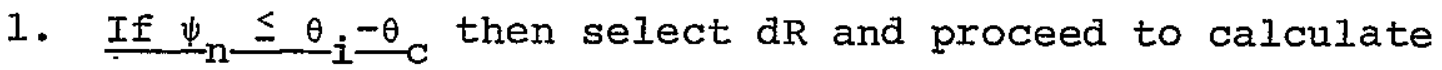
the radiation contribution as outiined in step IV.

2. If $\psi_{n}>\theta+\frac{-\theta}{C}$ then compute $C_{1}, C_{2}, C_{3}$, and $R_{S}^{ \pm \cdot}$ as in III-B2 above.

a. If the two roots have different signs, i.e., $\mathrm{R}_{\mathrm{S}}^{+}>0$ and $\mathrm{R}_{\mathrm{S}}^{-}<0$, or if both roots are negative, then disregard them, select $d R$ and proceed to calculate the radiation contribution as outlined in step IV.

b. If both roots are positive, i.e., $\mathrm{R}_{\mathrm{S}}^{+}>0$ and $R_{S}^{-}>0$, then choose for $R_{S}$ the larger value, divide $R_{S}$ into $d R$ intervals and proceed to calculate the radiation contribution as outlined in step IV.

IV. To evaluate radiative contribution along each ray $\left(\psi_{n}, \lambda_{m}\right)$
at the point $\left(\theta_{i}, r_{j}\right), i, e, k=0$

A. Compute:

$$
\begin{aligned}
& R_{\ell}=R_{\ell-1}+d R \\
& r=\left[R_{\ell}^{2}+r_{j}^{2}+2 R_{\ell} r_{j} \cos \psi_{n}\right]^{1 / 2} \\
& \cos \theta=\frac{r_{j}+R_{\ell} \cos \psi_{n}}{r} \cos \theta_{i}-\frac{R_{\ell}}{r} \sin \psi_{n} \cos \lambda_{m} \sin \theta_{i}
\end{aligned}
$$

B. At each $R_{\ell}$, i.e., at each point $(\theta, r)$ determine by interpolation the values of $p, \rho$, and $T$; then compute:

$$
\begin{aligned}
& \text { 1. } \alpha_{\ell} \text { and } B_{\ell} \\
& \text { 2. } \tau_{\ell}=\tau_{\ell-1}+\alpha_{\ell} d R \\
& \text { 3. } A_{\ell}=A_{\ell-1}+\alpha_{\ell} B_{\ell} e^{-\tau} \ell d R
\end{aligned}
$$




\section{AII-4}

c. Now check the program branch:

1. If there is no value for $R_{s}$, continue until $r_{m}$.

2. If there is a value for $R_{S}$, terminate at $R=R_{I}=R_{S}$ and compute the surface emission

$$
A_{S}=\varepsilon_{S} B_{S} e^{-\tau} s \cos (R \cdot n)
$$

where

$$
\begin{aligned}
& \text { a. for } \underline{r}_{j}<r_{C} \text { (on } \theta=\theta_{c^{\prime}} r<r_{c} \text { ): } \\
& \cos (R \cdot n)=\left|\cos \left(\frac{I}{2} \pi-\theta_{C}+\theta_{i}-\psi\right) \cos (\lambda+\chi)\right| \\
& \text { with } x=\operatorname{arc} \sin \left|\frac{R \sin \psi \sin \lambda}{r \sin \theta_{C}}\right| \\
& 0 \leq x \leq \frac{1}{2} \pi \\
& \text { b. } \underline{\text { for }} r_{j}>r_{C}\left(\text { on } r=r_{c}, \theta<\theta_{c}\right) \text { : } \\
& \cos (R \cdot n)=\left|\cos \psi \cos \left(\theta-\theta_{i}\right)\right|
\end{aligned}
$$

V. Carry out step IV for every pair $\left(\psi_{n}, \lambda_{m}\right)$, store each result and sum over all directions to obtain:

$$
A_{i, j}=\alpha_{i, j} \sum_{m} \sum_{n}\left(A_{L}^{n, m}+A_{s}^{n, m}\right) \sin \psi_{n} d \psi_{n} d \lambda_{m}
$$




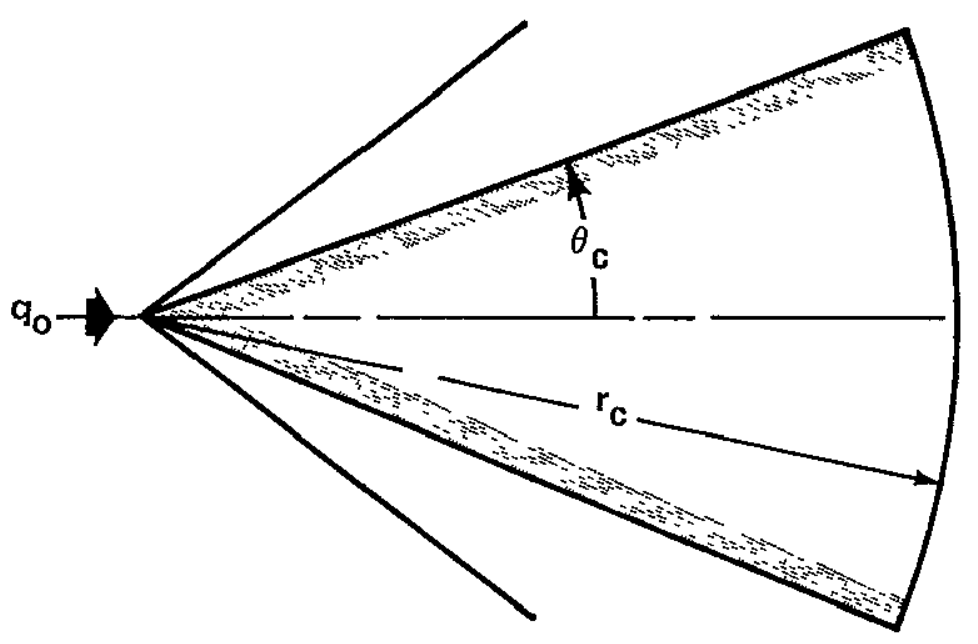

A

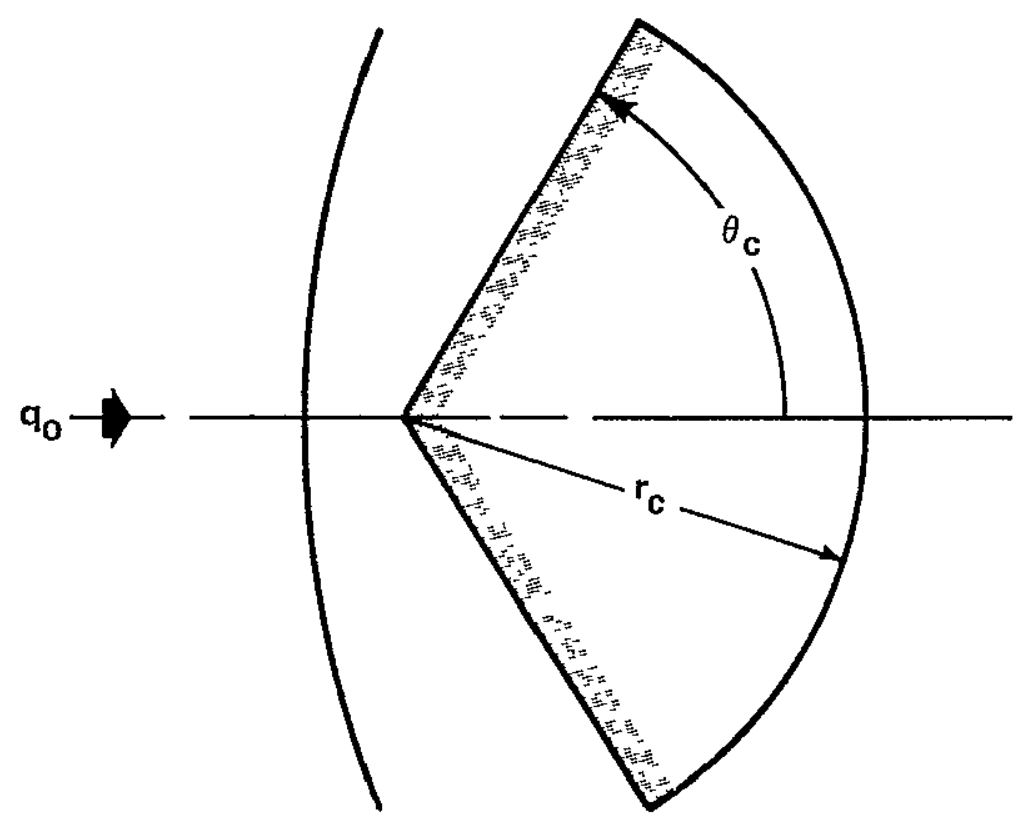

B

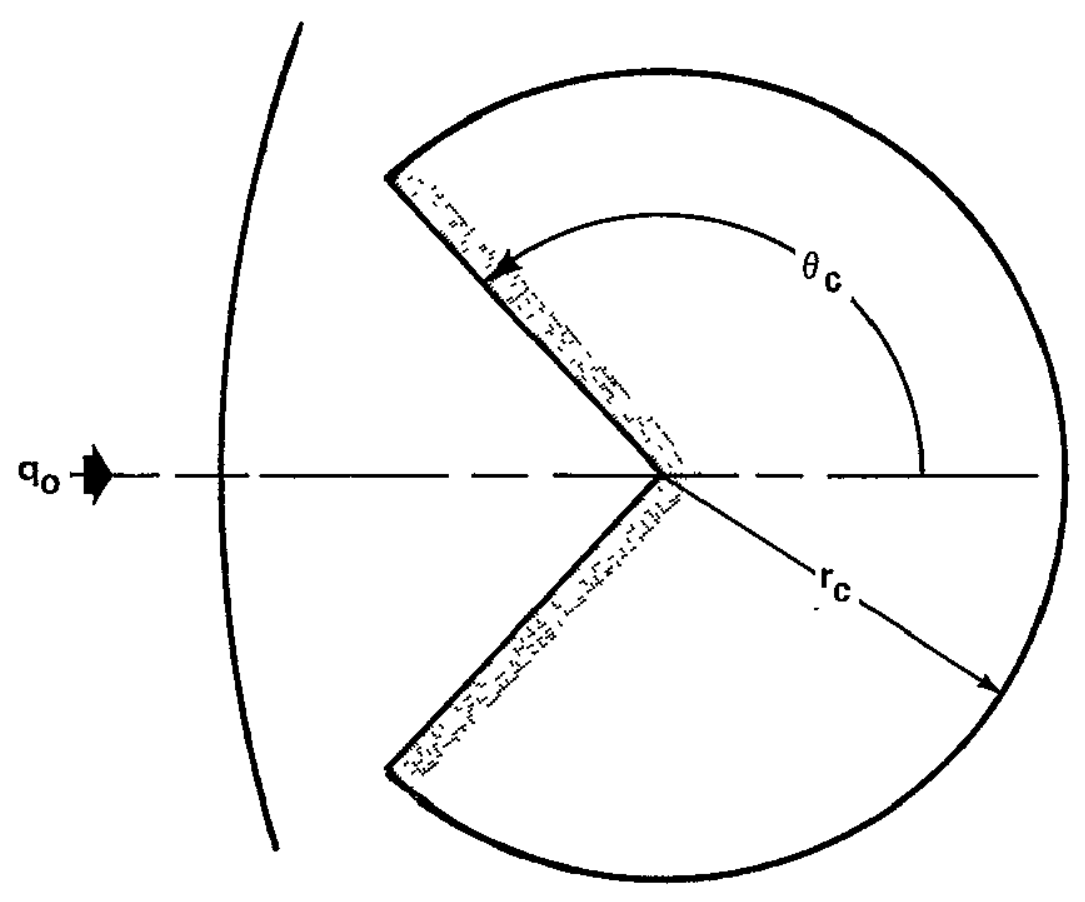

c 


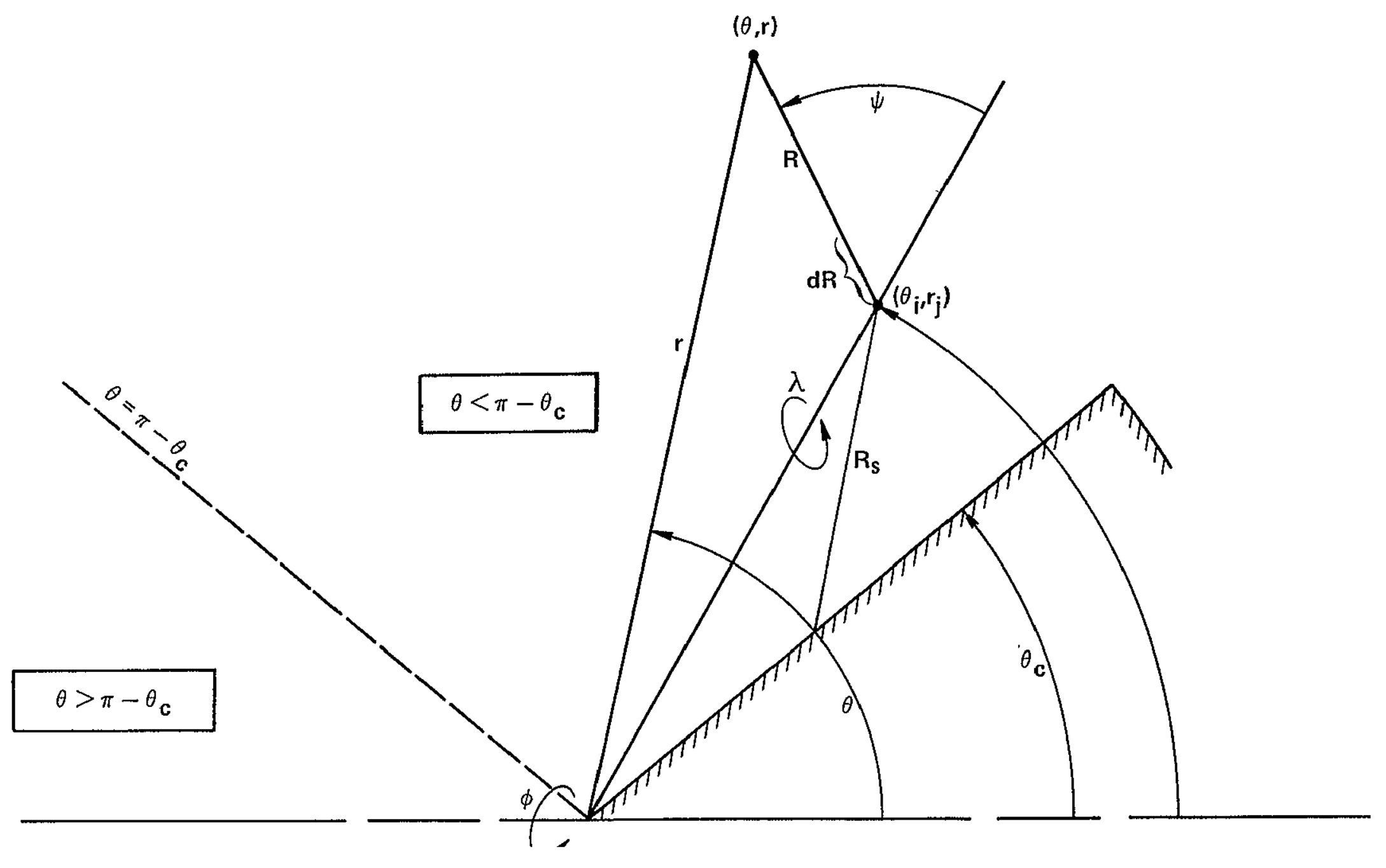

FIGURE 2 - SPHERICAL COORDINATE SYSTEMS 


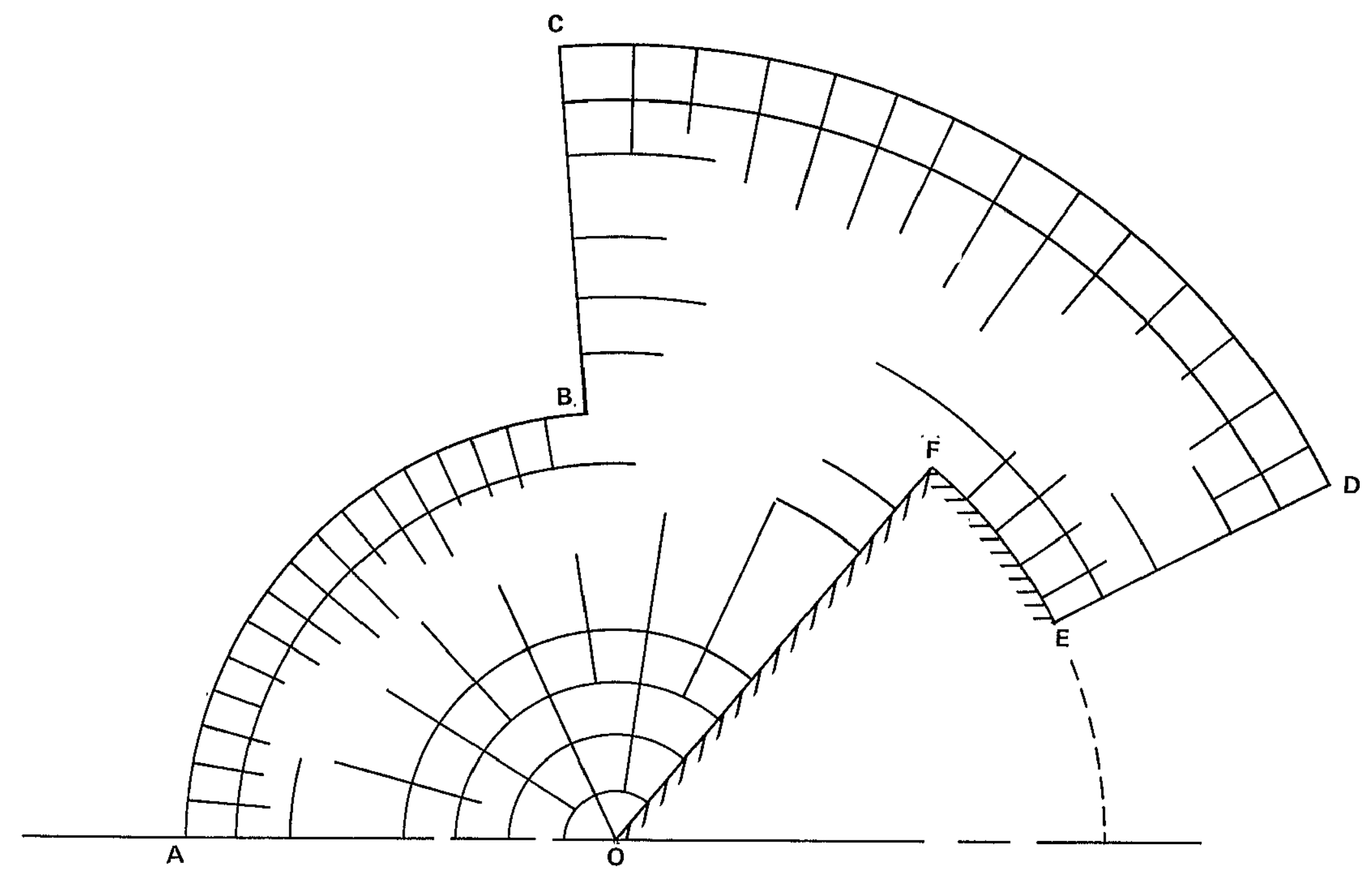

FIGURE 3 - NONUNIFORM COMPUTATIONAL GRID 


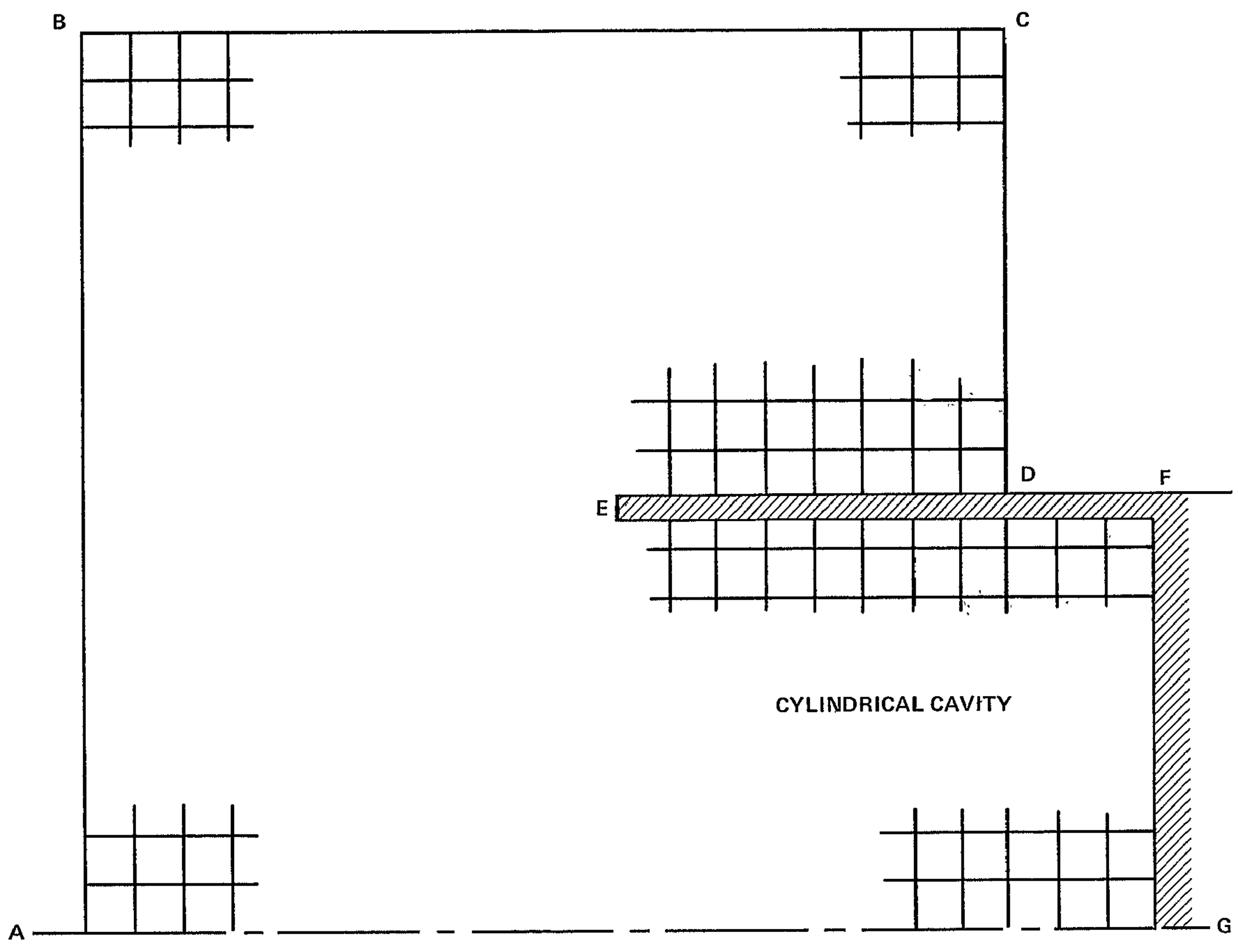

FIGURE 4 - COMPUTATIONAL GRID FOR CYLINDER 


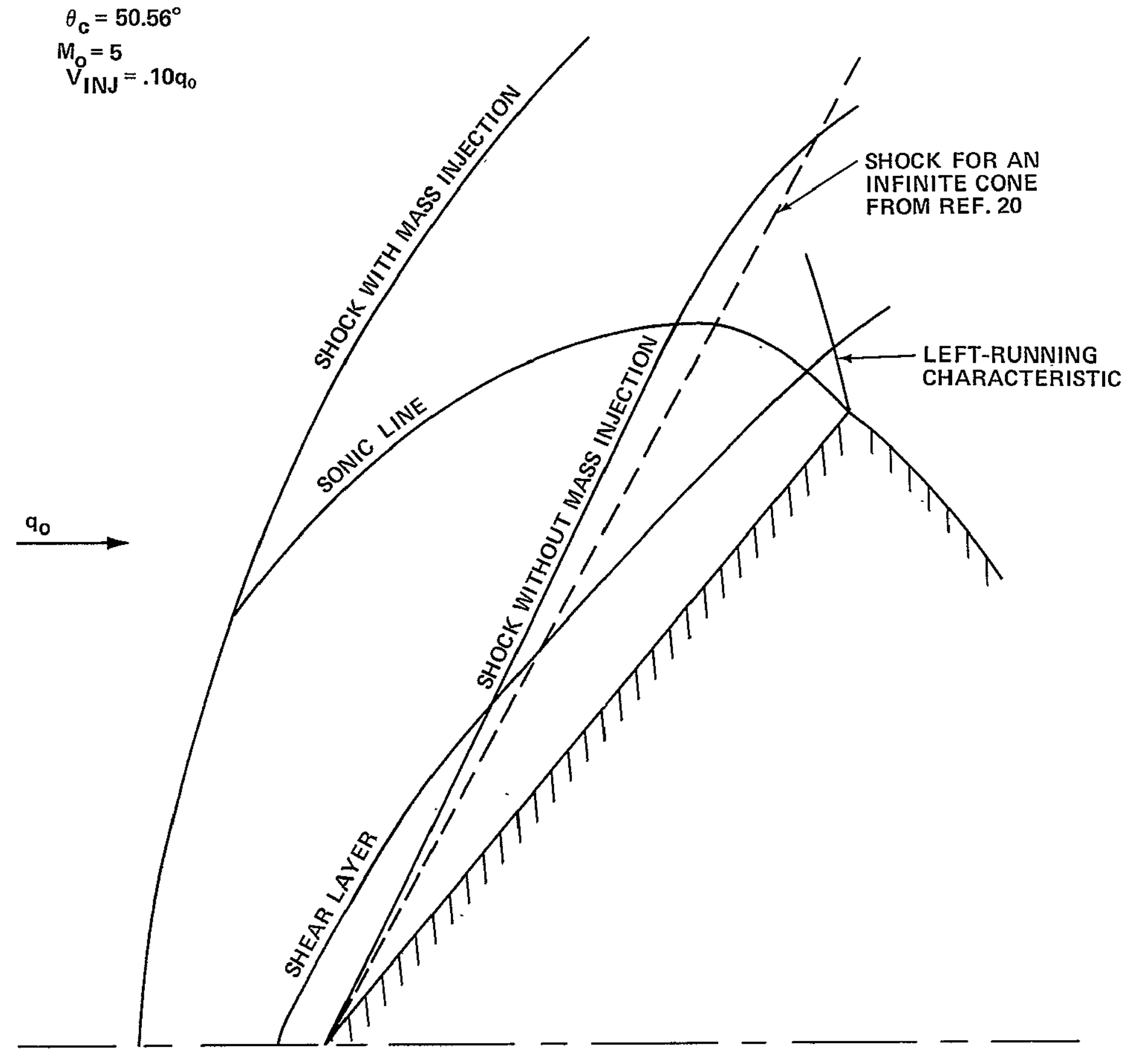

FIGURE 5 - FLOW OVER A $50.56^{\circ}$ HALF-ANGLE CONE 


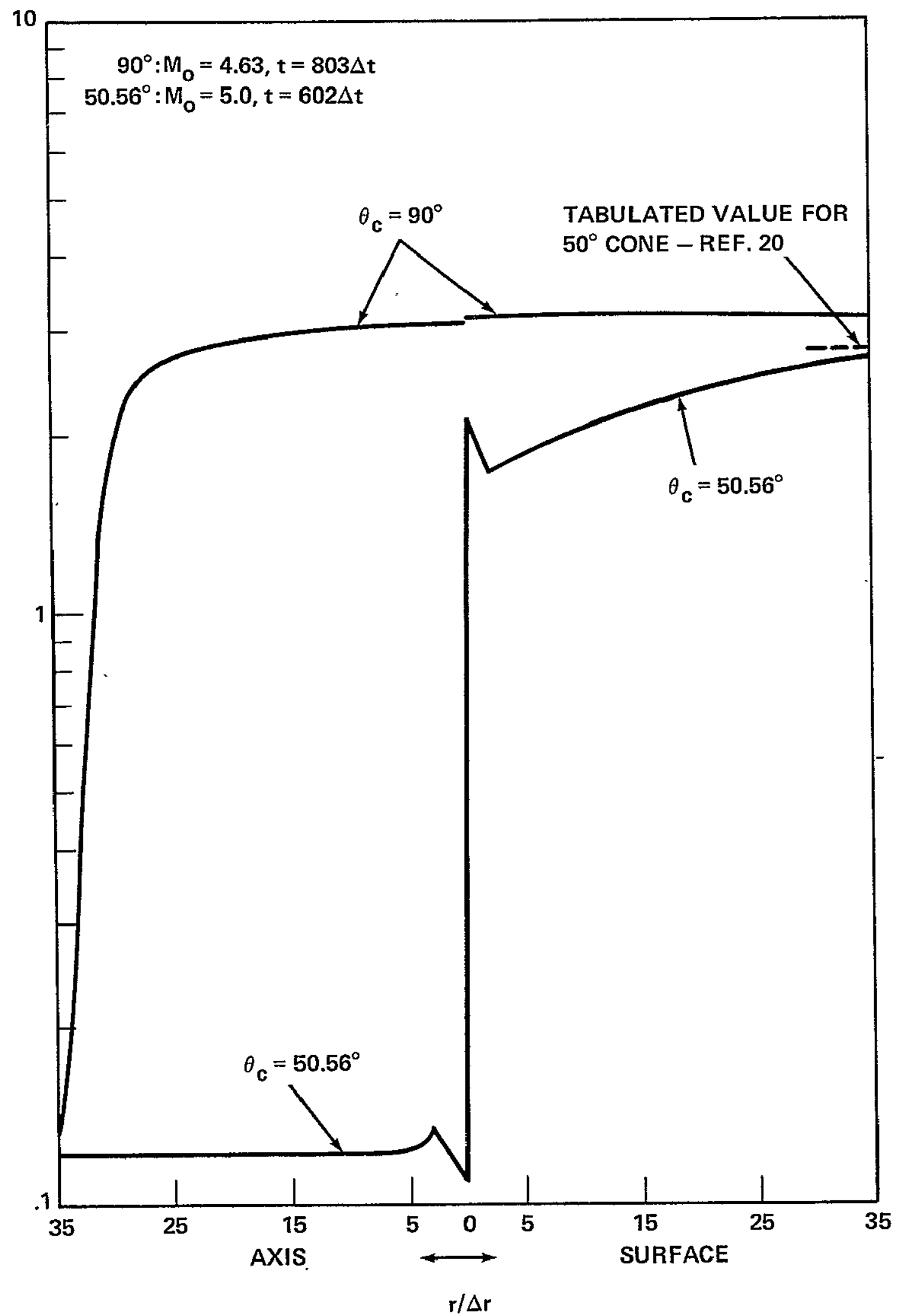

FIGURE 6 - PRESSURE ALONG AXIS-SURFACE STREAMLINE FOR $50.56^{\circ}$ AND $90^{\circ}$ HALF ANGLE CONES 


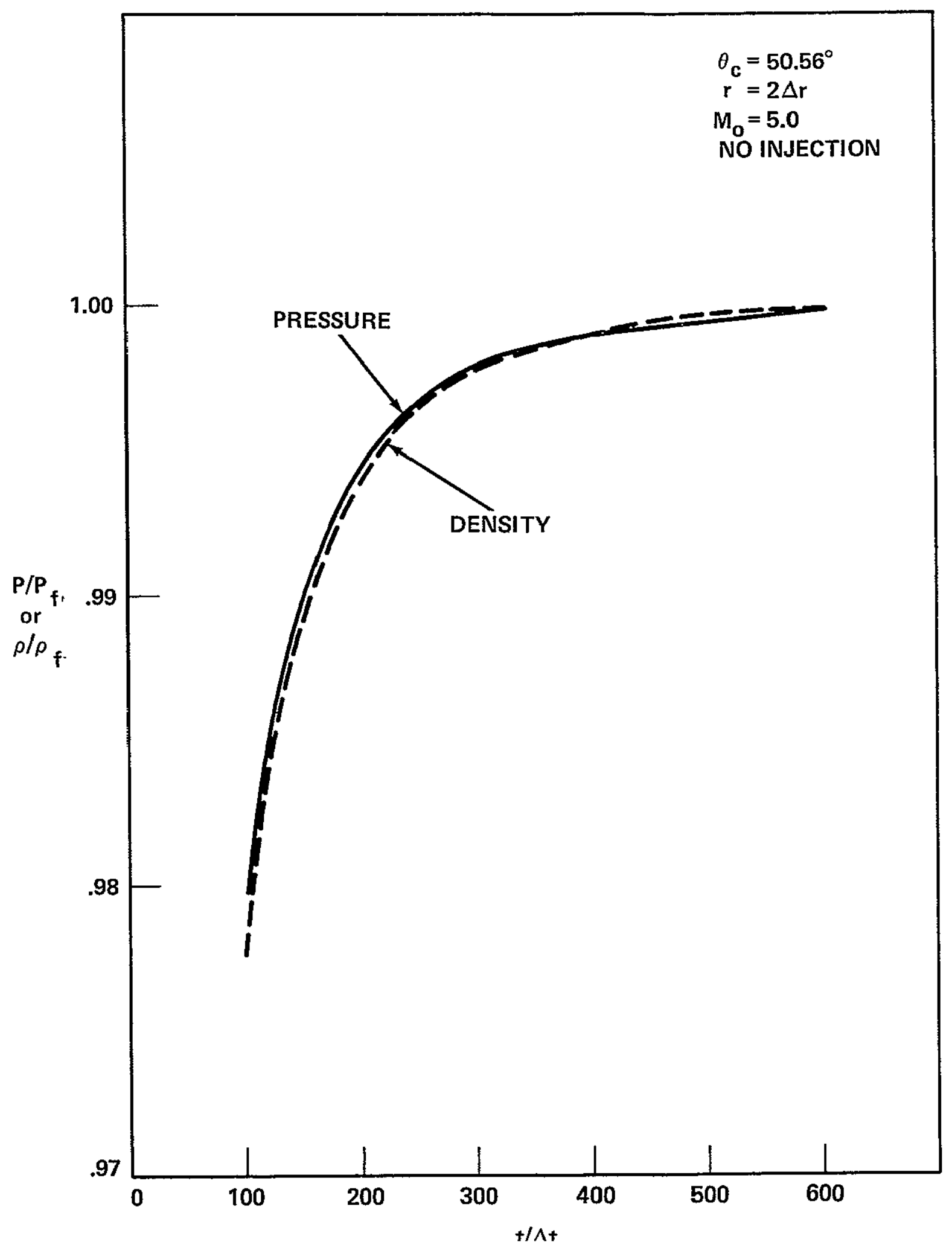

FIGURE 7 - PRESSURE AND DENSITY TRANSIENTS NEAR VERTEX FOR $50.56^{\circ}$ CONE 


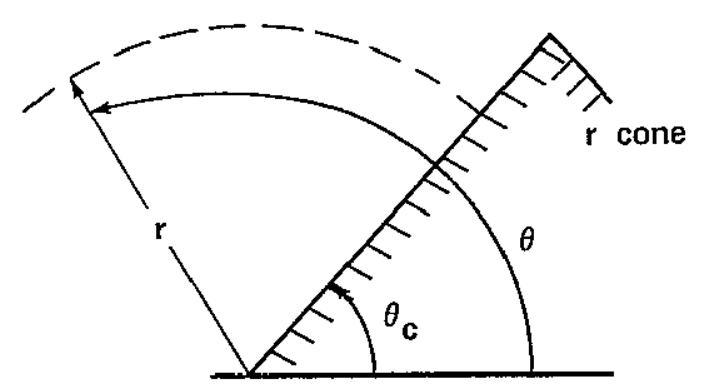

$$
\begin{aligned}
M_{o} & =5 \\
r & =50 \Delta r \\
r \text { cone } & =65 \Delta r
\end{aligned}
$$

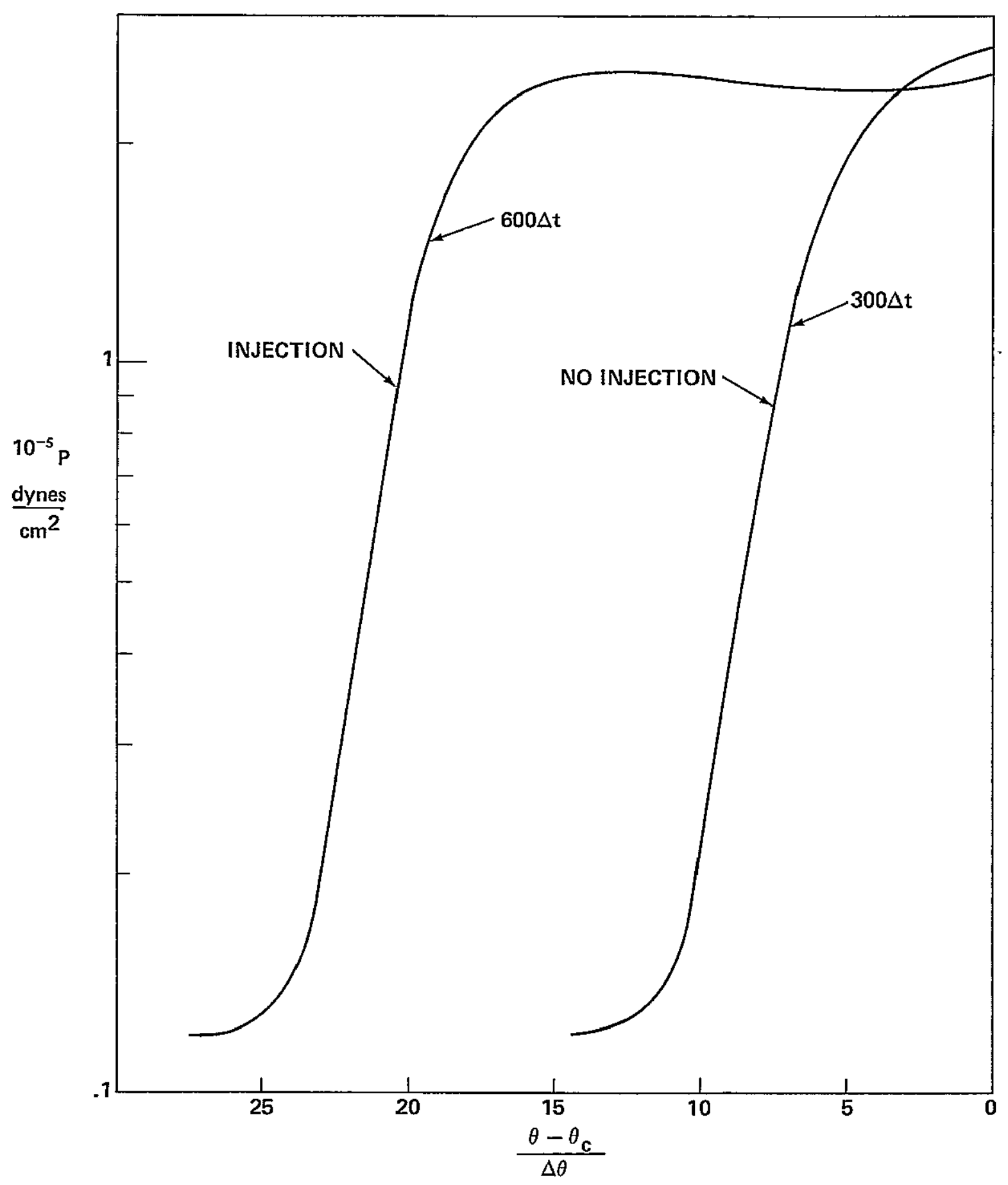

FIGURE 8 - PRESSURE PROFILES IN SHOCK LAYER 


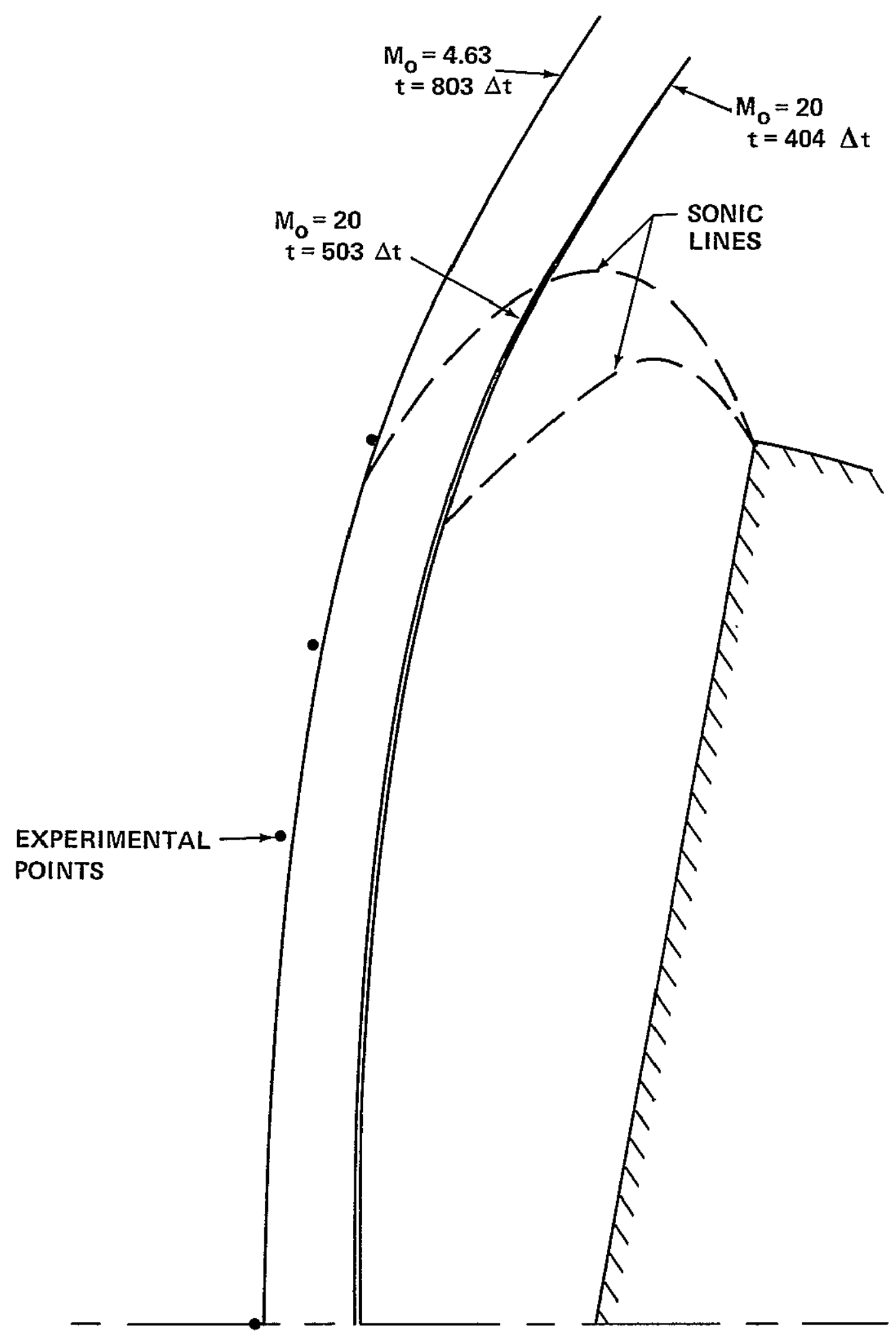

FIGURE 9 - SHOCK SHAPES FOR A $79.83^{\circ}$ HALF ANGLE CONE 


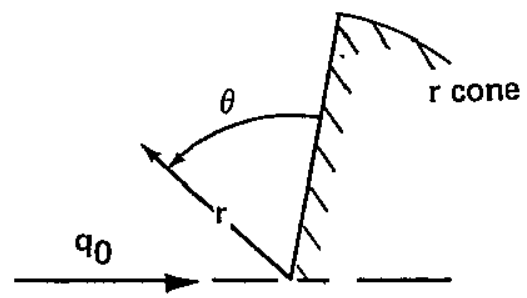

$$
\begin{aligned}
& \theta_{c}=79.83^{\circ} \\
& M_{o}=20 . \\
& r=36 \Delta r \\
& r \text { cone }=65 \Delta r
\end{aligned}
$$

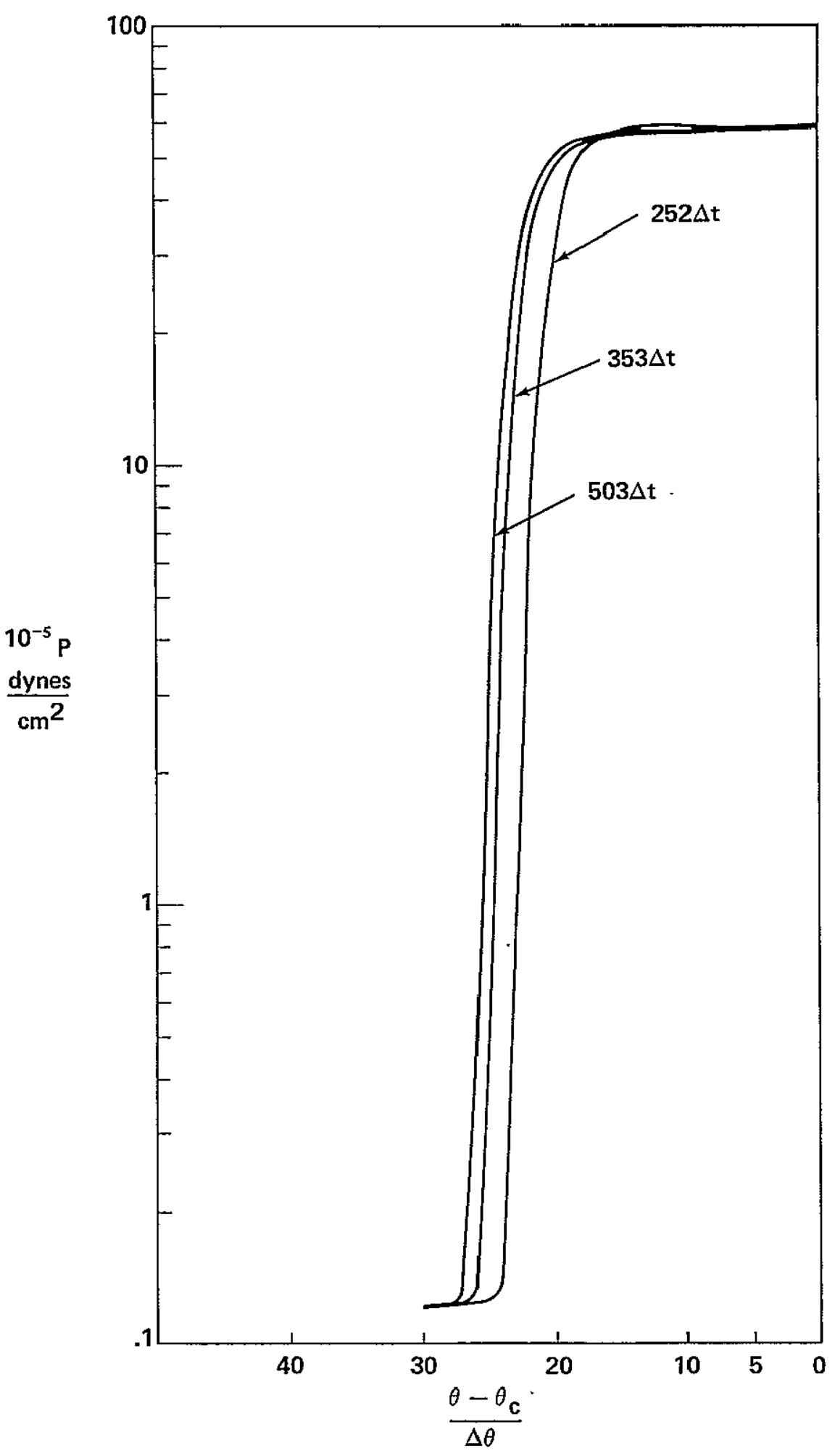

FIGURE.10 - HISTTORY OF PRESSURE DISTRIBUTION IN SHOCK LAYER 

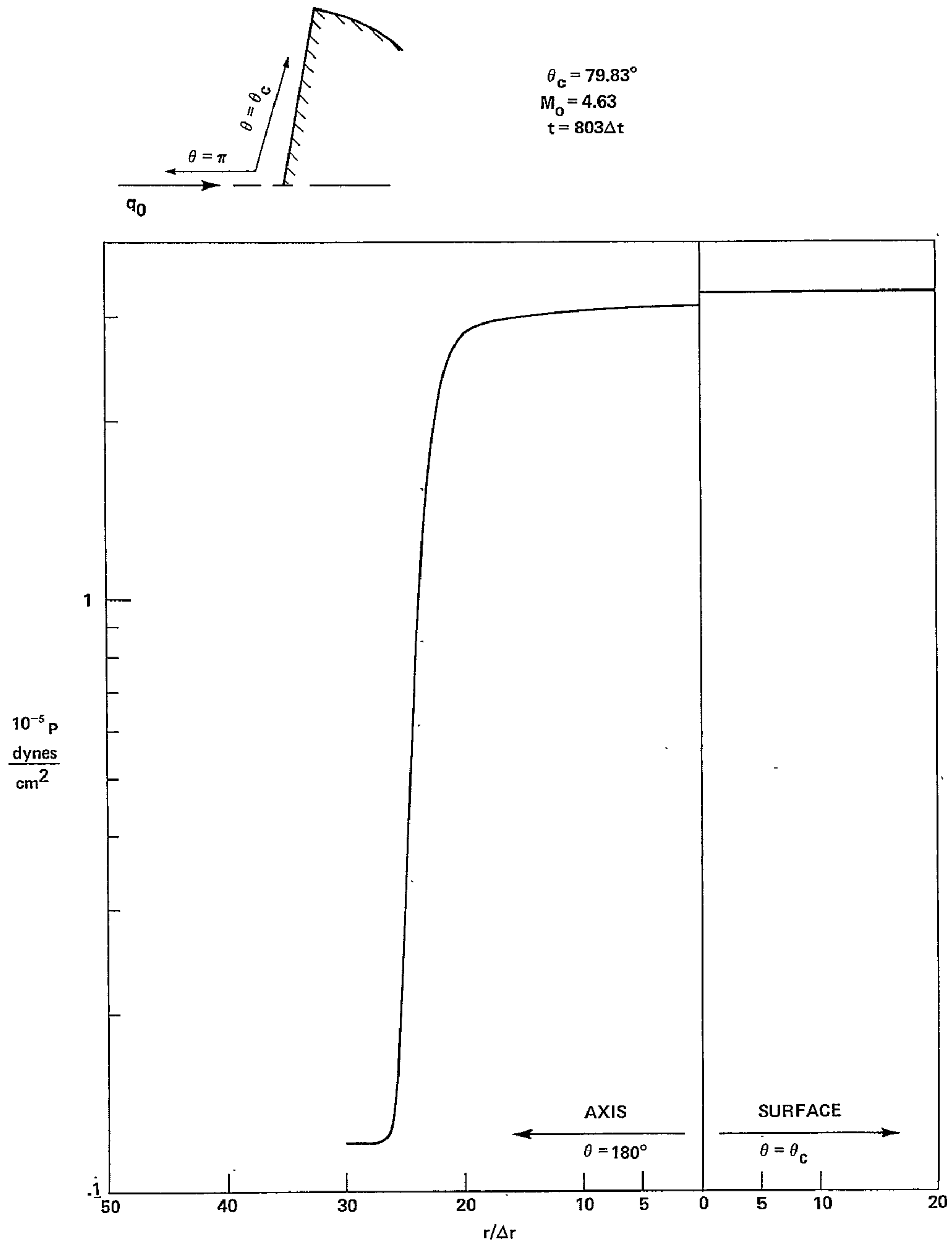

FIGURE'11 - PRESSURE ALONG AXIS-SURFACE STREAMLINE 

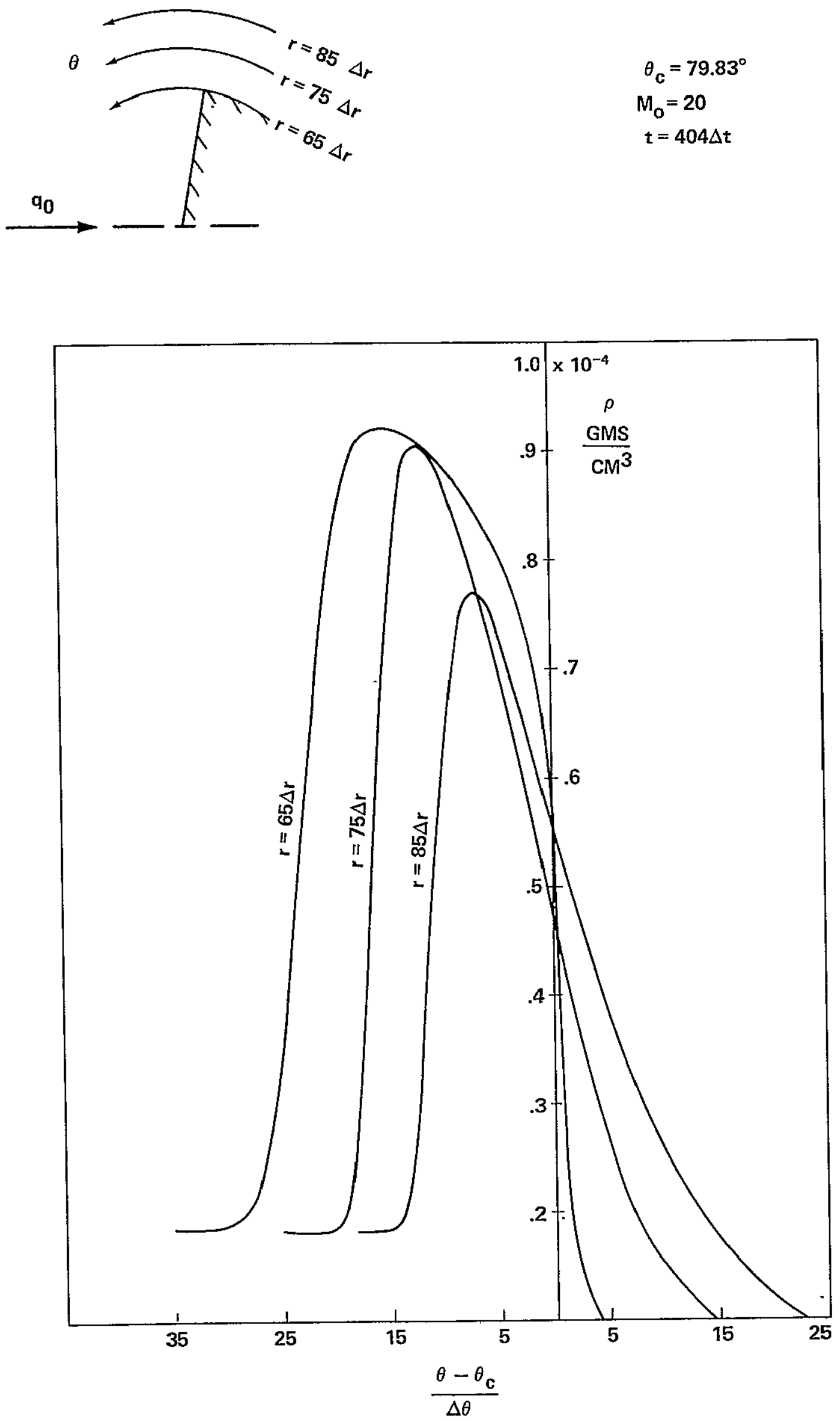

FIGURE 12 - DENSITY VS. ANGLE BEHIND CONE 


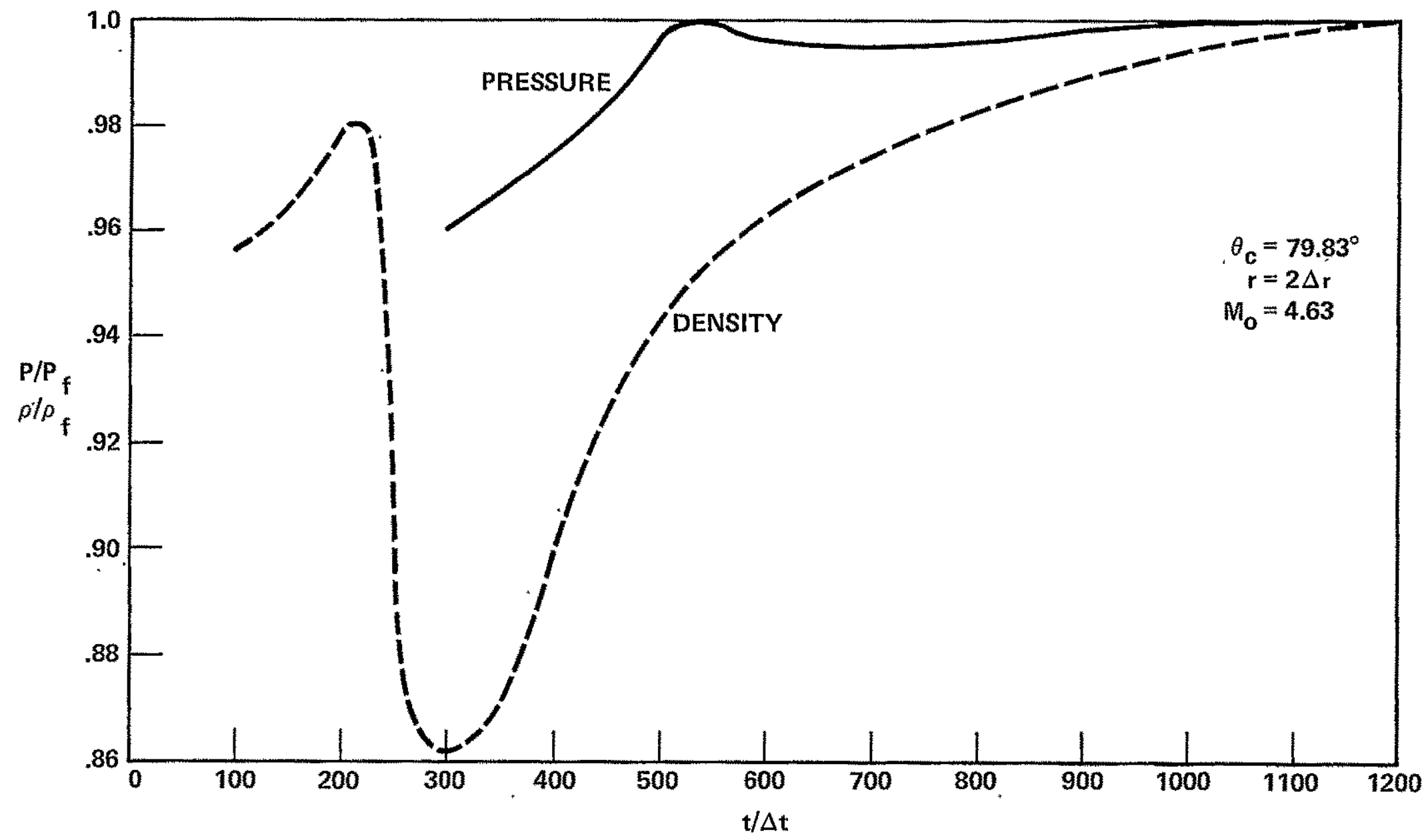

FIGURE 13 - PRESSURE AND DENSITY TRANSIENTS NEAR VERTEX FOR $79.83^{\circ}$ CONE 

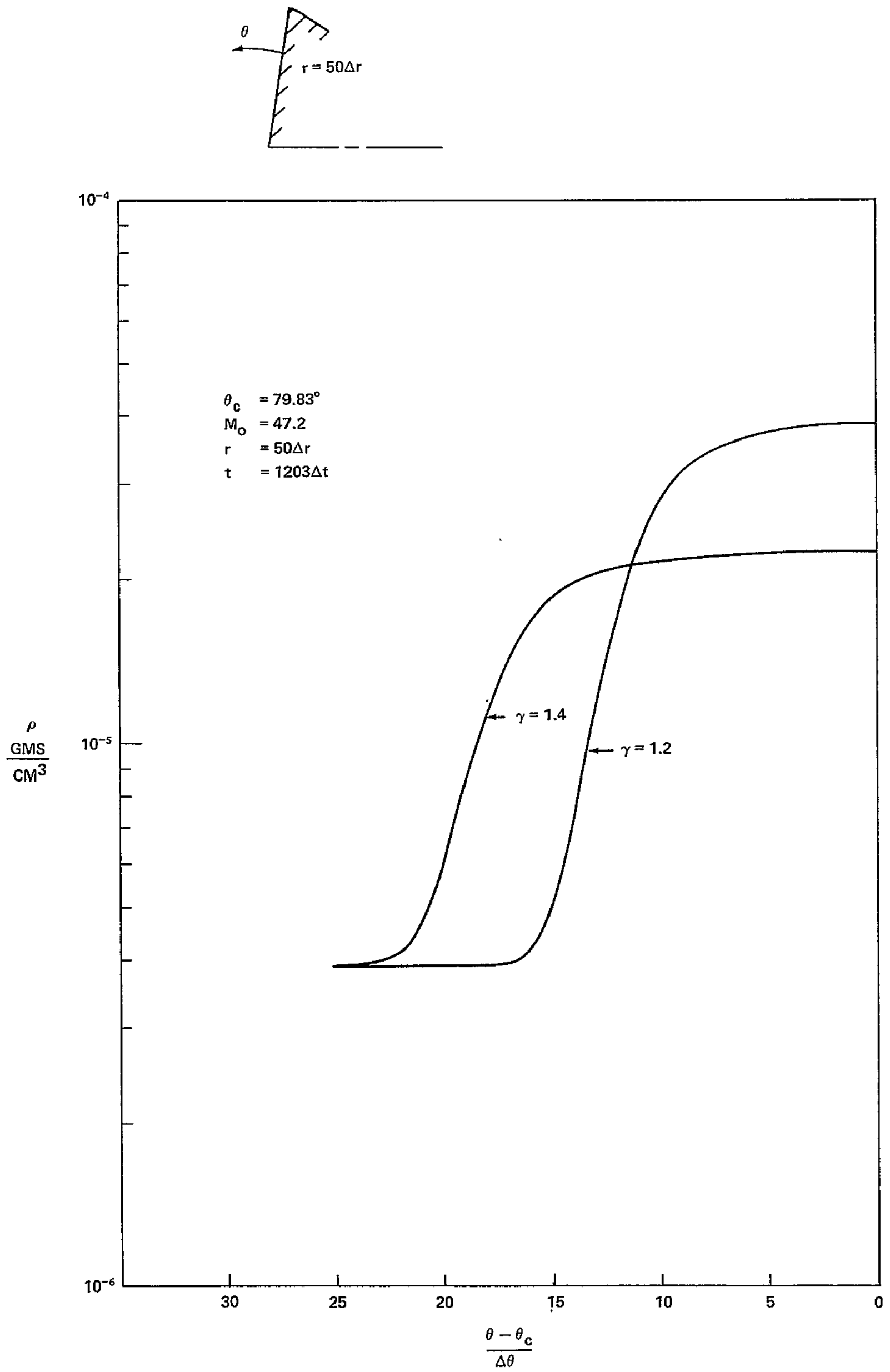

FIGURE 14 - DENSITY PROFILE IN SHOCK LAYER 

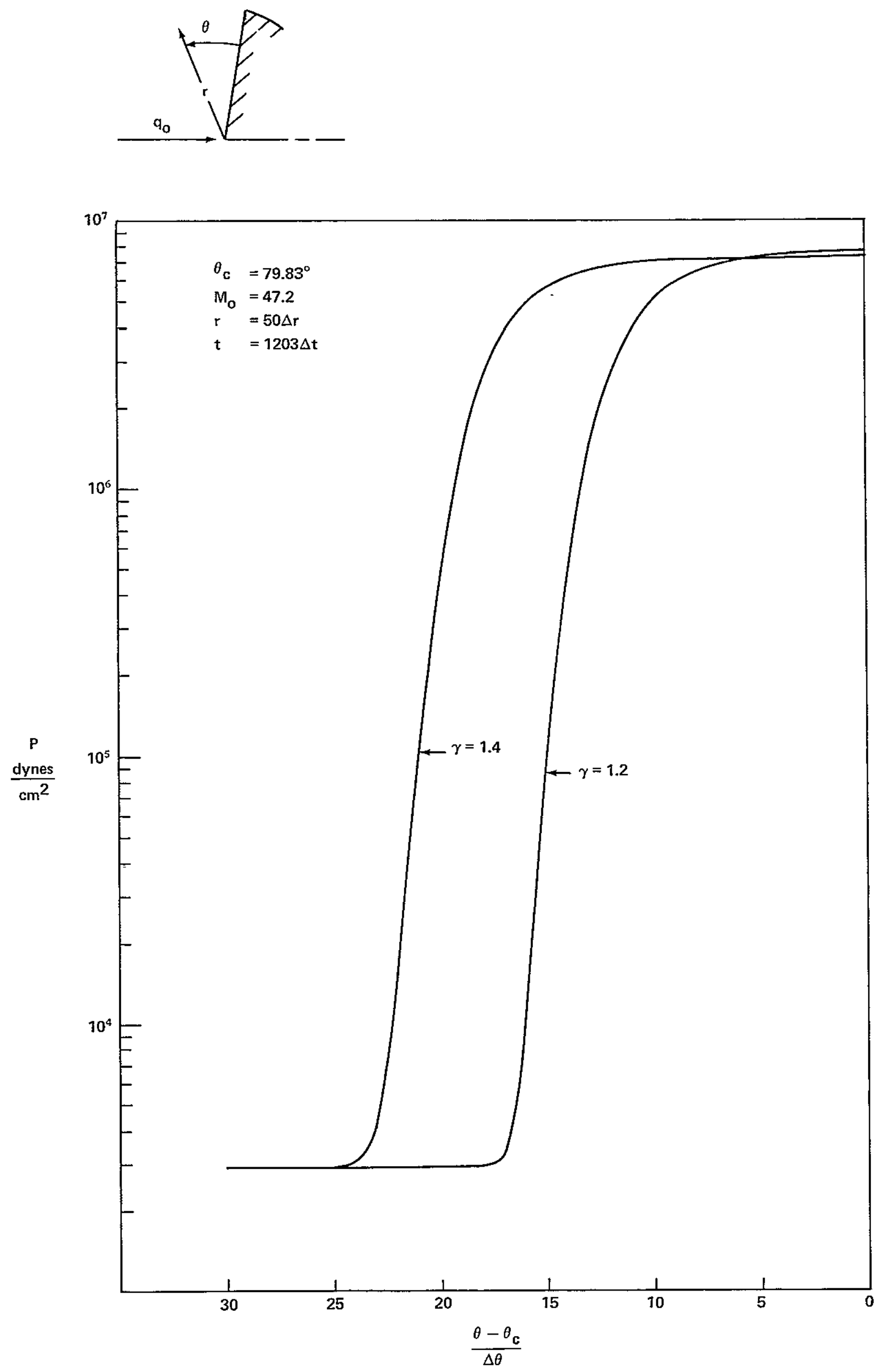

FIGURE 15 - PRESSURE PROFILE IN SHOCK LAYER 


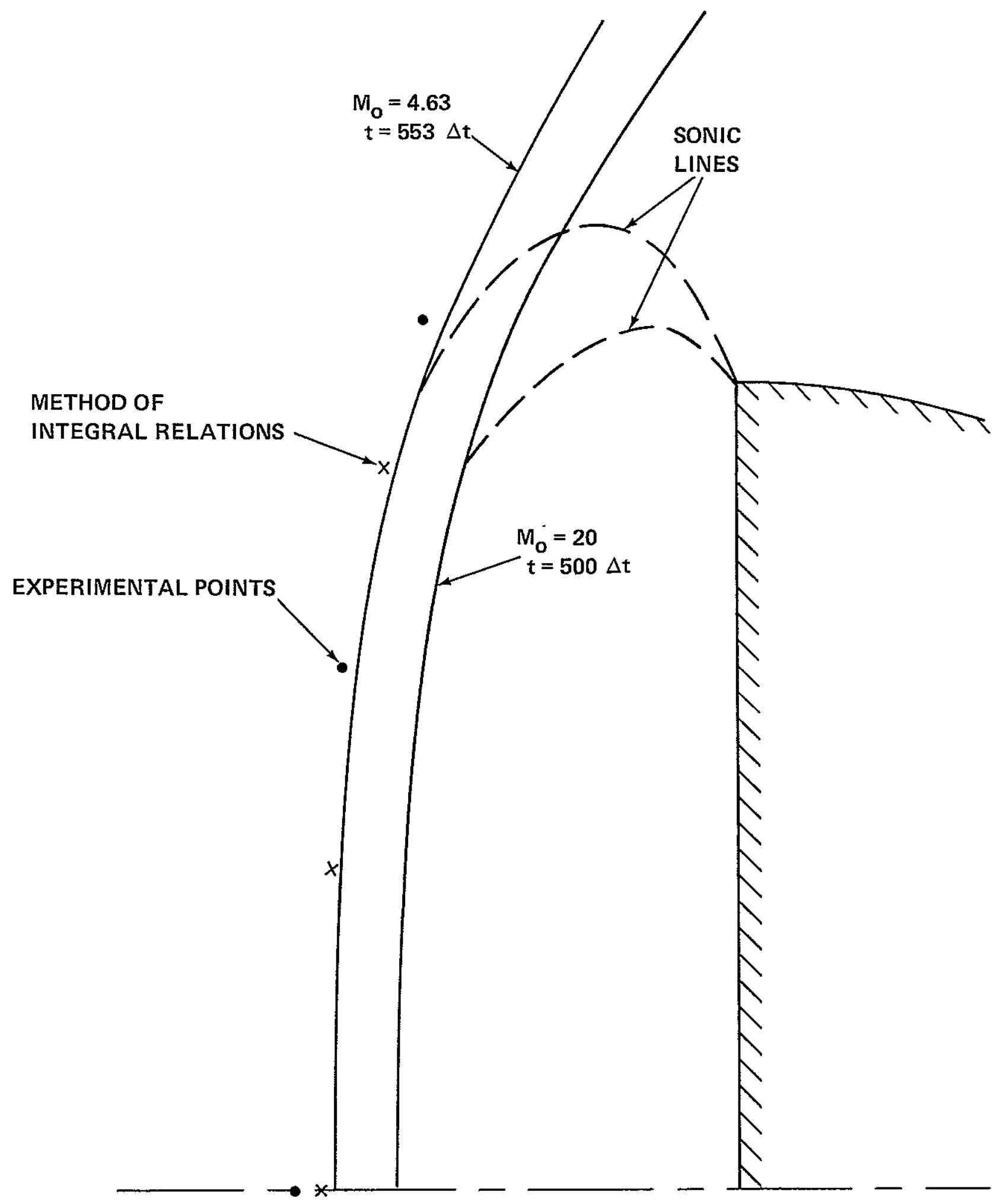

FIGURE 16 - SHOCK SHAPES FOR A $90^{\circ}$ HALF ANGLE CONE 


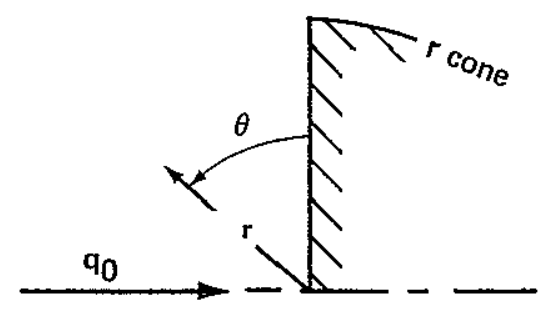

$$
\begin{gathered}
\theta_{c}=90^{\circ} \\
M_{o}=4.63 \\
r=36 \Delta r
\end{gathered}
$$

$r$ cone $=65 \Delta r$

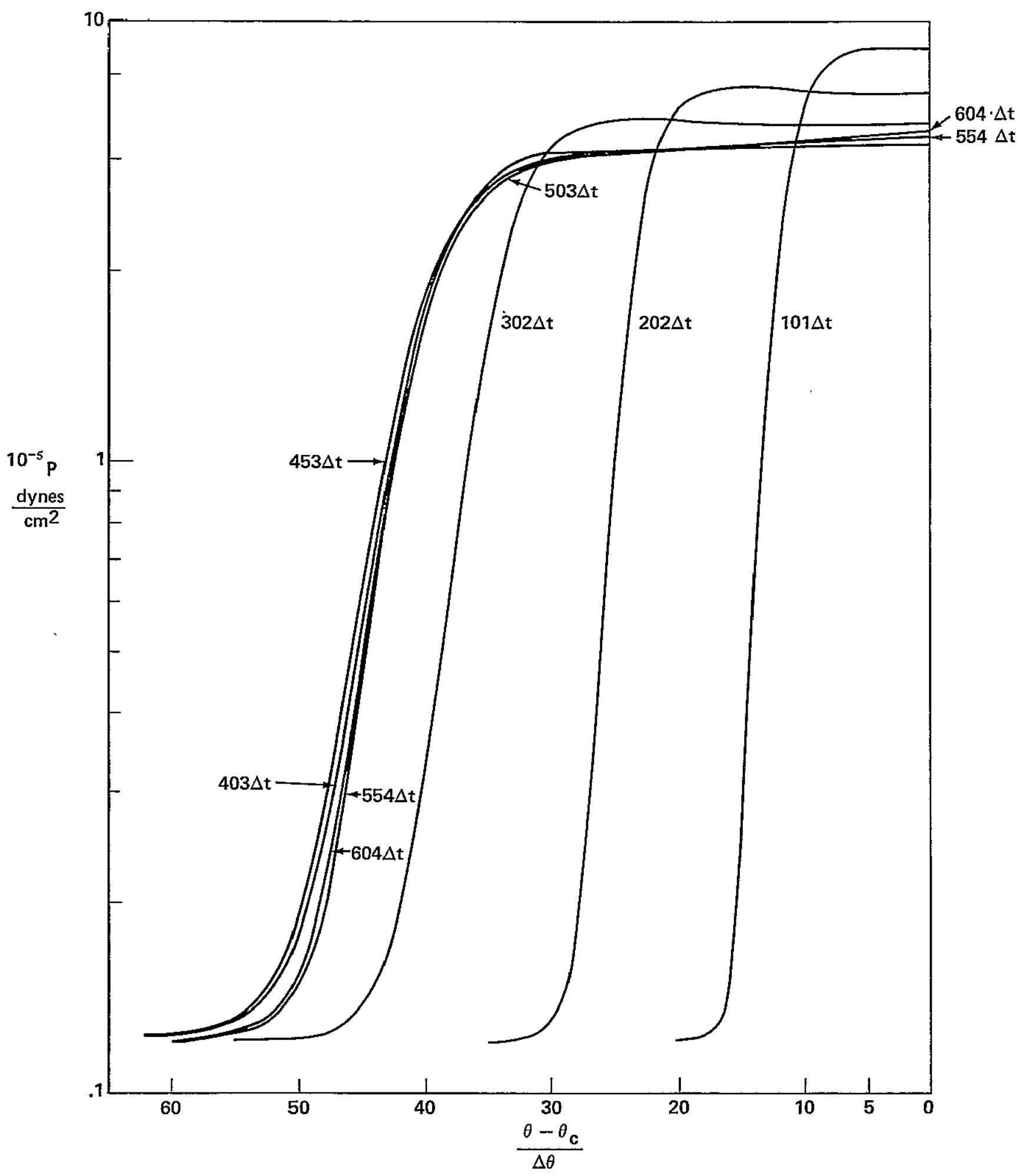

FIGURE 17 - HISTORY OF PREṠSURE DISTRIBUTION IN SHOCK LAYER 


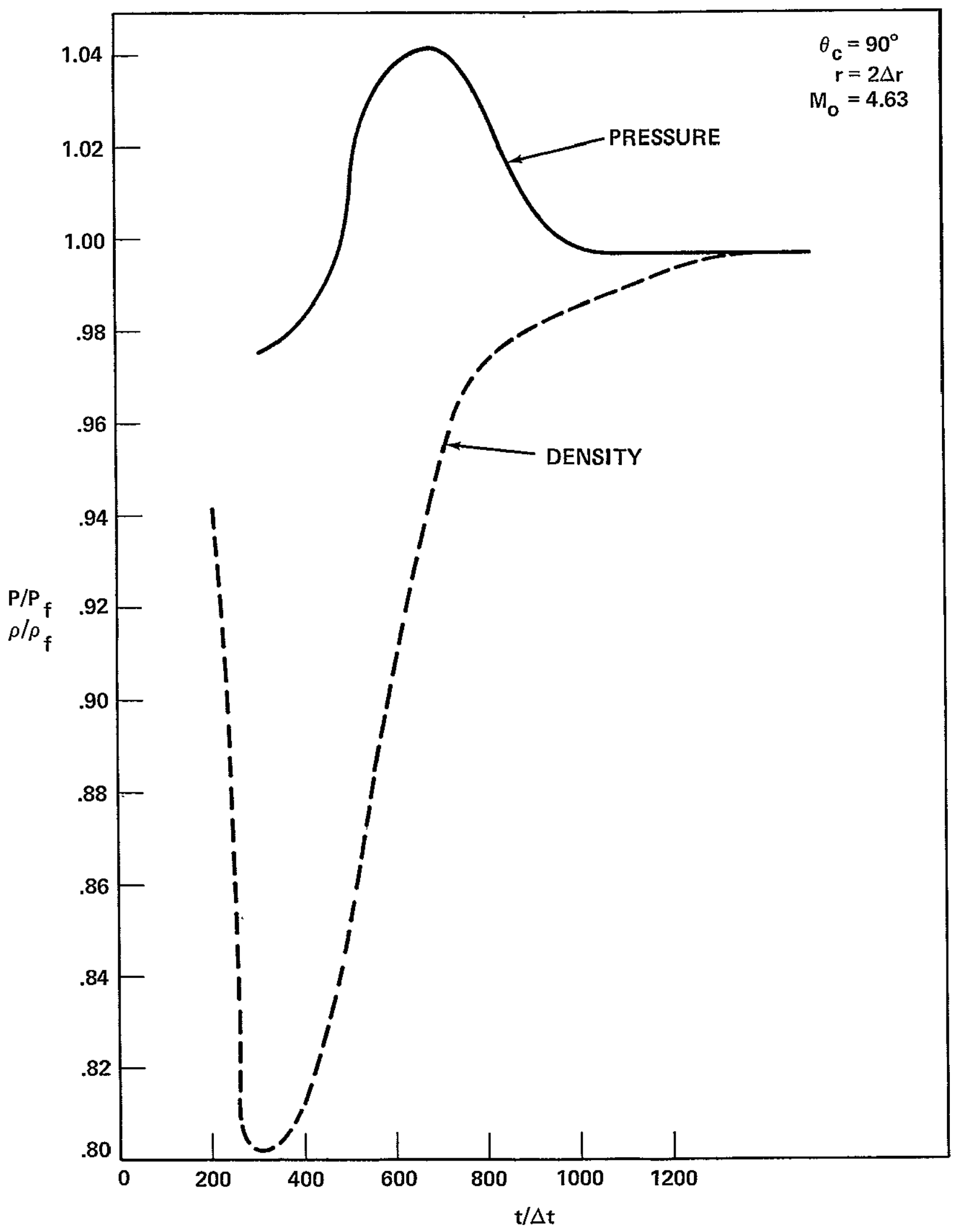

FIGURE 18 - PRESSURE AND DENSITY TRANSIENTS NEAR VERTEX FOR $90^{\circ}$ CONE 


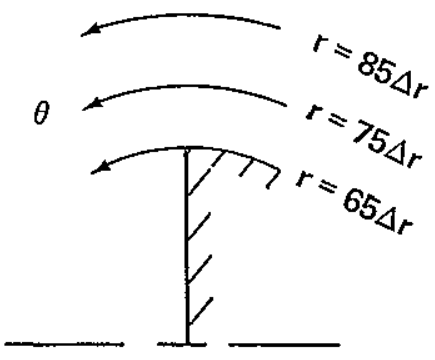

$$
\begin{gathered}
\theta_{c}=90^{\circ} \\
M_{o}=4.63 \\
t=706 \Delta t
\end{gathered}
$$

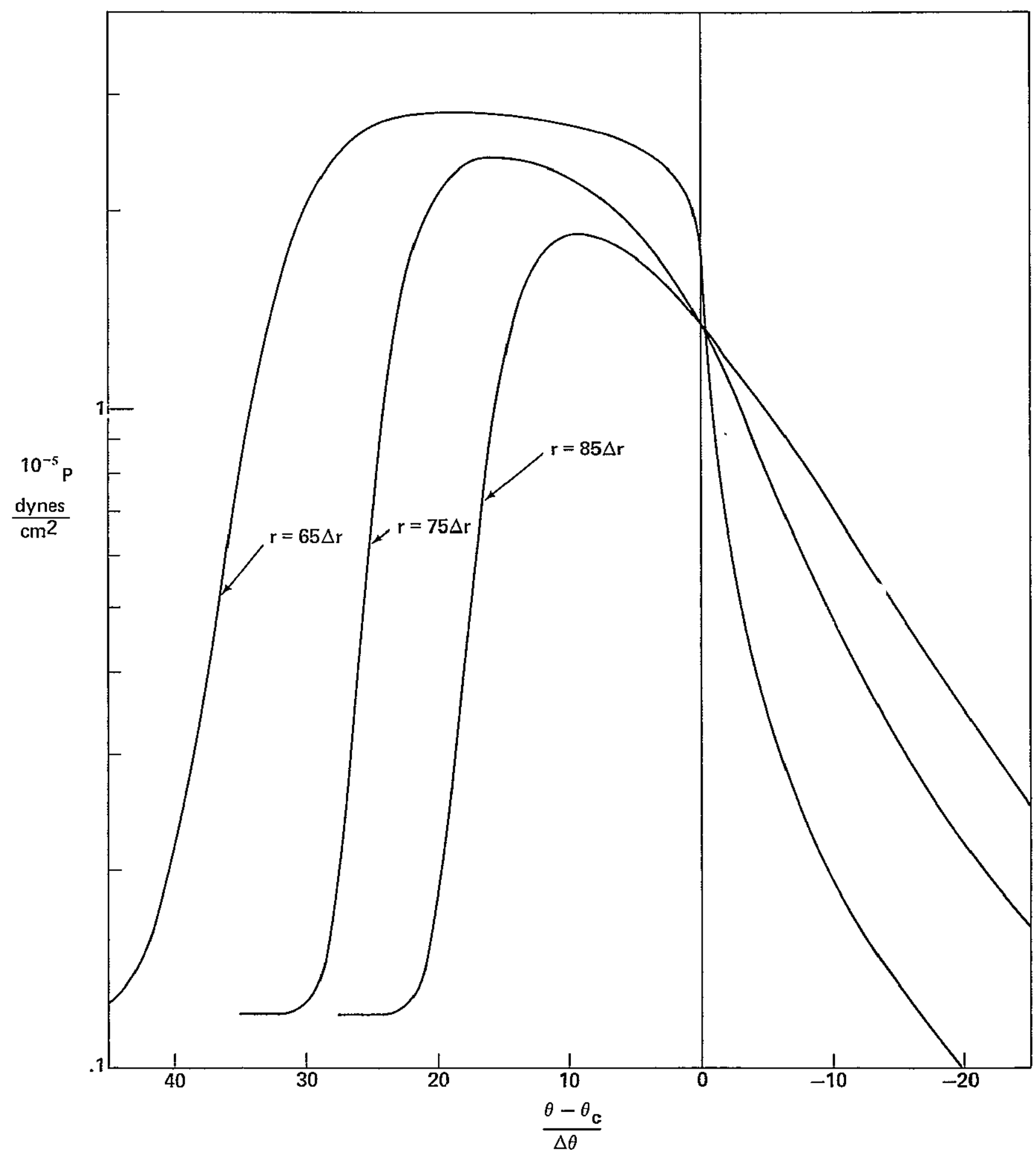

FIGURE 19 - PRESSURE VS. ANGLE BEHIND CONE 


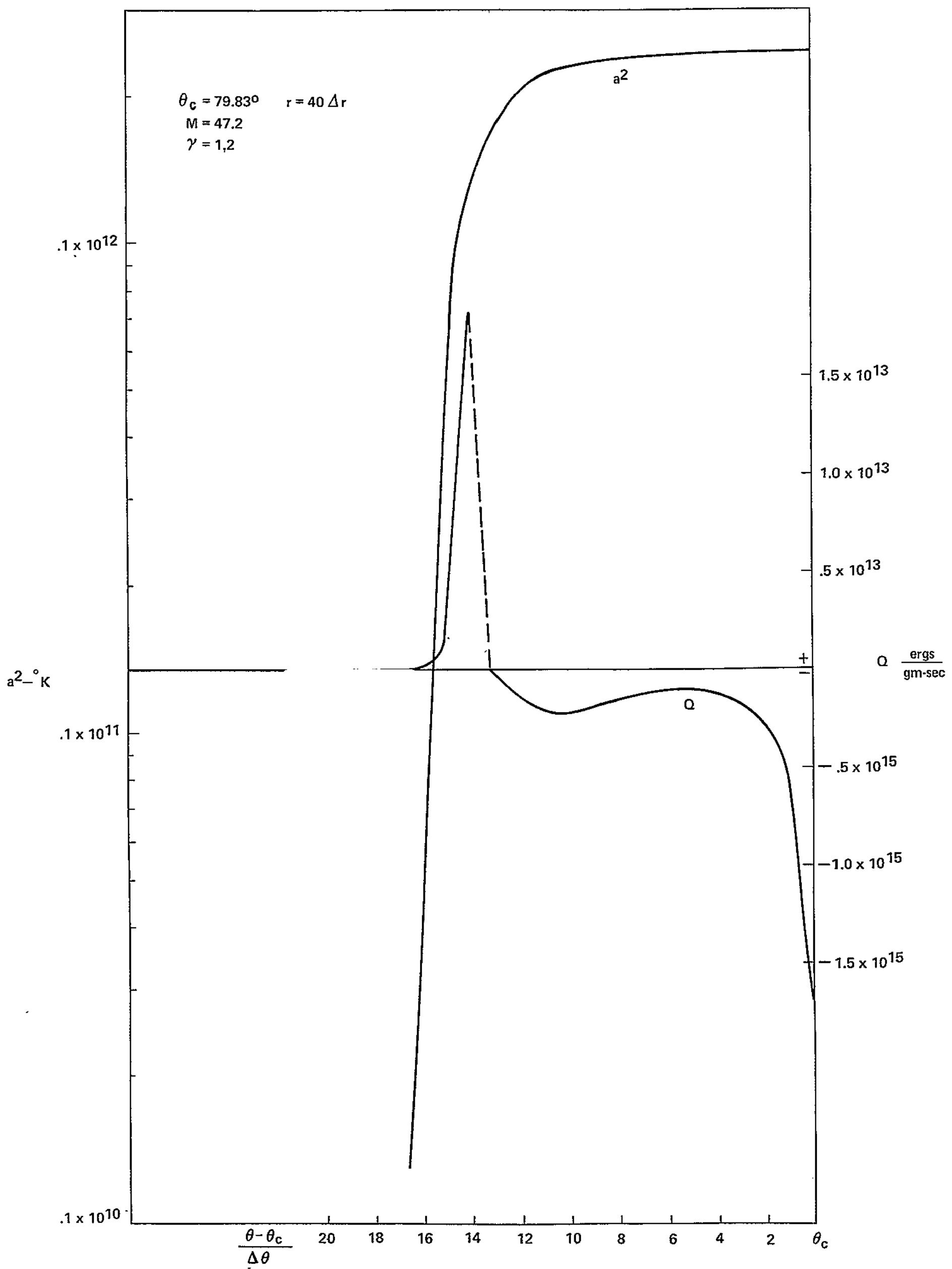

FIGURE 20 - TEMPERATURE AND RADIATIVE ENERGY GAIN 


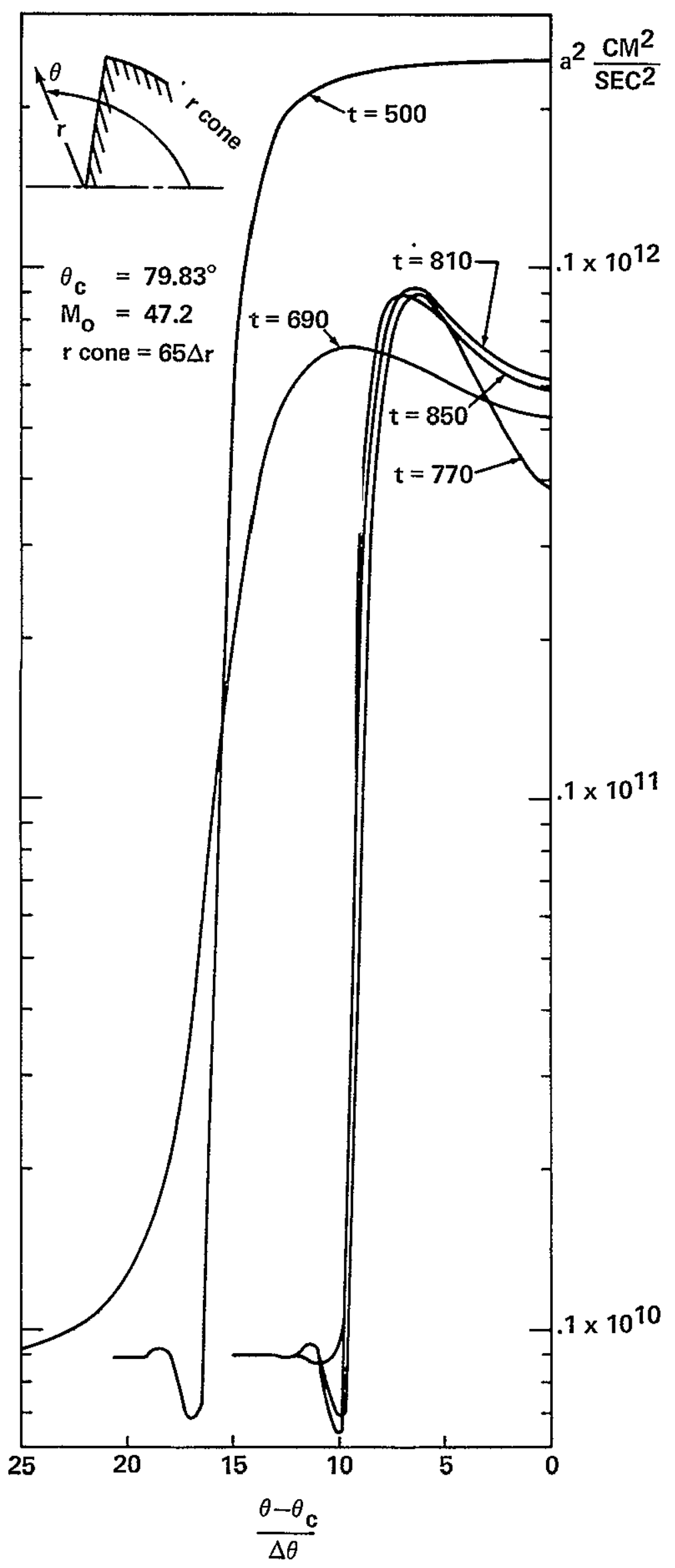

FIGURE 21 - EFFECT OF RADIATION ON THE SHOCK LAYER 


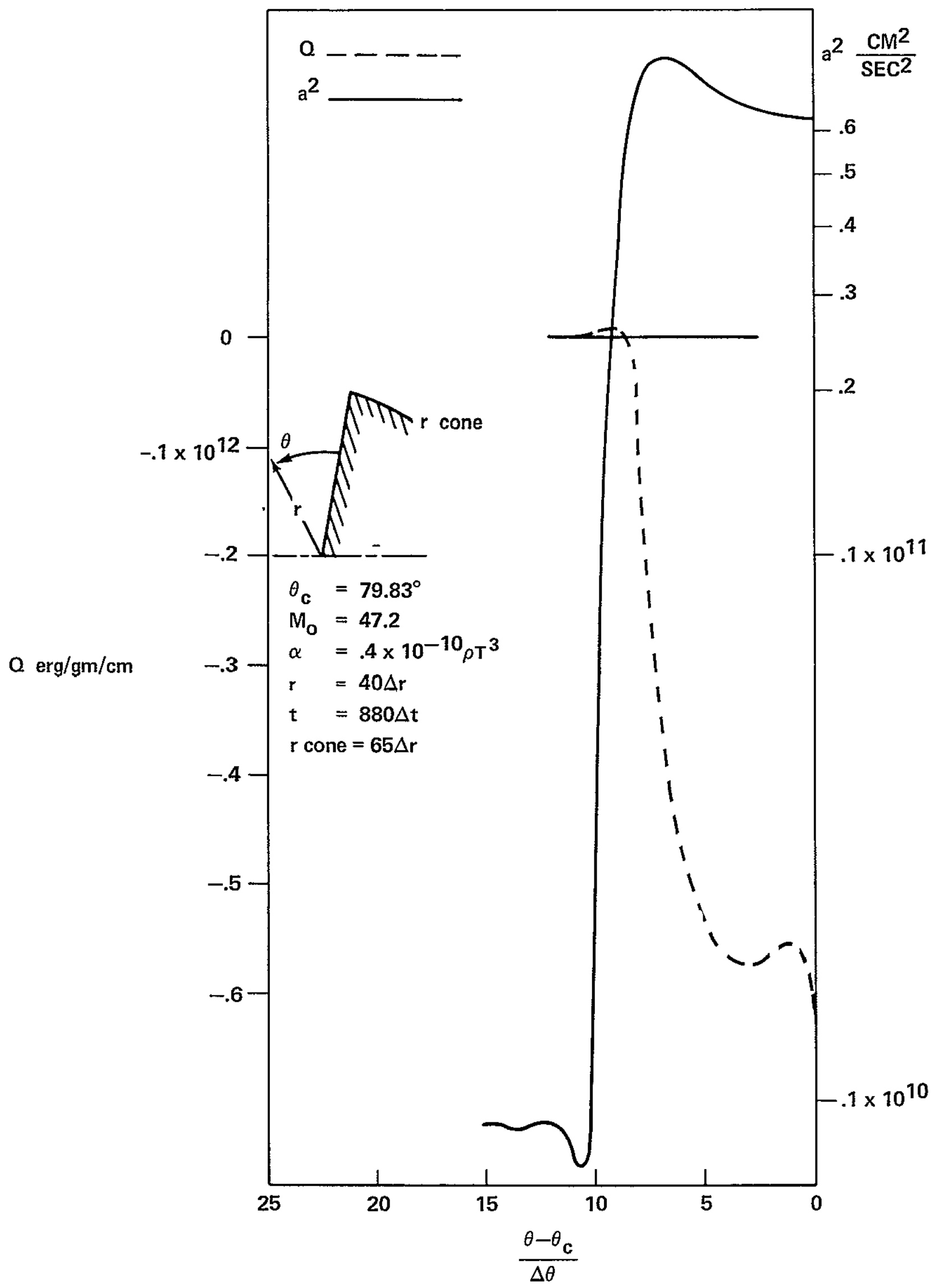

FIGURE 22 - SHOCK LAYER PROFILE WITH RADIATION EFFECTS 


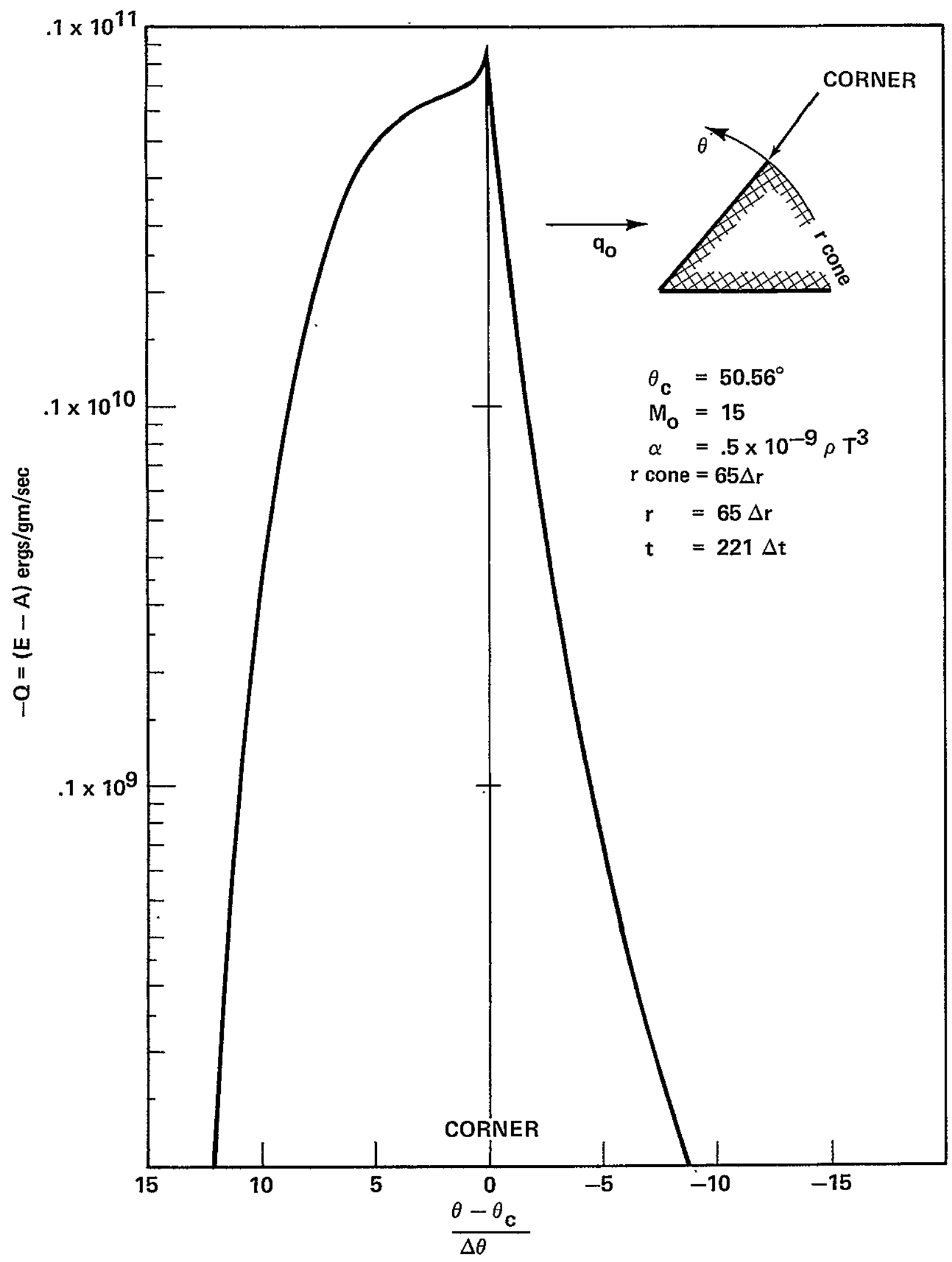

FIGURE 23 - SHOCK LAYER EMISSION 


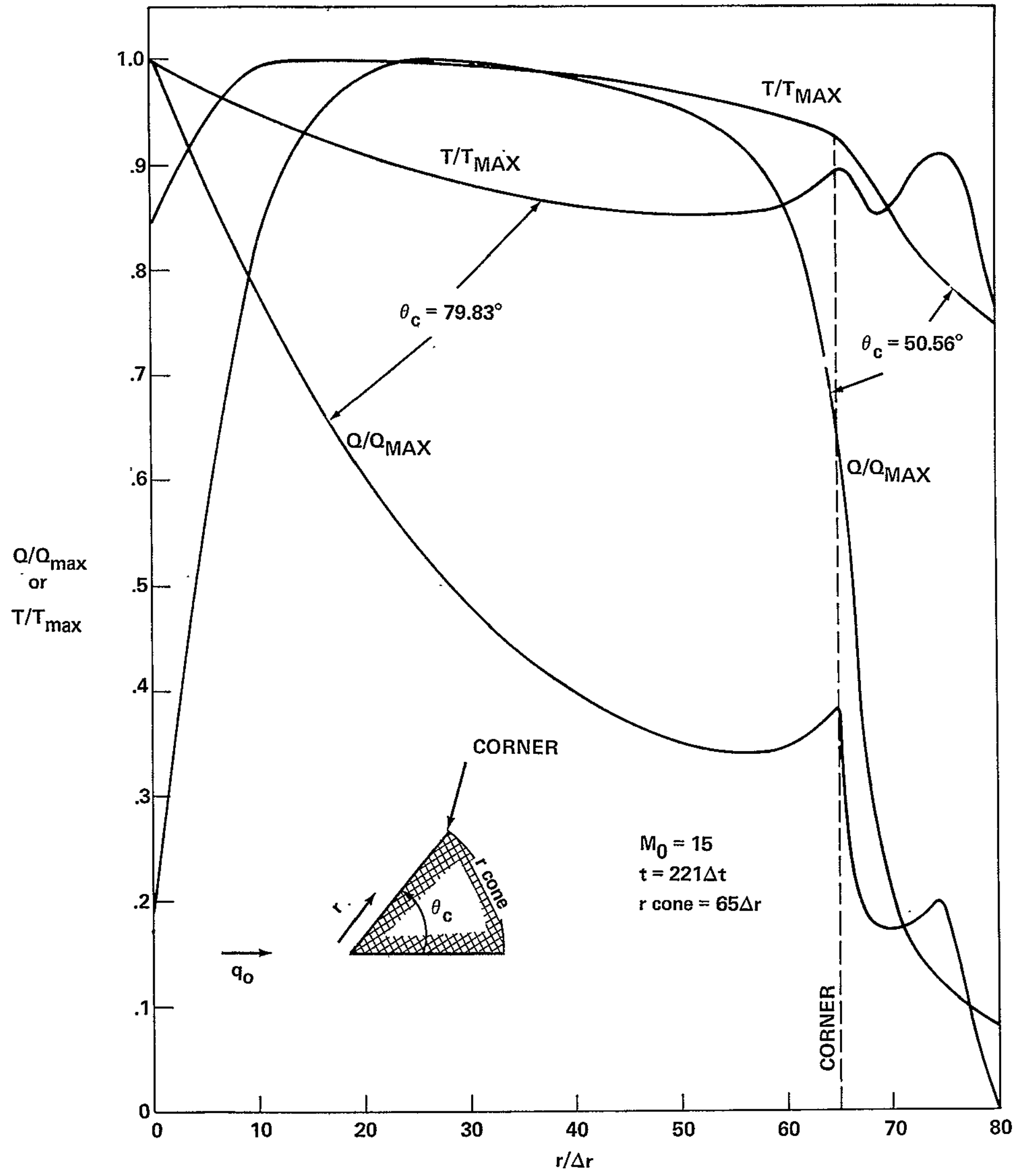

FIGURE 24 - TEMPERATURE AND EMISSION ALONG THE SURFACE 

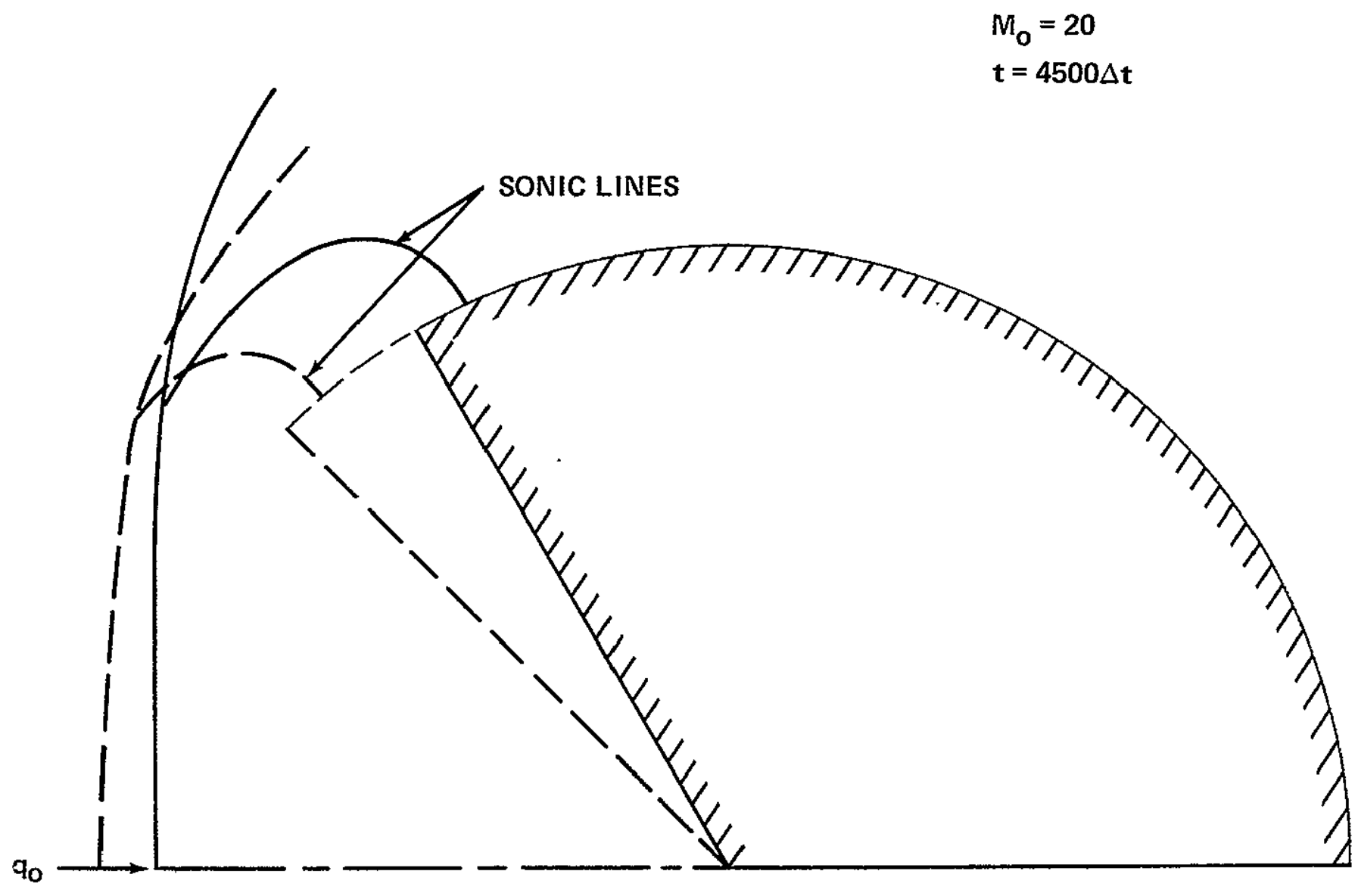

$120^{\circ}$ CONE

$135^{\circ}$ CONE

FIGURE 25 - SHOCK SHAPES FOR $120^{\circ}$ AND $135^{\circ}$ HALF ANGLE CONES 


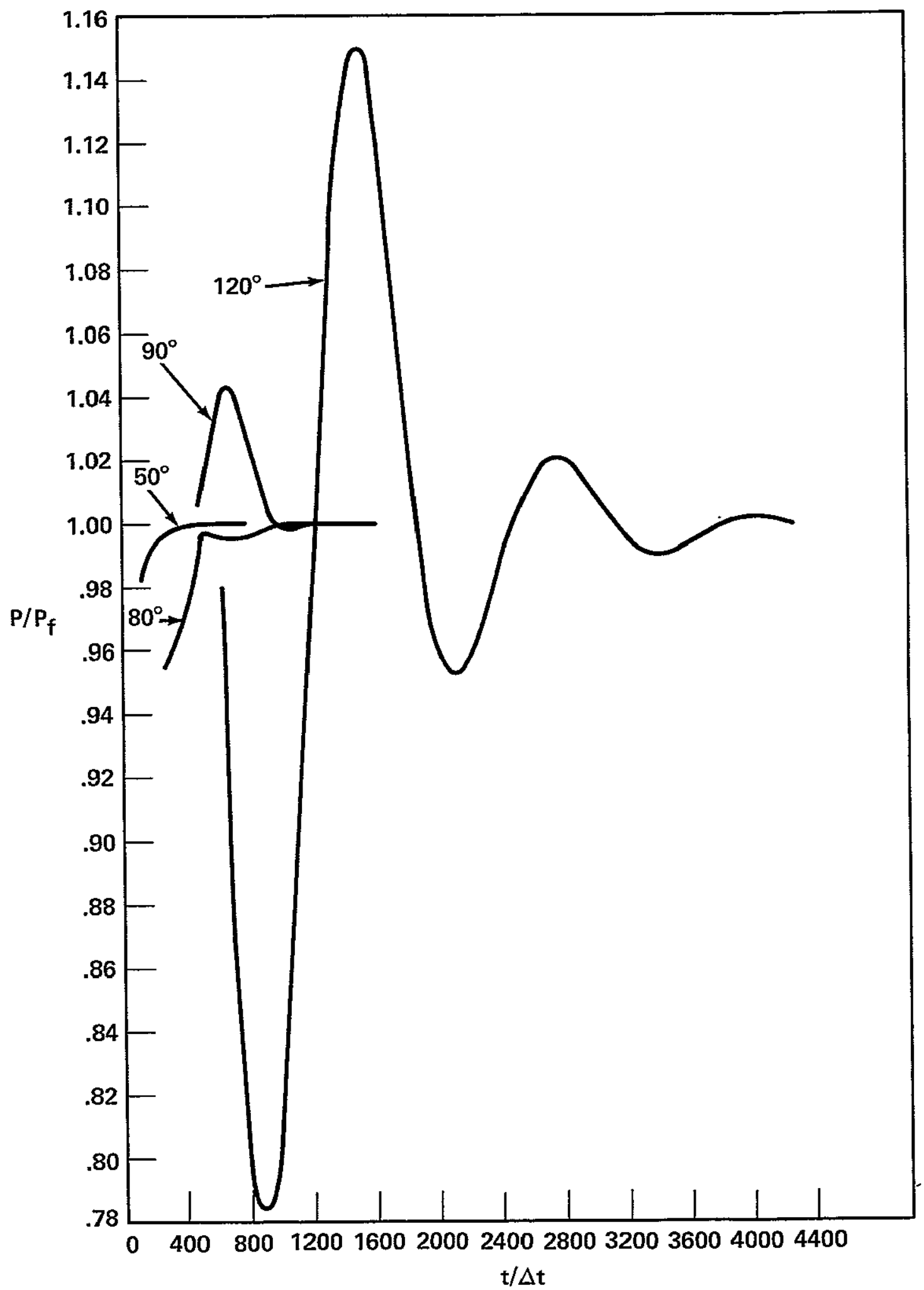

FIGURE 26 - PRESSURE TRANSIENTS NEAR VERTEX FOR DIFFERENT CONE ANGLES 


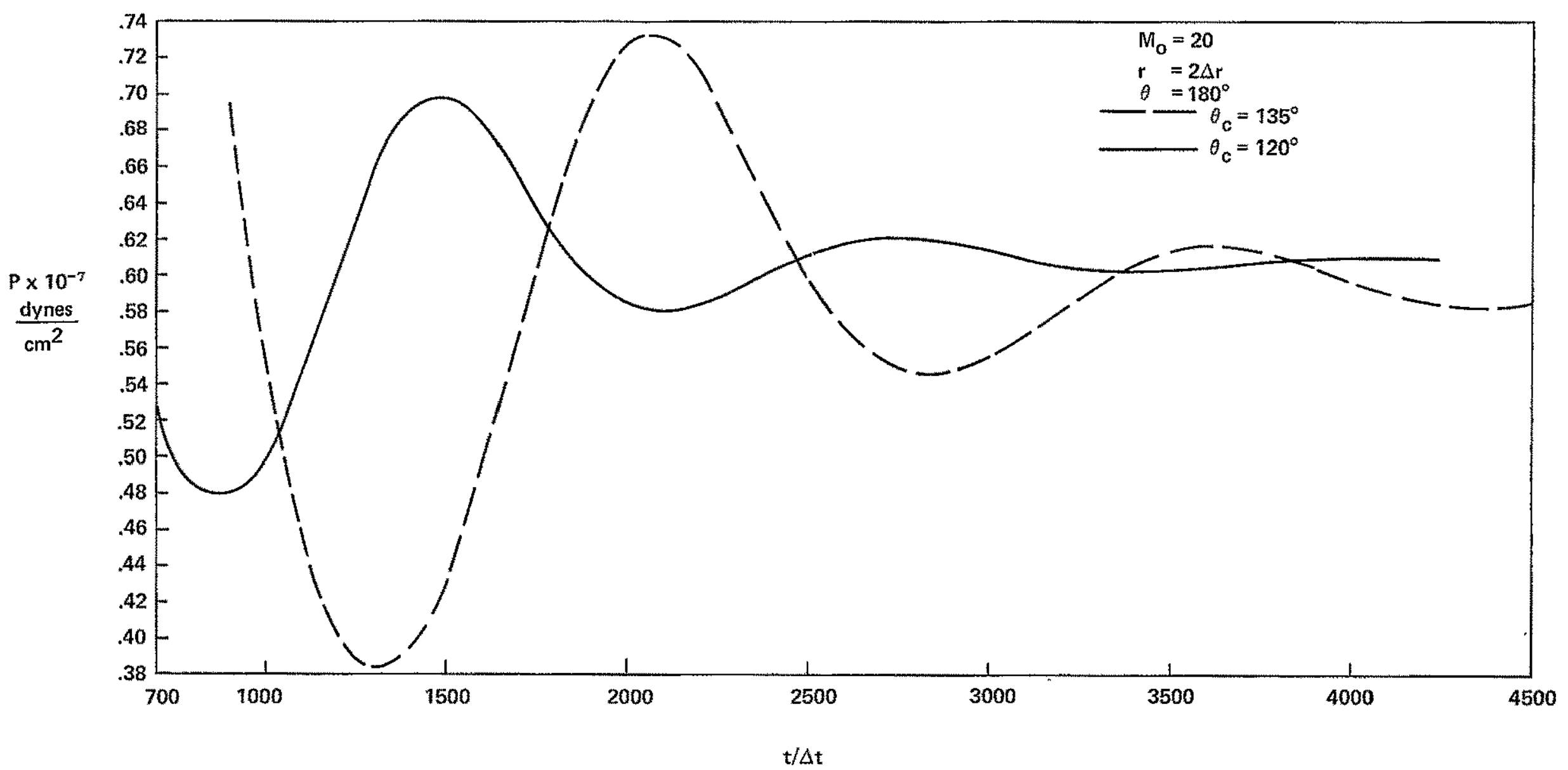

FIGURE 27 - PRESSURE TIME HISTORY FOR $120^{\circ}$ AND $135^{\circ}$ CONES 


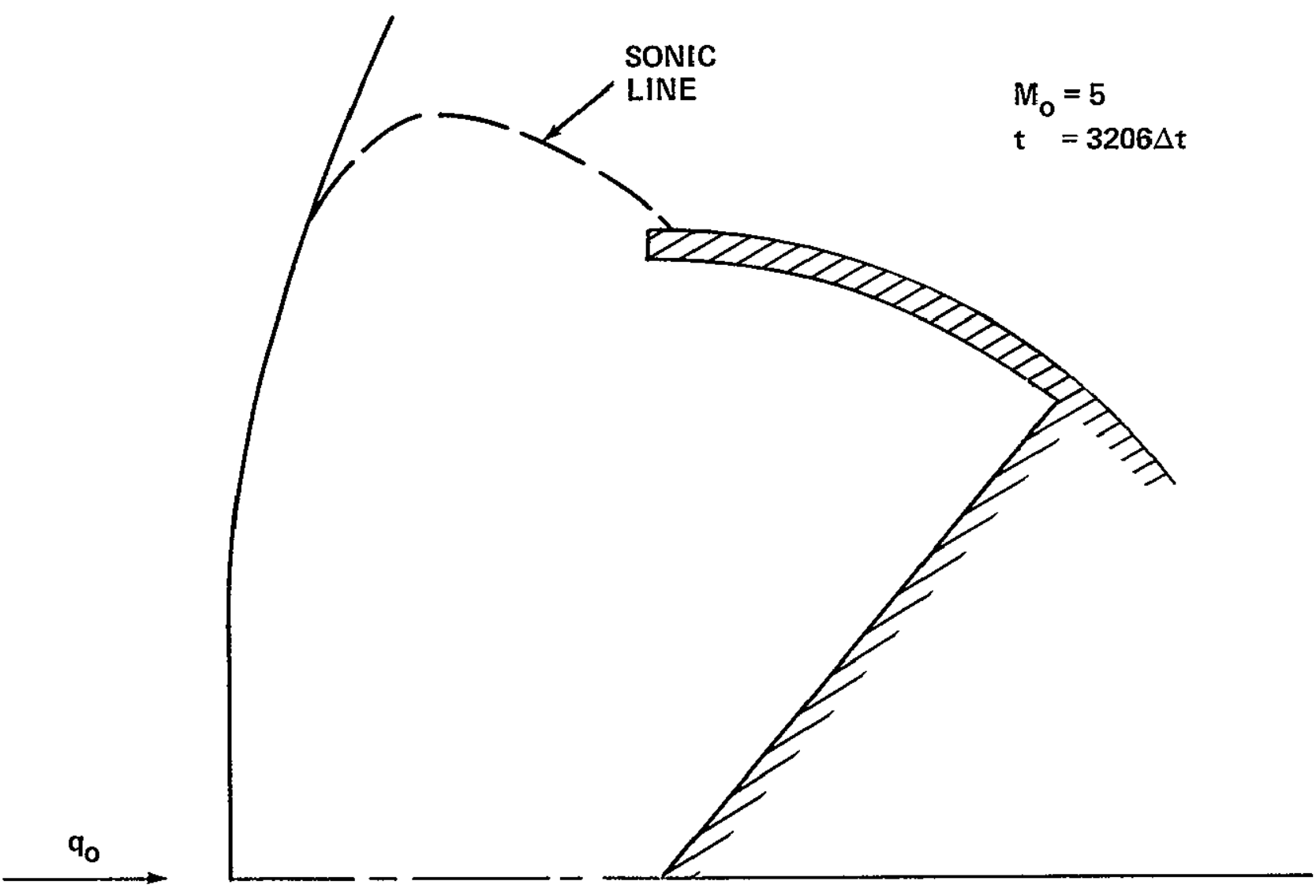

FIGURE 28 - SHOCK SHAPE FOR $50.56^{\circ}$ CONE WITH ROOF 


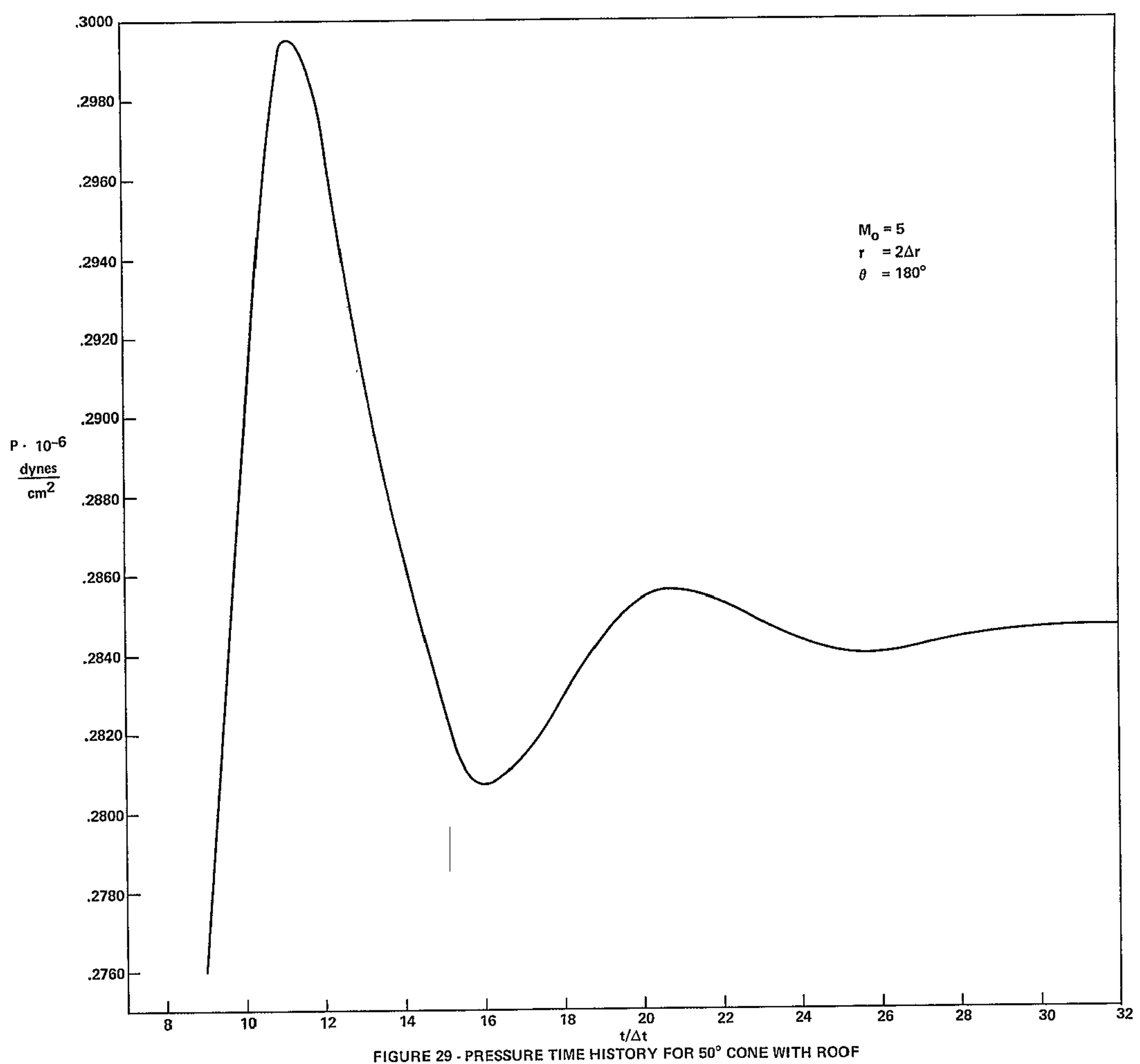




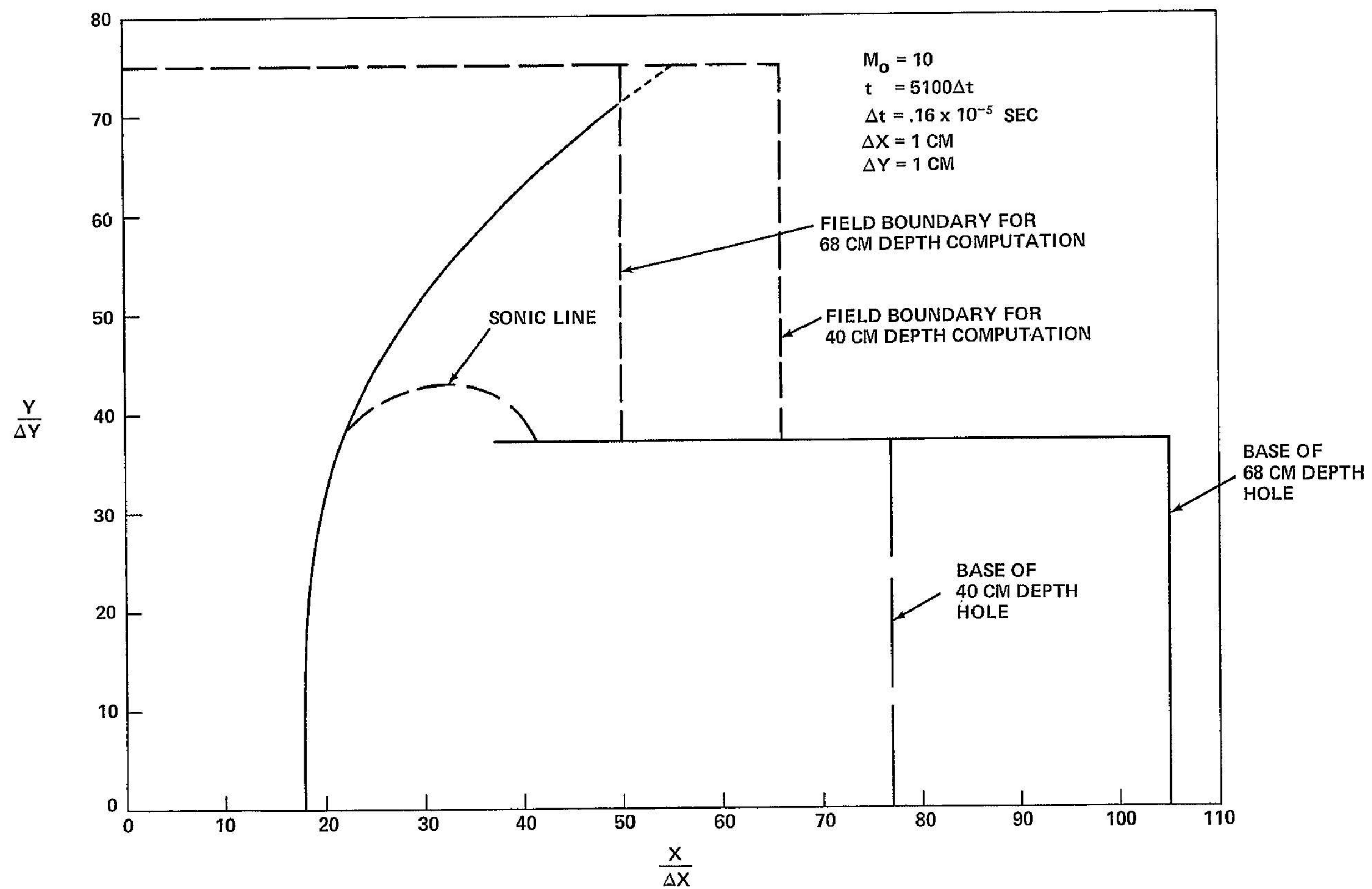

FIGURE 30 - SHOCK SHAPE AND SONIC LINE FOR $40 \mathrm{CM}$ AND 68 CM DEPTH CYLINDERS 


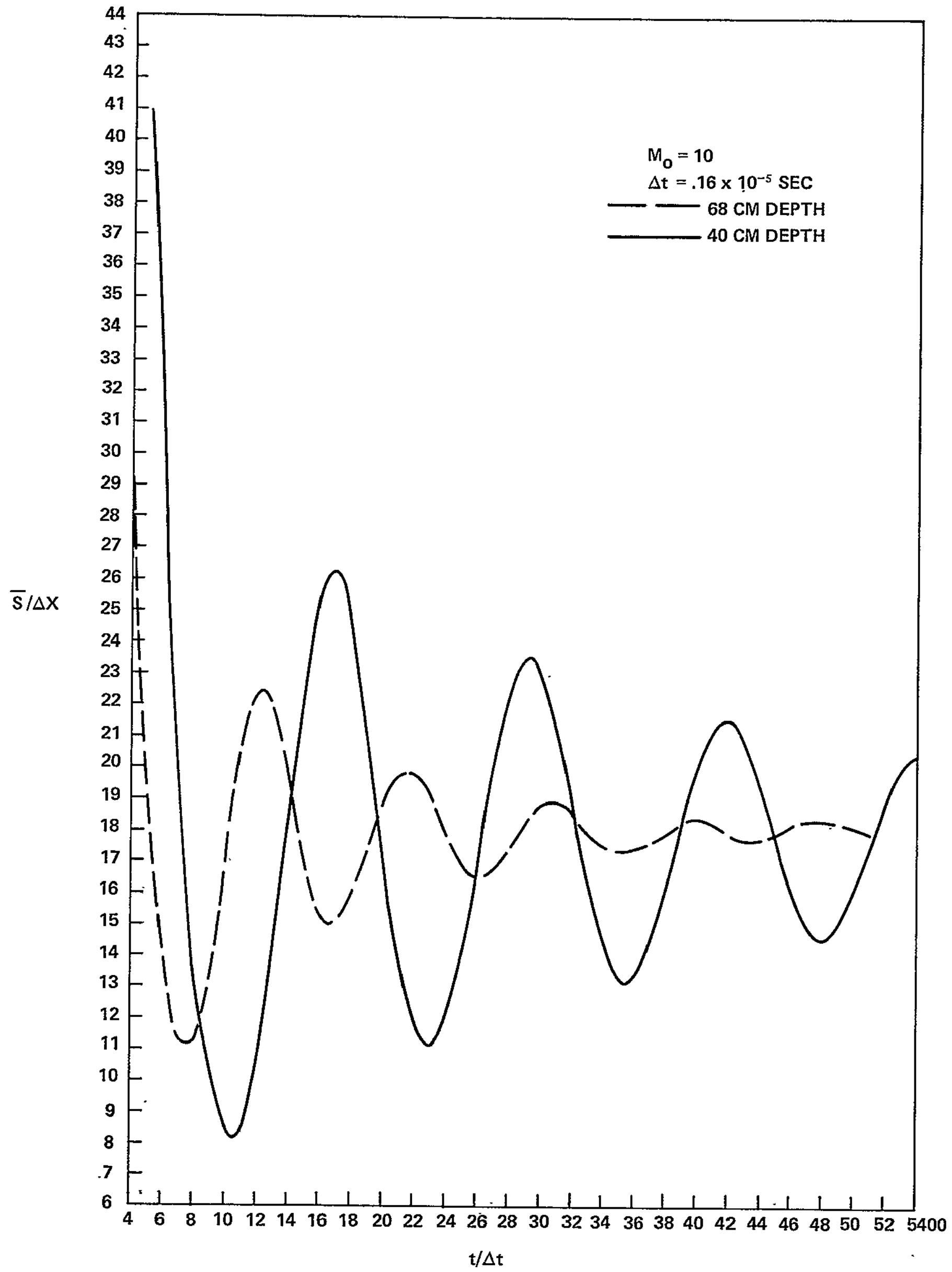

FIGURE 31 - SHOCK POSITION ON AXIS VS. TIME 


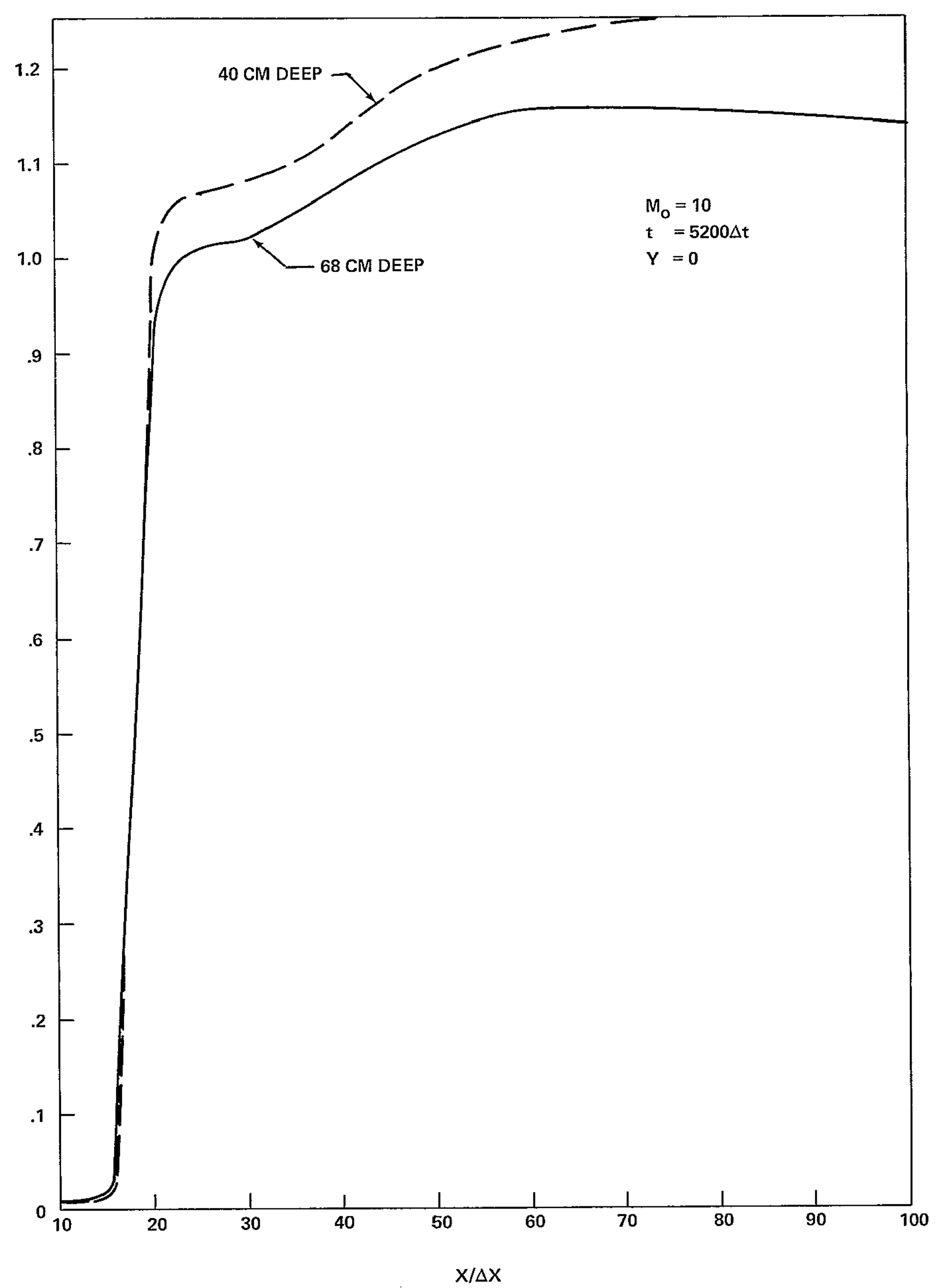

FIGURE 32 - PRESSURE PROFILE ALONG AXIS STREAMLINE 


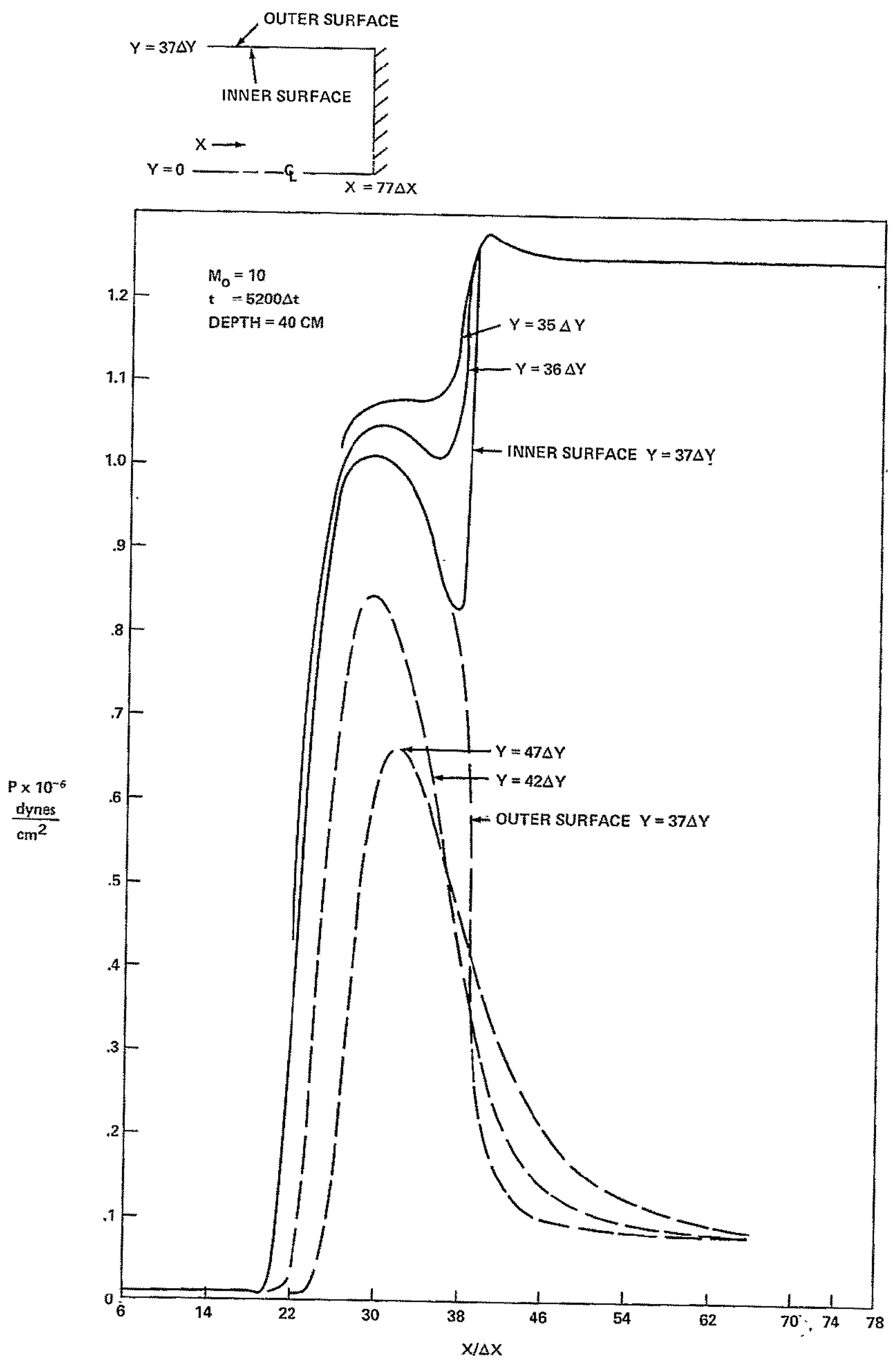

FIGURE 34 - PRESSURE IN AXIAL DIRECTION 
COMPLETE MEMORANDUM TO

CORRESPONDENCE FILES-

OFFICIAL FILE COPY

plus one white copy for each

additional case referenced

TECHNICAL LIERARY (4)

NASA $\cdot$ Headquarters

P. E. Culbertson/MT

R. F. Fellows/SL

E. W. Hall/MTG

W. Jakobowski/SL

A. S. Lyman/MR

M. A. Mitz/SI

J. B. Parkinson/RAA

I. R. Schwartz/RRF

A. O. Tischler/RS

\section{MSC}

D. M. Curry/ES5

G. B. Gibson/EE

D. H. Greenshields/Es5

B. Redd/EX2

R. C. Ried/ES5

G. Strouhal/ES5

\section{$\underline{\text { MSFC }}$}

R. J. Cochran/S\&E-COMP-R

W. K. Corcoran/PD-RV

\section{ARC}

A. E. Anderson/FAV

T. N. Canning/SVH

D. R. Chapman/ST

C. F. Coe/FAN

A. I. Erickson/SVS

D. J. Graham/FAN

C. F. Hansen/STF

I. Hunton/FAV

C. S. James/SVHF

E. D. Katzen/STH

M. W. Kelly/FSA

\section{COMPIETE MEMORANDUM TO}

H. K. Larson/STP

J. G. Marvin/STF

P. R. Nachtsheim/STP

W. A. Page/SVH

V. I. Peterson/STH

D. E. Reese, Jr./SV

I. Roberts/MAD

M. W. Rubesin/SVP

A. Seiff/SV

B. H. Wick/HFS

S. C. Sommers/SVHF

\section{GSFC}

R. Lehnert $/ 550$

F. O. Vonbun $/ 550$

$\underline{\text { IaRC }}$

N. D. Akey $/ 47.3$

R.'W. Barnwell/245

I. E. Beckwith/161

N. H. Bertram $/ 130$

R. E. Boughner/162

W. A. Brooks, Jr. $/ 206$

I. B. Callis, Jr./162

J. F. Campbel1/413

H. W. Carlson $/ 413$

N. I. Crabil1/159

W. F. Cuddihy/159

R. J. Duckett/159

A. Henderson, Jr./APD

D. T. Howe11/406

P. W. Huber $/ 160$

J. H. Eichtenstein/152C

F. E. Mershon/159

W. J. Nelson $/ 408$

J. F. Newcomb/159

S. I. Ocheltree/234

W. B. Olstad/162

C. B. Rumsey/214

J. C. South, Jr. $/ 245$

R. H. Sproul1/159

R. I. Stallings, Jr./413

J. R. Sterrett/164

G. M. Stokes $/ 206$

I. Taback/159 
J. D. Timmons/159

R. I. Trimpi/l6I

D. H. Tudor $/ 406$

BTL-Whippany

G. S. Deem

T. J. Kessler

R. E. Kiel

M. B. Lesser

I. Pelech

P. G. Simpkins

N. J. Zabusky

- Sandia Corporation
R. Eaton
R. Luna
J. Muir
K. Touryan
R. Woods

General Electric Corporation

R. Boericke

C. Jameson

C. Kyriss

S. Scala

Princeton University

R. Andres

S. Bogdonoff

S. Cheng

L. Crocco

W. Hayes

S. Lam

G. Mellor

Grumman Aircraft Corporation

G. Maise

University of Wichita

G. Zumwalt
Cornell'Aeronautical Iaboratory

M. Dunn

C. Treanor

Yale University

J. Anderson

J. Fenn

University of California at La Jolla

S. Iin

D. Miller

D. Olfe

S. Penner

C. Wang

Research Institute for Advanced Studies - Martin

S. Maslan

NYU - Courant Institute

S. Burstein

P. Iax

NYU - Department of Aeronautics and Astronautics

A. Ferri

R. Vaglio-Eaurin

V. Zakkay

Brooklyn Polytechnical Institute

G. Moretti

Boeing Scientific Research Laboratory

A. Goldburg

University of washington

A. Hertzberg 


\section{AVCO Corporation}

G. Sutton

R. Weiss

Naval Ordnance Laboratory

J. Anderson, Jr.

R. Feldhuhn.

D. Merritt

A. Winkelmann

Naval Research Laboratory

$$
\begin{aligned}
& \text { A. Cooper } \\
& \text { R. Shanny }
\end{aligned}
$$

Naval Ship Résearch and Development Center

F. Frenkiel

H. Lugt

R. Schwiderski

\section{Aerospace Corporation}

P. Crowell

R. Hartunian

H. Mirels

H. Sauerwein

TRW Industries

J. Chang

University of Delaware

F. Costello

Bellcomm, Inc.

G. M. Anderson

A. P. Boysen, Jx.

K. R. Carpenter

D. A. De Graaf

D. R. Hagner

N. W. Hinners

H. S. London
J. Z. Menard

J. M. Nervik

G. T. Orrok

W. Strack

J. I. Strand

W. B. Thompson

J. W. Timko

R. L. Wagner

M. P. Wilson

Center 102, Supervision

Department 1014, Supervision Department 1015, Supervision All Members, Department 1011 All Members, Department 1013 All Members, Department 2015 Central File

Department 1024 File COPY TO Library

Abstract Only

J. P. Downs

D. P. Ling 
()) 